\title{
Autômato Celular Probabilista, Modelos Unidimensionais de Trânsito e Teoria de Filas
}

\author{
Fredy Walther Castellares Cáceres
}

\author{
TESE APRESENTADA \\ AO \\ INSTITUTO DE MATEMÁTICA E ESTATÍSTICA \\ DA \\ UNIVERSIDADE DE SÃO PAULO \\ PARA \\ OBTENÇÃO DO GRAU DE DOUTOR \\ EM \\ ESTATÍSTICA \\ Área de Concentração: PROBABILIDADE
}

Orientador: Prof. Dr. Pablo Augusto Ferrari

$O$ autor agradece ao auxilio financeiro da FAPESP. Processo 00/04475-5

São Paulo, 9 de agosto de 2005 


\section{Autômato Celular Probabilista, Modelos Unidimensionais de Trânsito e Teoria de Filas}

Este exemplar corresponde à redação final da tese corrigida e defendida por Fredy Walther Castellares Cáceres

e aprovada pela comissão julgadora.

São Paulo, 9 de agosto de 2005.

Banca examinadora:

- Prof. Dr. Pablo Ferrari(Presidente) - IME-USP

- Prof. Dr. Vladimir Belitsky - IME-USP

- Prof. Dr. Claudio Landim - IMPA

- Prof. Dr. José Mário de Oliveira IF -USP

- Prof. Dr. Domingos Marchetti IF - USP 
Dedicado ao povo Brasileiro.

\section{Los Heraldos Negros}

Hay golpes en la vida, tan fuertes... Yo no sé!

Golpes como del odio de Dios; como si ante ellos,

la resaca de todo lo sufrido

se empozara en el alma... Yo no sé!

Son pocos; pero son... Abren zanjas oscuras

en el rostro más fiero y en el lomo más fuerte.

Serán tal vez los potros de bárbaros atilas;

o los heraldos negros que nos manda la Muerte.

Son las caídas hondas de los Cristos del alma

de alguna fe adorable que el Destino blasfema.

Esos golpes sangrientos son las crepitaciones

de algún pan que en la puerta del horno se nos quema.

$Y$ el hombre... Pobre... pobre! Vuelve los ojos, como

cuando por sobre el hombro nos llama una palmada;

vuelve los ojos locos, y todo lo vivido

se empoza, como charco de culpa, en la mirada.

Hay golpes en la vida, tan fuertes... Yo no sé!

\section{Masa}

Al fin de la batalla,

y muerto el combatiente, vino hacia él un hombre

y le dijo: «No mueras, te amo tanto!»

Pero el cadáver jay! siguió muriendo.

Se le acercaron dos y repitiéronle:

«No nos dejes! ;Valor! ; Vuelve a la vida!»

Pero el cadáver jay! siguió muriendo.

Acudieron a él veinte, cien, mil, quinientos mil, clamando: «Tanto amor, y no poder nada contra la muerte!»

Pero el cadáver jay! siguió muriendo.

Le rodearon millones de individuos, con un ruego común: «iQuédate hermano!»

Pero el cadáver jay! siguió muriendo.

Entonces, todos los hombres de la tierra

le rodearon; les vió el cadáver triste, emocionado;

incorporóse lentamente,

abrazó al primer hombre; echóse a andar

César Vallejo, poeta peruano 
Para mis amores Andréa y Luiza. 


\section{Agradecimentos}

Ao Povo Brasileiro representado pela FAPESP.

A Pablo por la orientación y sobre todo por la amistad.

A Eugene Pechersky por su disposición para discitir e enseñar.

A todos os amigos do instituto por fazerem o doutorado mais saboroso.....

Não poço me olvidar dos nossos funcionários da biblioteca.

A Andréa por dar-me a coisinha mais linda que temos, a nossa filha Luiza.

Finalmente, à família de Andréa por considerar-me mais um filho. 


\section{Resumo}

Modelos de trânsito de partículas aparecem na vida real e têm se convertido numa área de pesquisa muito ativa. Embora bastante estudados, desde 1992, com a publicação do artigo de Nagel-Schreckembrg ${ }^{1}$, por meio de simulações computacionais e por diversos métodos teóricos aproximados como os modelos de campo médio, existem poucos resultados rigorosos.

Mostramos resultados rigorosos para vários modelos de trânsito. Provamos a existência de transição de fase e propriedades assintóticas para o autômato celular 184 e para o modelo de Fukui-Ishibashi, que generaliza o autômato 184, permitindo movimento de partículas velozes. Introduzimos um autômato celular probabilista que resgata as propriedades dos modelos de Schadschneider-Schreckenberg, conhecidos como autômatos com regras slow-to-star. Provamos a existência de transição de fase, encontramos o fluxo assintótico. Introduzimos o autômato celular probabilista com distribuição inicial a medida produto de Bernoulli de densidade $\rho$ e a dinâmica de evolução dada por: Cada partícula espera um tempo aleatório que tem distribuição geométrica de parâmetro $p$ para mover-se pela primeira vez. Após este tempo, as partículas movem-se com velocidade 1 para sempre ou, em caso contrário, se deterão (várias partículas podem ocupar o mesmo sítio) se encontrarem alguma partícula parada na sua frente que bloqueie seu movimento. Neste caso as velocidades das partículas voltarão para 0 e as partículas ficarão bloqueadas até que a partícula ou as partículas que bloqueiam seus caminhos tenham partido. A partir deste instante, a partícula não bloqueada espera mais um tempo aleatório com distribuição geométrica para mover-se. Provamos que se $\rho<p$, a distância entre as partículas converge quase certamente à distância entre as saídas de um cliente numa fila estacionária Geom $/$ Geom $/ 1 / \infty$ associada ao sistema.

Finalmente, introduziremos um modelo de trânsito de partículas que é contínuo no tempo e no espaço, que denominaremos Modelo Pontual. O processo pontual distribui as partículas na reta $\mathbb{R}$ segundo um processo de Poisson marcado de parâmetro $\lambda$. As marcas são os tempos de espera para que as partículas mudem de velocidade 0 para velocidade 1. Cada partícula espera um tempo aleatório que tem distribuição exponencial de média 1 para mover-se pela primeira vez. Após este tempo, as partículas movem-se com velocidade 1 para sempre ou, em caso contrário, se deterão se encontrarem alguma partícula parada na sua frente que bloqueie seu movimento. Neste caso, as velocidades das partículas voltarão para 0 e as partículas ficarão bloqueadas até que a partícula ou as partículas que bloqueiam seus caminhos tenham partido. A partir deste instante, a partícula não bloqueada espera mais um tempo aleatório com distribuição exponencial para mover-se. Em todo instante de tempo teremos partículas que se movem a velocidade 1 ou bloqueadas. Provamos que se $\lambda<1$, a distância entre as partículas converge quase certamente à distância entre as saídas de um cliente numa fila estacionária $M / M / 1 / \infty$ associada ao sistema.

\footnotetext{
${ }^{1}$ Primeiro modelo de trânsito unidimensional estudado como autômato celular probabilista
} 


\section{Resumo}

Particle traffic models appear in real life and have turned into a very active area of research. Although well studied since 1992 with the publication of the Nagel-Schreckembrg article $^{2}$, by means of computer simulations and by several approximate theoretical methods such as mean field models, there exist not a lot rigorous results.

We give rigorous results for several traffic models. We prove the existence of phase transition and asymptotic properties for cellular automata 184 and for the Fukui-Ishibashi model, which generalizes the 184 automata, permitting movement of fast particles. We introduce a probabilistic cellular automata which retains the properties of the SchadschneiderSchreckenberg models, known as automata with slow-to-star rules. We prove the existence of phase transition and asymtoptic current. We introduce the probabilistic cellular automata whose initial average distribution is a Bernoulli product of density $\rho$ and whose evolution dynamics are given by: Every particle waits a random time which has a geometric distribution with parameter $p$ to move for the first time. After this time, the particles move with velocity 1 forever or, in the opposite case, will be stopped (several particles can occupy the same site) if they meet a particle blocking their way. In this case the velocities return to 0 and remain blocked until the particle or particles blocking the way have left. From this moment the unblocked particle waits a further random time with a geometric distribution before moving. We prove that if $\rho<p$ the distance between particles converge almost surely to the distance between the exits of a client in a the stationary discrete queue Geom/Geom $/ 1 / \infty$ associate to the systems.

Finally we introduce a particle traffic model which is continuous in space and time, which we denominate the Point Traffic Model. The point process distributes the particles on a straight line $\mathbb{R}$ following a Poisson process of parameter $\lambda$ and the marks are the waiting time for the particles to change from velocity 0 to velocity 1 . Each particle waits a random time which has an exponential distribution of average 1 to move for the first time. After this time, the particles move with velocity 1 forever or, in the opposite case, will be stopped if they meet a particle blocking their way. In this case the velocities return to 0 and remain blocked until the particle or particles blocking the way have left. From this moment the unblocked particle waits a further random time with an exponential distribution before moving. At every instant of time we will have particles which move the blocked particles to velocity 1 . We prove that if $\lambda<1$ the distance between particles converge almost surely to the distance between the exits of a client in a stationary queue $M / M / 1 / \infty$ associate to the systems.

\footnotetext{
${ }^{2}$ The first model of unidimensional traffic studied through probabilistic cellular automata.
} 


\section{Sumário}

1 Introdução 3

2 Autômato Celular $184 \quad 6$

2.1 Definição do modelo . . . . . . . . . . . . . . . . . . . . 7

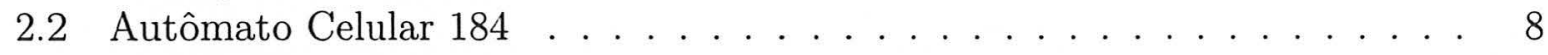

2.3 Definições e Notação . . . . . . . . . . . . . . . . . . . . . . . . . . . . 9

2.3.1 Propriedades do operador do autômato celular 184 atuando em con-

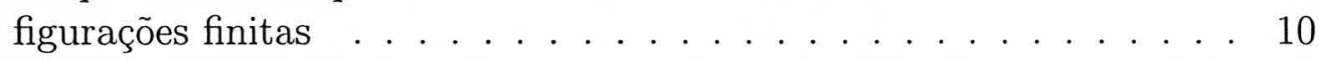

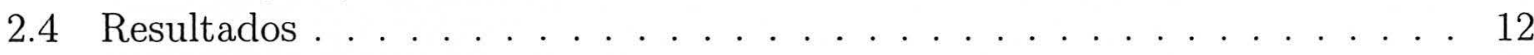

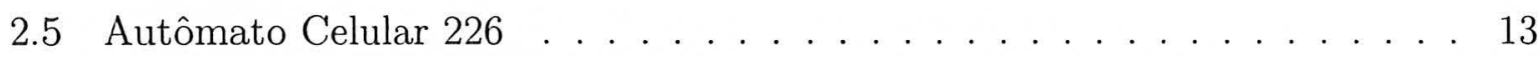

2.6 Processo de Aniquilação Balística . . . . . . . . . . . . . . . . . . . . . . 13

2.7 Modelo de Crescimento de Superfícies . . . . . . . . . . . . . . . . . . . 14

2.8 Prova dos Teoremas . . . . . . . . . . . . . . . . . . . . . . . . . . . . . . . . . .

2.9 Estimativa do fluxo do AC 184. . . . . . . . . . . . . . . . . . 24

3 Modelo de Fukui e Ishibashi 27

3.1 Definição do Modelo . . . . . . . . . . . . . . . . . . 27

3.2 Generalização do Modelo de Crescimento de Superfícies . . . . . . . . . . . 28

3.3 Resultados . . . . . . . . . . . . . . . . . . 29

4 Autômato Celular com Aceleração Aleatória 34

4.1 Definição do modelo . . . . . . . . . . . . . . . . . . . . . . . 34

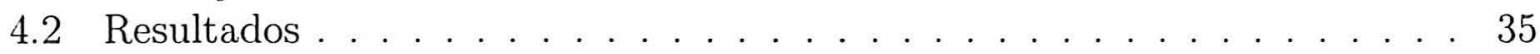

4.3 Modelo Modificado do ACAA . . . . . . . . . . . . . . . . . . . . . . . . . . . . . . . . . . . . . . . .

4.4 Relação do ACAA com um Sistema de Filas Discreto . . . . . . . . . . . 39

5 Modelo Pontual de Trânsito em $\mathbb{R} \quad 40$

5.1 Definição do modelo . . . . . . . . . . . . . . . . . . . . . . 41

5.1 .1 Construção do Processo em $\mathbb{R}$. . . . . . . . . . . . . . . . . . 44

5.2 Resultados . . . . . . . . . . . . . . . . . . . . . . 44

5.3 Construção Gráfica do Modelo Pontual . . . . . . . . . . . . . . . . . 44

5.3.1 Construção das trajetórias $\Pi($.$) condicionada a C_{0}$ livre desde o início. 44

5.3.2 Cálculo da trajetória da partícula $C_{n+1}$ a partir da trajetória da partícula $C_{n} \ldots \ldots \ldots \ldots \ldots$. . . . . . . . . . 48 
5.3.3 A trajetórias $\Pi($.$) como função do processo \mathbb{M}$ condicionada a $C_{0}$ livre desde o início . . . . . . . . . . . . . . . . . . 51

5.4 Fila $M / M / 1 / \infty$ FIFO . . . . . . . . . . . . . . . . . . 55

5.4 .1 Construção de um ciclo . . . . . . . . . . . . . . . . 56

5.4 .2 Teoremas e Relações . . . . . . . . . . . . . . . . . . . . . . 57

5.5 Modelo de Filas Associado ao Modelo Pontual . . . . . . . . . . . . . . . . 58

5.5 .1 Teoremas e Relações . . . . . . . . . . . . . . . . . . . 6 60 60

5.6 Prova dos Resultados . . . . . . . . . . . . . . . . . . . 66 


\section{Capítulo 1}

\section{Introdução}

Modelos de trânsito unidimensionais são sistemas de infinitas partículas distribuídas nos números inteiros, $\mathbb{Z}$, ou na reta real, $\mathbb{R}$, isto é, o espaço pode ser discreto ou contínuo. Associamos ao sistema uma dinâmica de evolução que pode ser determinística ou probabilista. A evolução pode ser em tempo discreto ou contínuo. Em cada instante de tempo todas as partículas atualizam suas posições simultaneamente. Se o sistema é discreto espacial e temporalmente, então, o modelo de trânsito é um autômato celular. Se a dinâmica é probabilista, teremos um autômato celular probabilista. Se o sistema é contínuo espacialmente e temporalmente, o chamaremos de modelo pontual de trânsito.

O interesse nos modelos de trânsito é muito antigo. Este começou em 1935 quando Greenshield ${ }^{1}$ realizou o primeiro estudo sobre trânsito. Em 1950, já existia uma grande quantidade de publicações em revistas de engenharia e de pesquisa operacional. Estes trabalhos introduziram o diagrama fundamental mostrando a relação entre fluxo de partículas e a densidade. Encontraram a existência de diversas classes de fluxo de trânsito, problema que ainda hoje é relevante. A motivação para tais estudos são auto-evidentes. Como Greenberg [17] escreveu em 1959, "O volume de trânsito veicular tem ultrapassado a capacidade das estradas e ruas de todas as grandes nações. Existe uma crescente necessidade de entender a dinâmica do trânsito e obter uma descrição matemática deste fenômeno."

Embora a dinâmica do trânsito tenha sido muito estudada, não existiam, até uns poucos anos atrás, resultados rigorosos que explicassem a riqueza destes fenômenos. Um dos primeiros resultados analíticos para modelos determinísticos foi apresentado por Fuks[14] em 1998, que calculou exatamente o fluxo do modelo de Fukui e Ishibashi usando técnicas combinatórias. O modelo de Nagel-Schrekenberg e os modelos de slow-to-star com $v_{\max }>1$ estão em aberto. O Modelo de Nagel-Schreckenberg com $v_{\max }=1$ foi resolvido rigorosamente por Yaguchi[30], em 1986. Aparentemente, Nagel e Schrenkenberg não souberam disto, porque a equivalência entre os dois modelos não é imediata. Yaguchi estudou o processo totalmente assimétrico em tempo discreto com atualização em paralelo. Ele obteve as medidas estacionárias usando técnicas de acoplamento criadas por Liggett[20] e outros. Belitsky e Ferrari[6](publicado em 2005) encontraram as medi-

\footnotetext{
${ }^{1}$ Ver Helbing $[18]$ e Chowdhury et al [11] para uma revisão dos modelos de trânsito.
} 
das invariantes para o autômato celular 184, usando um outro processo conhecido como processo de aniquilação balística. Belitsky et al[7] estudaram o modelo trânsito em duas vias (câmbio de via é permitido) usando o autômato celular 184. Blank muito recentemente, 2003-2004, estudo modelos determinísticos usando técnicas de sistemas dinâmicos ver [8],[9]. Angel, Holroyd e James[1] acabam de publicar (março de 2005) o primeiro resultado rigoroso do modelo de Biham-Middleton-Levine. Este modelo estuda trânsito em duas dimensões.

Nesta tese apresentamos resultados rigorosos para vários modelos de trânsito. A organização da tese é a seguinte: No capítulo dois estudaremos o autômato celular determinístico classificado por Wolfram como o autômato 184. Introduziremos outros dois processos intimamente relacionados com este autômato. Estes processos são o processo de aniquilação balística e o processo de crescimento de superfícies. Com ajuda destes processos provamos a existência de transição de fase e propriedades assintóticas do fluxo e velocidade das partículas.

No capítulo três estudaremos o modelo determinístico de Fukui-Ishibashi. Este modelo generaliza o autômato celular 184, permitindo movimento de partículas velozes. Para este modelo estendemos as técnicas usadas no autômato celular 184 para provar também a existência de transição de fase e propriedades assintóticas do fluxo e velocidade das partículas.

No capítulo quatro introduzimos um autômato celular probabilista, que denominamos de autômato celular com aceleração aleatória, que resgata as propriedades dos modelos de Schadschneider-Schreckenberg, conhecidos como autômatos com regras slow-to-star. Provamos a existência de transição de fase e calculamos o fluxo assintótico. Neste modelo o tipo de interação entre as partículas é de exclusão.

Introduzimos uma variante do autômato com aceleração probabilista. Nesta variante enfraquecemos a interação tipo exclusão permitindo que várias partículas ocupem o mesmo sítio. O autômato celular probabilista tem distribuição inicial a medida produto de Bernoulli de densidade $\rho$ e a dinâmica de evolução dada por: Cada partícula espera um tempo aleatório que tem distribuição geométrica de parâmetro $p$ para mover-se pela primeira vez. Após este tempo, as partículas movem-se com velocidade 1 para sempre ou, em caso contrário, se deterão (várias partículas podem ocupar o mesmo sítio) se encontrarem alguma partícula parada na sua frente que bloqueie seu movimento. Neste caso as velocidades voltarão para 0 e ficarão bloqueadas até que a partícula ou as partículas que bloqueiam seus caminhos tenham partido. A partir deste instante, a partícula não bloqueada espera mais um tempo aleatório com distribuição geométrica para mover-se. Apresentamos uma construção gráfica que permite mapear o modelo pontual em um sistema de filas discreto com um único servidor. Provamos que se $\rho<p$, a distância entre as partículas converge é quase certamente à distância entre as saídas de um cliente numa fila discreta estacionária Geom $/$ Geom $/ 1 / \infty$ associada ao sistema.

Finalmente, no capítulo cinco introduzimos um modelo de trânsito de partículas que é contínuo no tempo e no espaço, que denominaremos Modelo Pontual. O processo pontual distribui as partículas na reta $\mathbb{R}$ segundo um processo de Poisson de parâmetro $\lambda$ e as marcas são os tempos de espera para que as partículas mudem de velocidade 0 para 
velocidade 1. Cada partícula espera um tempo aleatório que tem distribuição exponencial de média 1 para mover-se pela primeira vez. Após este tempo, as partículas movem-se com velocidade 1 para sempre ou, em caso contrário, se deterão se encontrarem alguma partícula parada na sua frente que bloqueie seu movimento. Neste caso, as velocidades voltarão para 0 e ficarão bloqueadas até que a partícula ou as partículas que bloqueiam seus caminhos tenham partido. A partir deste instante, as partículas não bloqueadas esperam mais um tempo aleatório com distribuição exponencial para mover-se. Em todo instante de tempo teremos partículas que se movem a velocidade 1 ou bloqueadas. Apresentamos uma construção gráfica que permite mapear o modelo pontual em um sistema de filas com um único servidor. Esta construção é feita encontrando primeiro as trajetórias das partículas. Encontramos que a trajetória de uma partícula qualquer depende só da trajetória da partícula que está na sua frente. Provamos que se $\lambda<1$ a família de trajetórias tem um processo pontual marcado associado. Este processo está formado pelas posições das partícula e pelo último intervalo de tempo de espera para que as partículas fiquem livres. Com isto provamos que quando $\lambda<1$ a distância entre as partículas converge quase certamente à distância entre as saídas de um cliente numa fila estacionária $M / M / 1 / \infty$ associada ao sistema.

Finalmente, para facilitar a leitura faremos alguns comentários. No iníco de cada capítulo introduzimos as definições e os elementos básicos do modelo correspondente, logo, imediatamente enunciamos os resultados principais do capítulo, para finalmente provar os teoremas no final de cada capítulo. 


\section{Capítulo 2}

\section{Autômato Celular 184}

Autômatos celulares, denotados por AC, são sistemas dinâmicos discretos no espaço e no tempo. Em geral, eles podem ser vistos como pontos em um reticulado regular $(\mathbb{Z}$, no caso unidimensional) e atualizados em paralelo de acordo a regras de interação local, onde o conjunto de valores de cada ponto é um conjunto finito. O primeiro modelo de trânsito usando autômatos celulares com dinâmica probabilistas foi proposto por Nagel-Schrekenberg, ${ }^{1}$ em 1992. Muitos aspectos teóricos deste modelo ainda não são compreendidos e têm sido propostos modelos simplificados, incluindo modelos de autômatos celulares determinísticos. O limite determinístico do modelo de Nagel-Schreckenberg com velocidade máxima 1 é o autômato celular 184.

O Autômato Celular 184, denotado por AC 184, foi introduzido por Wolfram[31] que deu para ele o número de 184 na sua classificação ${ }^{2}$. Uma vez distribuídas as partículas nos inteiros $\mathbb{Z}$, no máximo uma partícula por sítio, sua regra pode ser descritas pela frase: "em cada instante todas as partículas que têm o sítio vazio à sua direita pulam no próximo instante uma unidade à direita".

Neste capítulo apresentaremos nossos resultados para o AC 184. Usaremos para as provas dois processos intimamente relacionados. O processo de aniquilação balística (denotado PAB) e o modelo de crescimento de superfícies (denotado MCS). Essas relações foram estabelecidas por Krug e Spohn [19]. Elkens e Frish [12] estudaram o processo de aniquilação balística, conhecido pelos físicos, como o modelo de reação e aniquilação $A+B \rightarrow$ inerte. Este autômato é definido pelas seguintes regras: No instante $t=0$ colocam-se partículas em cada ponto de $\mathbb{Z}$, no máximo uma partícula por sítio. Cada partícula tem velocidade +1 e -1 com probabilidade $1 / 2$, independente das outras. As partículas com velocidade +1 vão para direita e as de velocidade -1 para esquerda; partículas que se encontram aniquilam-se e desaparecem do sistema para sempre.

Belitsky e Ferrari [5] calcularam a distribuição desse autômato em cada instante $t \in \mathbb{N}$. Em um artigo posterior [6] encontraram as medidas invariantes e provaram a existência de medidas de choque para o autômato celular 184 .

Nós daremos condições necessárias e suficientes para que uma partícula entre em estado

\footnotetext{
${ }^{1}$ Ver o artigo original[22]

${ }^{2}$ Veremos na próxima seção a fórmula que classifica os autômatos celulares
} 
de fluxo livre e para a existência de transição de fase. Provaremos que $1 / 2$ é o ponto crítico do modelo e encontraremos propriedades assintóticas do fluxo e velocidade das partículas.

\subsection{Definição do modelo}

Seja $\mathcal{Q}$ um conjunto contável de cardinalidade $q$, que será chamado de alfabeto. Se $q$ é finito, assumiremos $\mathcal{Q}=\{0,1,2, \ldots, q-1\}$. Uma biseqüência (configuração) sobre $\mathcal{Q}$ é uma função de $\mathbb{Z}$ em $\mathcal{Q}$. Denotemos por $\Omega, \Omega=\mathcal{Q}^{\mathbb{Z}}$, o espaço de configurações (conjunto de todas as biseqüências sobre $\mathcal{Q}$ ). Se $\eta \in \Omega$, será chamado de configuração e $\eta(i), \forall i \in \mathbb{Z}$, será o valor da configuração $\eta$ no ponto $i$.

Como o autômato evolui no tempo, $\left\{\eta_{t}\right\}_{t \in \mathbb{N}}$, indica o autômato em todos os tempos e $\eta_{t}$ será a configuração do autômato no tempo $t$. Então, $\eta(i, t)$ será o valor do autômato no ponto $i$ no instante $t$.

Seja $s: \mathbb{Z} \times \mathbb{N} \longmapsto \mathcal{Q}$ que satisfaz a equação

$$
s(i, t+1)=f\left(s\left(i-r_{l}, t\right), s\left(i-r_{l-1}, t\right), \ldots, s\left(i+r_{r}, t\right)\right)
$$

para todo $i \in \mathbb{Z}$ e todo $t \in \mathbb{N}$. $\mathcal{Q}$ é o conjunto finito de estados igual a $\{0,1,2, \ldots q-1\}$. $s(i, t)$ representa o estado do sítio $i$ no tempo $t$ e a função $f: \mathcal{Q}^{r_{l}+r_{r}+1} \longmapsto \mathcal{Q}$ é a regra de evolução do AC. Os números inteiros positivos $r_{l}$ e $r_{r}$ são, respectivamente, os rádios à esquerda e à direita do ponto $i$. A partir de agora, $f$ será dita uma função local ou regra de $n$ entradas, onde $n$ é o número $\left(r_{l}+r_{r}+1\right)$ de argumentos de $f$.

Definimos a função global $F: \Omega \longmapsto \Omega$ tal que

$$
\left[F\left(\eta_{t+1}\right)\right](i)=f\left(\eta\left(i-r_{l}, t\right), \eta\left(i-r_{l-1}, t\right), \ldots, \eta\left(i+r_{r}, t\right)\right)
$$

para toda configuração $\eta_{t}$.

Segundo Wolfram[31], a cada regra $f$ corresponde seu número de regra $N(f)$ definido por

$$
N(f)=\sum_{\left(x_{1}, x_{2}, \ldots, x_{n}\right) \in \mathcal{Q}^{n}} f\left(x_{1}, x_{2}, \ldots, x_{n}\right) q^{q^{n-1} x_{1}+q^{n-2} x_{2}+\ldots .+q^{0} x_{n}}
$$

Exemplo 1: Autômato 184. Tomemos $\mathcal{Q}=\{0,1\}$ e definamos as regras do autômato 184 mediante a função com 3 entradas $f$ como

$$
f\left(x_{1}, x_{2}, x_{3}\right)=x_{2}+\min \left\{x_{1}, 1-x_{2}\right\}-\min \left\{x_{2}, 1-x_{3}\right\}
$$

Portanto, dado um ponto, a regra depende só de seu primeiro vizinho à esquerda e à direita. A tabela de seus valores são:

$$
\begin{aligned}
& f(0,0,0)=0, f(0,0,1)=0, f(0,1,0)=1, f(0,1,1)=1 \\
& f(1,0,0)=1, f(1,0,1)=1, f(1,1,0)=0, f(1,1,1)=1
\end{aligned}
$$


Vemos que os uns podem ser interpretados como "carros" e os zeros como "vazios". Carros se movem à direita. Se "carros"tem um espaço vazio na sua frente, então, se moveram uma unidade para direita no próximo instante.

Calculando o número segundo a fórmula de Wolfram

$$
N(f)=\sum_{\left(x_{1}, x_{2}, x_{3}\right)=0}^{1} f\left(x_{1}, x_{2}, x_{3}\right) 2^{2^{3-1} x_{1}+2^{3-2} x_{2}+2^{0} x_{3}}
$$

encontramos que o número correspondente a $f$ é 184 .

Exemplo 2: Autômato 226. Tomemos $\mathcal{Q}=\{0,1\}$ e definamos as regras do autômato $f_{226}$ como

$$
f_{226}\left(x_{1}, x_{2}, x_{3}\right)=x_{2}+\min \left\{x_{3}, 1-x_{2}\right\}-\min \left\{x_{2}, 1-x_{1}\right\}
$$

Depois de simples álgebra encontramos que

$$
f_{226}\left(x_{1}, x_{2}, x_{3}\right)=1-f_{184}\left(1-x_{1}, 1-x_{2}, 1-x_{3}\right)
$$

mostrando que estas duas regras não são fundamentalmente distintas. Na verdade, se o AC 184 modela partículas viajando à direita, o AC 226 modelará buracos viajando à direita. Esta propriedade do $\mathrm{AC} 226$ será dita de dual e, portanto, o AC 226 será o dual do AC 184. A propriedade de dualidade é muito importante, porque devido à simetria partícula/buraco, estudar a dinâmica do modelo AC 184 com alta densidade será equivalente a estudar o AC 226 com baixa densidade de buracos.

Autômatos 184 e 226 são exemplos simples não triviais de autômatos celulares conservativos.

\subsection{Autômato Celular 184}

Como estamos interessados no modelo de trânsito na reta infinita $\mathbb{Z}$, definamos o autômato celular 184 pela sua função global. O AC 184 tem dois estados 0,1 e a seguinte regra de evolução: Ao tempo $t=0$ partículas são distribuídas nos sítios de $\mathbb{Z}$, ao mais uma partícula por sítio. Representamos um sítio ocupado por uma partícula por 1 e vazio por 0 . O autômato começará com a medida produto de Bernoulli $\nu_{\rho}$ de densidade $\rho$, o qual distribui uma partícula em cada sítio independentemente com densidade $\rho \in[0,1]$.

A dinâmica do $\mathrm{AC} 184$ será definida pelo operador $F:\{0,1\}^{\mathbb{Z}} \rightarrow\{0,1\}^{\mathbb{Z}}$ tal que $\eta_{t+1}=F \eta_{t}$. Se $\eta_{t} \in\{0,1\}^{\mathbb{Z}}$ representa a configuração do autômato em um arbitrário instante de tempo $t, t \in \mathbb{N}=\{0,1,2 \ldots\}$, então, $\eta_{t+1} \in\{0,1\}^{\mathbb{Z}}$ é definido por

$$
\eta_{t+1}(i)=F \eta_{t}(i):= \begin{cases}1 & \text { se } \eta(i+1)=\eta(i)=1 \\ 1 & \text { se } \eta(i)=1-\eta(i-1)=0 \\ 0 & \text { caso contrário }\end{cases}
$$

para todo $i \in \mathbb{Z}$. 
Formalmente $\mathrm{AC} 184$ é uma seqüência de variáveis aleatórias $\left\{\eta_{t}, t \in \mathbb{N}\right\}$ tal que $\eta_{t+1}=$ $F \eta_{t}$ para todo $t$.

Consideremos para o AC 184 o espaço de probabilidade $\left(\{0,1\}^{\mathbb{Z}}, \mathcal{B}, \nu_{\rho}\right)$, onde $\mathcal{B}$ é a sigma álgebra gerada pelos conjuntos cilíndricos de $\{0,1\}^{\mathbb{Z}}$ e $\nu_{\rho}$ é a medida produto de Bernoulli que colocamos no início do processo. Notemos que o AC 184 tem dinâmica determinística e modela o movimento de partículas idênticas para a direita na reta infinita $\mathbb{Z}$, onde a aleatoriedade do modelo está introduzida pela condição inicial.

O AC 184 tem duas contra partes estocásticas: Uma é o processo de exclusão simples totalmente assimétrico (denotado TASEP), onde a atualização das posições das partículas é aleatória; a segunda tem a mesma evolução que um autômato 184, com a diferença que as partículas que podem pular pulam com probabilidade $p$ independentemente das demais partículas. Este processo é exatamente o modelo de Yaguchi[30] que se pode provar que é equivalente ao modelo Nagel-Schreckenberg com velocidade máxima permitida igual a 1. As medidas invariantes para TASEP e para o modelo de Yaguchi foram encontradas por Liggett[20] e Yaguchi respectivamente.

\subsection{Definições e Notação}

Definição 1. Para o autômato celular 184

- Denotemos por $C_{n}$ o índice da $n$-ésima partícula e por $x_{n}$ sua posição.

- Definimos por $1_{n}:=\underbrace{11 \ldots 11}_{n \text { uns }}$ e $0_{n}:=\underbrace{00 \ldots 00}_{n \text { zeros }}$

- Dada uma configuração inicial de partículas $\eta_{0}$. Dizemos que a partícula $C_{n}$ está em estado de fluxo livre se existe um tempo aleatório $t_{n}\left(\eta_{0}\right)$ que depende da configuração inicial, tal que a partícula avança à velocidade 1 para todos os instantes de tempo maiores ou iguais a $t_{n}\left(\eta_{0}\right)$.

- Dada uma configuração inicial de partículas $\eta_{0}$. Dizemos que a partícula $C_{n}$ está bloqueada na posição $x_{n}$ se existe uma partícula com velocidade zero no ponto $x_{n}+1$.

- Dada uma configuração inicial de partículas $\eta_{0}$. Dizemos que uma partícula é livre desde o início se a partícula nunca encontra outra partícula com velocidade zero no seu caminho.

- Dizemos que uma partícula está permanentemente bloqueada no instante de tempo $t$ se sua velocidade é 0 para todos os instantes maiores o iguais a $t$.

- Dizemos que estamos em estado de fase de fluxo livre quando todas as partículas entram eventualmente no estado de fluxo livre

- Dizemos que estamos em fase de engarrafamento quando existe uma partícula que está bloqueada infinitas vezes. 
- O conjunto de sítios vazios(pode ser zero) entre duas partículas consecutivas é chamado de gap e o número de tais sítios é chamado de tamanho do gap. Se o tamanho de um gap é zero, diremos que a partícula mais a esquerda está bloqueada.

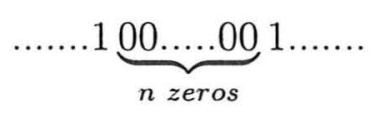

- Se duas partículas consecutivas estão separadas por um gap de tamanho zero, diremos que existe um bond.

- Mais de duas partículas unidas por gaps de tamanho zero serão chamados de cluster de partículas.

$$
\ldots .0 \underbrace{11 \ldots \ldots 11}_{n \text { uns }} 0 \ldots .
$$

- Buracos unidos um com outro entre os quais não existe nenhuma partícula serão chamados de cluster de buracos.

$$
\ldots 1 \underbrace{00 \ldots .00}_{n \text { zeros }} 1 \ldots
$$

Observação. Todas estas definições podem-se fazer também para os espaços livres(buracos), que serão chamados de anti-particulas, e assim teremos ant-igap, ant-ibond e clusters de anti-partículas definidas de forma equivalente.

\subsubsection{Propriedades do operador do autômato celular 184 atu- ando em configurações finitas}

Para entender a dinâmica do Autômato Celular 184 consideremos configurações finitas. Consideremos a configuração inicial $\eta_{o}$. Seja $n \in \mathbb{N}, n>0$. Uma configuração finita de tamanho $n$, denominada de bloco é o conjunto ordenado $\eta(1), \eta(2), \ldots \ldots \eta(n)$, onde $\eta(k) \in$ $\{0,1\}$. Este conjunto será denotado por $\mathcal{B}_{n}$ e definido por

$$
\mathcal{B}_{n}:=\left\{\eta_{0} \in \Omega:\left(\eta_{0}(1), \eta_{0}(2) \ldots ., \eta_{0}(n)\right)=\left(a_{1}, a_{2} \ldots ., a_{n}\right) ; a_{1} \ldots a_{n} \in\{0,1\}\right\}
$$

A função $f_{184}$ ou simplesmente $f$ é $f=\{0,1\}^{3} \longmapsto\{0,1\}$. Alternativamente pode ser considerada como a função de $\mathcal{B}_{3}$ em $\mathcal{B}_{1}=\{0,1\}$

Um operador de evolução atuando sobre configurações finitas correspondente à função local $f$ é uma função $\mathrm{h}: \mathcal{B}_{n} \longmapsto \mathcal{B}_{n-2}$ definida como 


$$
\begin{array}{r}
\mathbf{h}\left(\eta_{0}(1), \eta_{0}(2), \ldots, \eta_{0}(n)\right)=f\left(\eta_{0}(1), \eta_{0}(2), \eta_{0}(3)\right) \times \\
\times f\left(\eta_{0}(2), \eta_{0}(3), \eta_{0}(4)\right) \times \ldots \times f\left(\eta_{0}(n-2), \eta_{0}(n-1), \eta_{0}(n)\right)
\end{array}
$$

para $n>2$.

Definiremos a pré-imagem de um bloco de $a \in \mathcal{B}_{k}$ como um bloco $b \in B_{k+2}$ tal que $\mathbf{h}(b)=a$. Similarmente, uma pré-imagem de uma $n$-ésima interação de bloco $a \in \mathcal{B}_{k}$ é um bloco $b \in \mathcal{B}_{k+2 n}$ tal que $\mathbf{h}^{n}(b)=a$. Onde $\mathbf{h}^{n}=\underbrace{\mathbf{h} \circ \mathbf{h} \circ \ldots \circ \mathbf{h}}_{n \text { vezes }}$

Uma magnitude que caracteriza os modelos de trânsito é o fluxo das partículas.

Definição 2. Dada uma configuração $\eta_{t}$ do autômato celular 184, o fluxo, denotado por $\theta(\rho, t)$, é definido por

$$
\theta(\rho, t):=\mathbb{E}\left(V\left(\eta_{t}, i\right)\right) \forall i \in \mathbb{Z}
$$

onde $V\left(\eta_{t}, i\right)$ é a velocidade da partícula que está no ponto $i$ na configuração $\eta$ no instante $t$. $\mathbb{E}$ é a esperança definida com respeito a medida $\nu_{\rho}$.

O fluxo é o número de partículas cruzando um sítio fixo por unidade de tempo. Como as partículas não podem ultrapassar uma à outra e da invariância por translações temos que cada partícula tem velocidade média assintótica $v$. Desde que a densidade $\rho$ é a probabilidade que exista uma partícula na origem teremos que $v=\frac{\theta}{\rho}$, ou para o fluxo assintótico

$$
\theta(\rho)=\rho v
$$

Dada uma configuração $\eta_{t}$ no tempo $t$. Consideremos o bloco de tamanho $2 n+1$ denotado por $\mathcal{B}_{-n, n}$

$$
\mathcal{B}_{-n, n}=\left\{\eta_{t} \in \Omega:\left(\eta_{t}(-n), \eta_{t}(-n+1), \ldots, \eta_{t}(n)\right)=\left(a_{-n}, a_{-n+1} \ldots, a_{n}\right) ; a_{-n} \ldots . a_{n} \in\{0,1\}\right\}
$$

A configuração $\eta_{t} \in \mathcal{B}_{-n, n}$ será denotada por $\eta_{t}^{[-n, n]}$

Definição 3. A velocidade da partícula que está no ponto $i \in \mathbb{Z}$ na configuração $\eta_{t} \in \mathcal{B}_{-n, n}$ é definida por

$$
V\left(\eta_{t}^{[-n, n]}, i\right):=\eta_{t}(i)\left(1-\eta_{t}(i+1)\right):= \begin{cases}1 & \text { se } \eta_{t}(i) \eta_{t}(i+1)=10 \\ 0 & \text { caso contrario }\end{cases}
$$

Definição 4. A velocidade média no espaço das partículas na configuração $\eta_{t} \in \mathcal{B}_{-n, n}$ é definida por

$$
V\left(\eta_{t}\right):=\lim _{n \rightarrow \infty} V\left(\eta_{t}^{[-n, n]}\right)
$$


onde,

$$
V\left(\eta_{t}^{[-n, n]}\right):=\frac{1}{\sum_{i=-n}^{n} \eta_{t}(i)}\left(\sum_{i=-n}^{n-1} V\left(\eta_{t}^{[-n, n]}, i\right)\right)
$$

Definição 5. A velocidade média no tempo da partícula $C_{i}$, que está na posição $x_{i}$ no instante inicial $t=0$ é

$$
\bar{V}\left(\eta_{0}, x_{i}, t\right):=\frac{1}{t} L\left(\eta_{0}, x_{i}, t\right)
$$

$L\left(\eta_{0}, x_{i}, t\right)$ é a distância que a partícula $C_{i}$ avança durante $t$ instantes de tempo, estando inicialmente (em $t=0$ ) no ponto $x_{i}$ da configuração $\eta_{0}$.

A velocidade assintótica será:

$$
v:=V\left(C_{i}\right):=\lim _{t \rightarrow \infty} \bar{V}\left(\eta_{0}, x_{i}, t\right)
$$

\subsection{Resultados}

Teorema 1. Seja $\left\{\eta_{t}, t \in \mathbb{N}\right\}$ o autômato celular $184 \mathrm{com}$ distribuição inicial a medida produto de Bernoulli de densidade $\rho \in[0,1], \prod_{i \in \mathbb{Z}}\left\{\nu_{\rho}\left(\eta_{0}(i)=s\right)\right\}$ com $s \in\{0,1\}$. Com marginais iguais a $\nu_{\rho}\left(\eta_{0}(i)=1\right)=1-\nu_{\rho}\left(\eta_{0}(i)=0\right)=\rho$.

a. Se $0<\rho<\frac{1}{2}$, então, as partículas entram em fluxo livre com probabilidade 1 .

b. Se $\frac{1}{2} \leq \rho \leq 1$, então, uma partícula estará bloqueada infinitas vezes com probabilidade 1.

Teorema 2. Seja $\left\{\eta_{t}, t \in \mathbb{N}\right\}$ o autômato celular $184 \mathrm{com}$ distribuição inicial a medida produto de Bernoulli de densidade $\rho \in[0,1], \prod_{i \in \mathbb{Z}}\left\{\nu_{\rho}\left(\eta_{0}(i)=s\right)\right\}$ com $s \in\{0,1\}$. Com marginais iguais a $\nu_{\rho}\left(\eta_{0}(i)=1\right)=1-\nu_{\rho}\left(\eta_{0}(i)=0\right)=\rho$.

a. Se $0<\rho<\frac{1}{2}$, então, a velocidade assintótica das partículas será 1

b. Se $\frac{1}{2}<\rho<1$, então, a velocidade assintótica das partículas será $\frac{1}{\rho}-1$.

c. O fluxo de $\theta$ é dado por

$$
\theta(\rho):= \begin{cases}\rho & \text { se } 0<\rho<\frac{1}{2} \\ 1-\rho & \text { se } \frac{1}{2} \leq \rho<1\end{cases}
$$

Teorema 3. Seja $\left\{\eta_{t}, t \in \mathbb{N}\right\}$ o autômato celular $184 \mathrm{com}$ distribuição inicial a medida produto de Bernoulli de densidade $\rho=\frac{1}{2}, \prod_{i \in \mathbb{Z}}\left\{\nu_{1 / 2}\left(\eta_{0}(i)=s\right)\right\} \operatorname{com} s \in\{0,1\}$. Com marginais iguais a $\nu_{1 / 2}\left(\eta_{0}(i)=1\right)=1-\nu_{1 / 2}\left(\eta_{0}(i)=0\right)=\frac{1}{2}$. Então, a velocidade assintótica das partículas será 1 . 
Teorema 4. Seja $\left\{\eta_{t}, t \in \mathbb{N}\right\}$ o autômato celular 184 com distribuição inicial a medida produto de Bernoulli de densidade $\rho=\frac{1}{2}, \prod_{i \in \mathbb{Z}}\left\{\nu_{1 / 2}\left(\eta_{0}(i)=s\right)\right\}$ com $s \in\{0,1\}$. Com marginais iguais a $\nu_{1 / 2}\left(\eta_{0}(i)=1\right)=1-\nu_{1 / 2}\left(\eta_{0}(i)=0\right)=\frac{1}{2}$. Seja $\mu_{t}$ a distribuição de $\eta_{t}$. Então, $\mu_{t}$ converge para $\frac{1}{2}\left(\delta_{p}+\delta_{i}\right)$ quando $t \rightarrow \infty$ com decaimento da forma $t^{-\frac{1}{2}}$.

Onde $\delta_{p}$ é a medida concentrada na configuração que tem partículas com velocidade 1 nos números pares e buracos nos números ímpares de $\mathbb{Z}$ e $\delta_{i}$ é a medida concentrada na configuração que tem partículas nos números ímpares e buracos nos números pares de $\mathbb{Z}$.

\subsection{Autômato Celular 226}

O Autômato Celular 184 descreve a dinâmica das partículas. Quando a densidade é maior que $1 / 2$ é mais simples estudar a dinâmica dos buracos. Par fazer uso desta propriedade, introduzimos para uma configuração $\eta$ do autômato celular 184 a configuração dual $\eta^{*}(i):=1-\eta(i) \forall i \in \mathbb{Z}$. Definimos o Autômato Celular 226 no mesmo espaço que do AC 184 pela dinâmica do operador dual $F^{*}:\{0,1\}^{\mathbb{Z}} \rightarrow\{0,1\}^{\mathbb{Z}}$ tal que $\eta_{t+1}=F^{*} \eta_{t}$. Se $\eta_{t} \in\{0,1\}^{\mathbb{Z}}$ representa a configuração do autômato em um arbitrário instante de tempo $t, t \in \mathbb{N}=\{0,1,2 \ldots\}$, então, $\eta_{t+1} \in\{0,1\}^{\mathbb{Z}}$ é definido por

$$
\eta_{t+1}(i)=F^{*} \eta_{t}(i):= \begin{cases}0 & \text { se } \eta(i+1)=\eta(i)=0 \\ 0 & \text { se } \eta(i)=1-\eta(i-1)=1 \\ 1 & \text { caso contrário }\end{cases}
$$

para todo $i \in \mathbb{Z}$.

Lema 0.

$$
F \eta=\left(F^{*} \eta^{*}\right)^{*}
$$

Prova. Imediata da definição do operador dual.

\section{Corolário 0.}

$$
(F \eta)^{*}=\left(F^{*} \eta^{*}\right)
$$

Prova. Evidente.

\subsection{Processo de Aniquilação Balística}

Este processo denotado por PAB, também pode ser encontrado na literatura como Aniquilação Balística ou processo de reação e aniquilação $A+B \mapsto$ inerte. Introduzimos este modelo pela sua estreita relação com o AC 184, que será evidente depois de enunciar o próximo lema 1. O PAB tem três estados $-1,0,1$ e a seguinte regra de evolução: Ao tempo $t=0$ partículas são distribuídas em todos os sítios de $\mathbb{Z}$, ao mais uma partícula por sítio. Representamos um sítio ocupado por uma partícula por -1 e 1 e vazio por 0 . Escolhemos a velocidade das partículas com a medida produto de Bernoulli $\nu_{\rho}$ de densidade $\rho=\frac{1}{2}$, o qual 
distribui para cada partícula a velocidade -1 ou 1 , independentemente com probabilidade $\frac{1}{2}$.

Do tempo $t$ para o tempo $t+1$ as partículas com velocidade 1 se movem para a direita ao largo de $\mathbb{R}$ e serão ditas de partículas de classe-A; e as partículas de velocidade -1 que se movem em sentido contrário, serão ditas de partículas de classe-B. Se uma partícula de classe-A encontra no seu caminho uma partícula de classe-B, estas colidem e se aniquilam. Após a aniquilação as partículas desaparecem do processo para sempre.

A dinâmica do PAB será definida pelo operador $A:\{-1,0,1\}^{\mathbb{Z}} \rightarrow\{-1,0,1\}^{\mathbb{Z}}$ tal que $\xi_{t+1}=A \xi_{t}$. Se $\xi_{t} \in\{-1,0,1\}^{\mathbb{Z}}$ representa a configuração do autômato em um arbitrário instante de tempo $t, t \in \mathbb{N}=\{0,1,2 \ldots\}$, então, $\xi_{t+1} \in\{-1,0,1\}^{\mathbb{Z}}$ é definido por $\xi_{t+1}=A \xi_{t}:=$

$$
\xi_{t+1}(i):= \begin{cases}1 & \text { se } \xi(i-1)=1 \text { e nem } \xi(i)=-1 \text { nem } \operatorname{ambos} \xi(i)=0, \xi(i+1)=-1 \\ -1 & \text { se } \xi(i+1)=-1 \text { e nem } \xi(i)=1 \text { nem } \operatorname{ambos} \xi(i)=0, \xi(i-1)=1 \\ 0 & \text { caso contrário }\end{cases}
$$

para todo $i \in \mathbb{Z}$.

\section{Lema 1. Relação entre AC 184 e PAB}

Consideremos os modelos CA 184 e o PAB e definamos a função

$T_{184, A B}:\{0,1\}^{\mathbb{Z}} \rightarrow\{-1,0,1\}^{\mathbb{Z}}$ por

$$
\left(T_{184, B A} \eta\right)(i)=1-\eta(i)-\eta(i-1)
$$

para todo $i \in \mathbb{Z}$. Seja $\eta, \hat{\eta} \in\{0,1\}^{\mathbb{Z}}$ e $\xi, \hat{\xi} \in\{-1,0,1\}^{\mathbb{Z}}$ tal que $T_{184, B A}(\eta)=\xi$ e $T_{184, B A}(\hat{\eta})=\hat{\xi}$. Então, $\hat{\eta}=F \eta$ se somente se $\hat{\xi}=A \xi$.

Prova. Está no artigo de Belitsky e Ferrari[6], Lemma 1.

\subsection{Modelo de Crescimento de Superfícies}

Este modelo denotado por MCS é um processo a tempo discreto com valores em $\mathcal{R}$. Onde $\mathcal{R}$ é o espaço de funções contínuas de $\mathbb{R}$ em $\mathbb{R}$ com inclinação de $45^{\circ}$ ou $315^{\circ}$ entre qualquer duas abscissas inteiras. $\mathrm{O}$ valor que alcança em cada abscissa inteira é também inteiro (ver figura 2.1).

A dinâmica do MCS será definida pélo operador $S: \mathcal{R} \rightarrow \mathcal{R}$ tal que $f_{t+1}()=.S f_{t}$. Se $f_{t}(.) \in \mathcal{R}$ representa a configuração de $\mathcal{R}$, no instante de tempo $t, t \in \mathbb{N}=\{0,1,2 \ldots\}$, então, $f_{t+1}(.) \in \mathcal{R}$ é definido por

$$
f_{t+1}(.)=S f_{t}(.):=\left\{\begin{array}{cl}
f(i)+2, & \text { se } f(i)=f(i+1)-1=f(i-1)-1 \\
f(i), & \text { caso contrário }
\end{array}\right.
$$



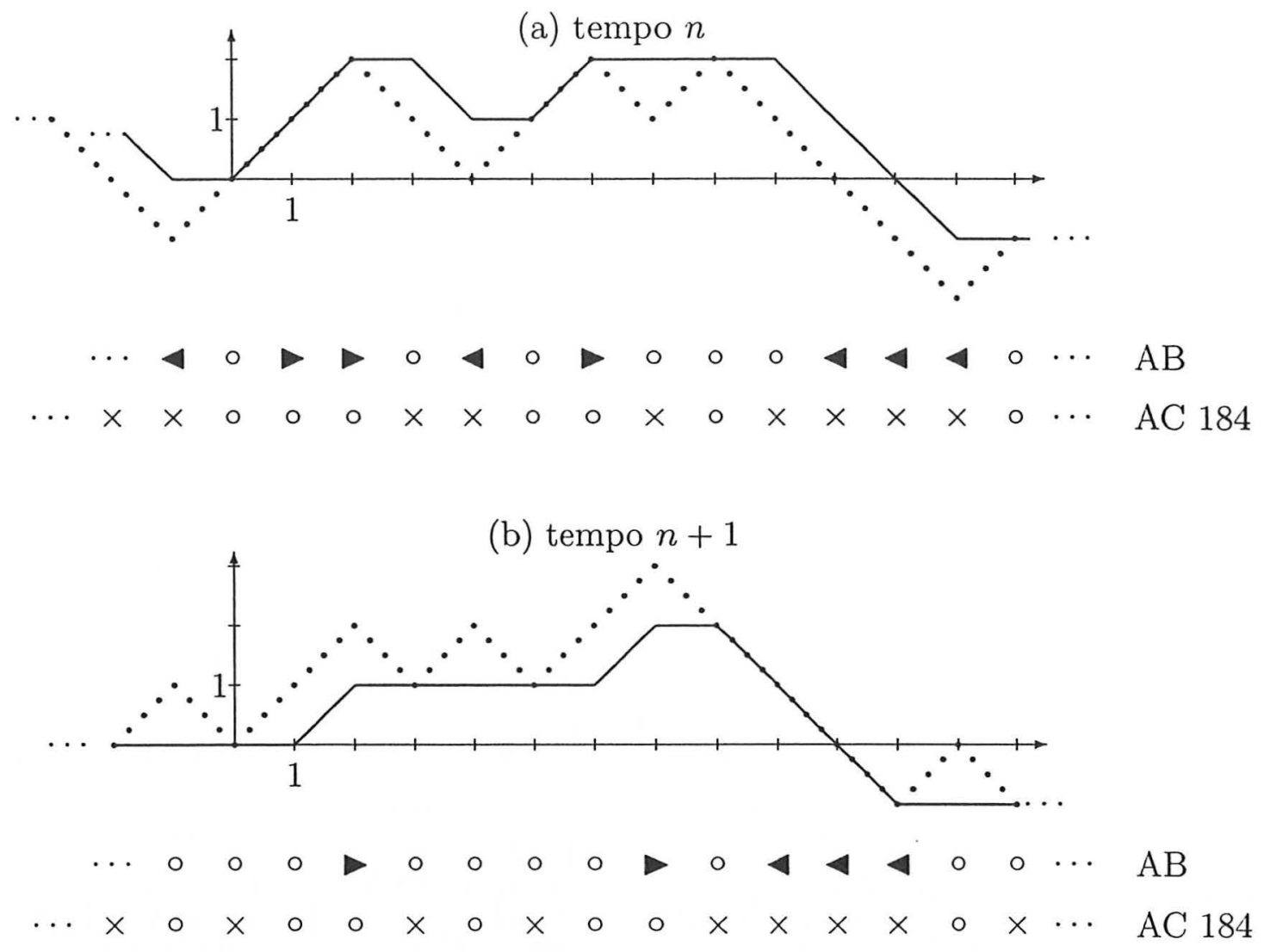

Figura 2.1: Evolução do autômato celular 184, do processo de aniquilação balística e do modelo de crescimento de superfícies. Construção dos respectivos perfis para AC 184 e o PAB e ilustração dos lemas 1 e 2

para todo $i \in \mathbb{Z}$.

Lema 2. Relação entre CA 184 e MCS.

Consideremos os modelos CA 184 e MCS e definamos

$T_{C S, 184}: \mathcal{R} \rightarrow\{0,1\}^{\mathbb{Z}}$ por

$$
\left(T_{C S, 184} f\right)(i)= \begin{cases}0 & \text { se } f(i)-f(i-1)=1 \\ 1 & \text { se } f(i)-f(i-1)=-1\end{cases}
$$

para todo $i \in \mathbb{Z}$.

Seja $f, \hat{f} \in \mathcal{R}$ e $\eta, \hat{\eta} \in\{0,1\}^{\mathbb{Z}}$ tal que $T_{C S, 184} f=\eta$ e $T_{C S, 184} \hat{f}=\hat{\eta}$. Então, $\hat{\eta}=F \eta$ se, somente se, a função $\hat{f}$ e $S f$ têm a mesma forma, isto é, se, somente se, $(S f)(x)-(S f)(0)=$ $\hat{f}(x)-\hat{f}(0)$, fora todo $x \in \mathbb{R}$. 
Prova. Está no artigo de Belitsky e Ferrari[6], Lemma 2.

Comentário da figura. Na figura, nas linhas inferiores às figuras (a) e (b), apresentamos um pedaço da configuração do $\mathrm{AC} 184$ sobre $\mathbb{Z}$, onde as partículas são representadas por uma $\times$ e os sítios vazios são representados por uma o. A configuração em (b) é obtida pela dinâmica do AC 184. Nas linhas superiores das figuras (a) e (b) apresentamos um pedaço da configuração do $\mathrm{PAB}$ sobre $\mathbb{Z}$, onde os sítios que contêm partículas positivas (respectivamente, negativas )A-partículas (B-partículas) são representados por 4 (respectivamente, $\triangleright$ ), os sítios vazios são representado por uma o. A configuração em (b) é obtida pela dinâmica do PAB.

Os pontos em (a) e (b) representam as figuras para o MCS sobre $\mathbb{R}$. A função em (b) é obtida pela dinâmica do MCS. Figuras sólidas em (a) e (b) representam perfis para $\mathrm{PAB}$ no sentido da fórmula 2.19. Figuras de pontos (a) e (b) representam os perfis para CA 184 no sentido da fórmula 2.21.

Em ambas figuras (a) e (b), a configuração de PAB (respectivamente MCS) é obtida usando a configuração de AC 184 mediante a fórmula 2.19 (respectivamente 2.21); a preservação desta relação no passo da figura (a) para a figura (b) ilustra o lema 1 (respectivamente 2).

\subsection{Prova dos Teoremas}

Enunciaremos de novo os teoremas.

Teorema 1. Seja $\left\{\eta_{t}, t \in \mathbb{N}\right\}$ o autômato celular $184 \mathrm{com}$ distribuição inicial a medida produto de Bernoulli de densidade $\rho \in[0,1], \prod_{i \in \mathbb{Z}}\left\{\nu_{\rho}\left(\eta_{0}(i)=s\right)\right\}$ com $s \in\{0,1\}$. Com marginais iguais a $\nu_{\rho}\left(\eta_{0}(i)=1\right)=1-\nu_{\rho}\left(\eta_{0}(i)=0\right)=\rho$.

a. Se $0<\rho<\frac{1}{2}$, então, as partículas entram em fluxo livre com probabilidade 1 .

b. Se $\frac{1}{2} \leq \rho \leq 1$, então, uma partícula estará bloqueada infinitas vezes com probabilidade 1.

Para provar o teorema precisamos dos seguintes lemas.

Lema 3. Seja $\left\{\eta_{t}, t \in \mathbb{N}\right\}$ o autômato celular 184 com distribuição inicial a medida produto de Bernoulli de densidade $\rho \in[0,1], \prod_{i \in \mathbb{Z}}\left\{\nu_{\rho}\left(\eta_{0}(i)=s\right)\right\}$ com $s \in\{0,1\}$. Com marginais iguais a $\nu_{\rho}\left(\eta_{0}(i)=1\right)=1-\nu_{\rho}\left(\eta_{0}(i)=0\right)=\rho$.

Dada uma configuração $\eta_{0}$ no estado inicial. Consideremos o intervalo $[x+1, x+n] \cap \mathbb{Z}$ de longitude $n\left(\eta_{0}\right)$ suficientemente grande e $x>0, x \in \mathbb{Z}$. Seja $X_{n}$ o número de bonds e $Y_{n}$ o número de anti-bonds neste intervalo. Se $0<\rho<\frac{1}{2}$, então existe um $N\left(\eta_{0}\right)$ tal que com probabilidade 1 teremos menos bonds que anti-bonds. Ademais,

$$
\frac{X_{n}-Y_{n}}{n} \rightarrow \frac{1}{2}(2 \rho-1) \quad \forall n \geq N\left(\eta_{0}\right)
$$


com probabilidade 1 .

Prova. É direta usando a lei dos grandes números.

Lema 4 [Relação entre AC 184 e o PAB].

Consideremos um anti-bond (..1001...) e um bond (...0110...). Então, existe uma partícula de classe-A (...0A...) associado ao anti-bond (...1001...) e uma partícula de classe$\mathrm{B}(\ldots 0 B \ldots)$ associado ao bond $(\ldots 0110 \ldots)$.

\section{Prova.}

Consideremos os modelos CA 184 e o PAB e do lema 1 temos que estes dois modelos estão relacionados pela função

$T_{184, B A}:\{0,1\}^{\mathbb{Z}} \rightarrow\{-1,0,1\}^{\mathbb{Z}}$ por

$$
\left(T_{184, B A} \eta\right)(i)=1-\eta(i)-\eta(i-1)
$$

Usando o operador $T_{184, A B}$ conseguimos

$$
T_{184, A B}(\ldots 1001 \ldots)=(\ldots 0 A \ldots) \text { e } T_{184, A B}(\ldots 0110 \ldots)=(\ldots 0 B \ldots)
$$

Observação Os anti-bonds (partículas de classe-A) viajam à direita à velocidade 1 e os bonds (partículas de classe-B) viajam à esquerda com velocidade 1 .

Lema 5. Supondo que um cluster de anti-bonds de tamanho $r$ se encontra com um cluster de bonds de tamanho $s$. Se $r>s$, então, o cluster de anti-bonds aniquila o cluster de bonds.

Prova. A prova é direta do lema 4 e da definição do partículas de classe-A e Classe-B.

Temos que $T_{184, A B}\left(\ldots 10_{r} 1 \ldots\right)=\left(\ldots 0 A_{r-1} 0 \ldots\right)$ e $T_{184, A B}\left(\ldots 01_{s} 0 \ldots\right)=\left(\ldots 0 B_{s-1} 0 \ldots\right)$

Logo $T_{184, A B}\left(\ldots 10_{r} 1_{s} 0\right)=\left(\ldots 0 A_{r-1} 0 B_{s-1} \ldots\right)$. Então, como $r>s$ todos os bonds são aniquilados.

Lema 6. Seja $\left\{\eta_{t}, t \in \mathbb{N}\right\}$ o autômato celular $184 \mathrm{com}$ distribuição inicial a medida produto de Bernoulli de densidade $\rho \in[0,1], \prod_{i \in \mathbb{Z}}\left\{\nu_{\rho}\left(\eta_{0}(i)=s\right)\right\}$ com $s \in\{0,1\}$. Com marginais iguais a $\nu_{\rho}\left(\eta_{0}(i)=1\right)=1-\nu_{\rho}\left(\eta_{0}(i)=0\right)=\rho$.

Dada uma configuração $\eta_{0}$ no estado inicial. Consideremos o intervalo $[x+1, x+n] \cap \mathbb{Z}$ de longitude $n\left(\eta_{0}\right)$ suficientemente grande e $x>0, x \in \mathbb{Z}$. Assumindo que todos cluster de anti-bonds vão ao encontro (estão à esquerda) de todos os cluster de bonds. Se $0<\rho<1 / 2$, então, todos os bonds são destruídos com probabilidade 1 em um tempo finito que não é maior que o número de partículas dentro do intervalo.

Prova. Considerando que os anti-bonds estão sempre na esquerda dos bonds. Do lema 3 temos que o número de anti-bonds(dois buracos juntos) é maior que o número de bonds(duas partículas juntas). Usando a dinâmica do AC 184 e o lema 4, temos que o 
número de partículas de classe-A é maior que as partículas de classe-B. Portanto, usando o Lema 5 teremos que os bonds (partículas de classe-B) são aniquilados com probabilidade 1 .

Observação. Caso os cluster de bonds estejam sempre à esquerda dos cluster de anti-bonds, não é necessário usar o operador $T_{184, A B}$ porque podemos usar a seguinte propriedade:

$$
\underbrace{111 \ldots \ldots 111}_{\text {muns }} \underbrace{000 \ldots \ldots .000}_{n \text { zeros }}, m<n
$$

o cluster de uns desaparece depois de $(m-1)$ iterações.

Portanto, todos os bonds também serão aniquilados.

Estes lemas nos permitem dar condições suficientes e necessárias para que uma partícula entre em estado de fluxo livre.

Proposição 1. Seja $\left\{\eta_{t}, t \in \mathbb{N}\right\}$ o autômato celular 184 com distribuição inicial $\eta_{0}$.

A condição necessária e suficiente para que uma partícula, na origem, entre em fluxo livre é

$$
\exists k\left(\eta_{0}\right): 2 \sum_{i=1}^{k} \eta(i)<k, \quad \forall k \geq k\left(\eta_{0}\right)
$$

Prova da proposição 1. Considere o bloco finito de tamanho $2 n+2$ que começa no ponto $m>0$.

$$
\mathcal{B}_{2 n+2}=\left\{\eta \in \Omega:(\eta(m+1), \eta(m+2) \ldots ., \eta(m+2 n+2))=\left(a_{1}, a_{2} \ldots ., a_{2 n+2}\right)\right\}
$$

onde $\left\{a_{1} \ldots a_{2 n+2} \in\{0,1\}\right\}$ e $n>m>0$.

Provemos primeiro que se existe um $m=m\left(\eta_{0}\right)$ tal que

$$
2 \sum_{i=m+1}^{m+k} \eta(i)<k, \text { para } 1 \leq k \leq 2 n+2, n>m
$$

Então,

$$
\mathbf{h}^{n}\left(\eta_{0}(m+1), \eta_{0}(m+2) \ldots ., \eta_{0}(m+2 n+2)\right)=00 .
$$

Para isto usemos a relação do AC 184 com o Modelo de Crescimento de Superfícies, definido no lema 2. Consideremos os modelos CA 184 e MCS e definamos

$T_{C S, 184}: \mathcal{R} \rightarrow\{0,1\}^{\mathbb{Z}}$ por

$$
\left(T_{C S, 184} f\right)(i)= \begin{cases}0 & \text { se } f(i)-f(i-1)=1 \\ 1 & \text { se } f(i)-f(i-1)=-1\end{cases}
$$

Para toclo $i \in \mathbb{Z}$. 
Definamos $\zeta(i):=f(i)-f(i-1)$ para todo $i \in \mathbb{Z}$. Com isto, usando a relação acima teremos

$$
\zeta(i)=1-2 \eta(i)= \begin{cases}1 & \text { se } \eta(i)=0 \\ -1 & \text { se } \eta(i)=1\end{cases}
$$

A equação 2.26 se transforma em

$$
\sum_{i=m+1}^{m+k} \zeta(i)>0 \text { para } 1 \leq k \leq 2 n+2, n>m
$$

Da representação gráfica do modelo de crescimento de superfícies, vemos que isto acontece se, somente se, no intervalo $[m+1, m+2 n+2] \cap \mathbb{Z}$ para cada $k$ tal que $1 \leq k \leq$ $2 n+2$ o número de anti-bonds é maior que o número de bonds. Ademais, necessariamente $\eta_{0}(m+1)=\eta_{0}(m+2)=0$. Passando a configuração inicial à configuração do modelo de aniquilação balística, teremos que pelo lema 6 todos os bonds que existem serão aniquilados com probabilidade 1 . Como resultado da evolução teremos que depois de $n$ interações encontraremos só o bloco 00, isto é, $\eta_{n}(m+n+1) \eta_{n}(m+n+2)=00$. Logo, temos

$$
\mathbf{h}^{n}\left(\eta_{0}(m+1), \eta_{0}(m+2) \ldots . ., \eta_{0}(m+2 n+2)\right)=00
$$

Então, existe um $k\left(\eta_{0}\right)$ tal que a partir do momento $t_{0}\left(\eta_{0}\right):=k\left(\eta_{0}\right) \leq m$, a partícula na origem no instante inicial ficará livre para sempre.

Provemos a volta. Se a partícula na origem está livre para sempre, então, existe um $t_{0}\left(\eta_{0}\right)$ tal que a partir deste tempo a partícula $C_{0}$ estará livre. Teremos que a partir do ponto $x_{0}(t)$ (que é a posição desta partícula no tempo $t_{0}$ ) a seguinte relação cumpre-se

$$
\exists k\left(\eta_{0}\right)=t_{0}\left(\eta_{0}\right): 2 \sum_{i=x_{0}(t)+1}^{x_{0}(t)+k} \eta(i)<k, \quad \forall k \geq k\left(\eta_{0}\right)
$$

A partir do ponto $x_{0}\left(t_{0}\right)$ à direita todas as partículas são livres. Portanto, existe ao menos uma partícula $C_{j}$ na posição $x_{j}\left(t_{0}\right)=x_{j}+t_{0}$ que é livre desde o início. Esta partícula na configuração inicial tinha a posição $x_{j}$. Portanto, se a partícula na origem é livre, então, existe um $k\left(\eta_{0}\right)=x_{j}$ tal que,

$$
\mathrm{h}^{t_{0}}\left(\eta_{0}\left(x_{j}+1\right), \eta_{0}\left(x_{j}+2\right) \ldots ., \eta_{0}\left(x_{j}+2 t_{0}+2\right)\right)=00
$$

Vemos que isto acontece se, somente se, $\eta_{n}\left(x_{j}+n+1\right) \eta_{n}\left(x_{j}+n+2\right)=00$ usando a transformação no modelo de aniquilação balística para este sub-bloco teríamos $0 A$. Desde que as partículas de classe-A movem-se à direita à velocidade 1 , isto significa que no instante $t=0$ deveríamos de ter $0 \mathrm{~A}$ ou usando $\mathrm{AC} 184 \eta_{0}\left(x_{j}+1\right) \eta_{0}\left(x_{j}+2\right)=00$. O que significa que a partícula de classe-A viaja de $i=x_{j}+3$ até $i=x_{j}+t_{0}+2$ em $t_{0}$ unidades de tempo. Esta partícula pode viajar esta distância sem ser aniquilada se, somente se, não colide com uma partícula de classe- $\mathrm{B}$ que se move em sentido contrário. Isto pode acontecer se todas as partículas de classe-B são aniquiladas antes de encontrarem-se com esta partícula de classe- $\mathrm{A}$, isto é , se para cada $x_{j}+3 \leq k \leq x_{j}+2 t_{0}+2$ o número de 
partículas de classe-B (bonds) dentro do sub-bloco $\eta_{0}\left(x_{j}+3\right), \eta_{0}\left(x_{j}+4\right) \ldots ., \eta_{0}\left(x_{j}+2 t_{0}+2\right)$ é menor que o número de partículas de classe-A (anti-bonds). Usando a notação de zeros e uns temos

$$
\begin{gathered}
\mathbf{h}^{t_{0}}\left(\eta_{0}\left(x_{j}+1\right), \eta_{0}\left(x_{j}+2\right) \ldots \ldots, \eta_{0}\left(x_{j}+2 t_{0}+2\right)\right)=00 \Longrightarrow \\
\sum_{i=x_{j}+1}^{x_{j}+k} \zeta(i)>0 \text { para } 1 \leq k \leq 2 t_{0}+2
\end{gathered}
$$

Portanto, se a partícula na origem está em fluxo livre temos que

$$
\sum_{i=x_{j}+1}^{x_{j}+k} \zeta(i)>0 \quad \forall k, 1 \leq k \leq 2 t_{0}+2
$$

Observação. Dada a condição

$$
2 \sum_{i=m+1}^{m+k} \eta_{0}(i)<k, \quad \forall k>1
$$

Se existe uma partícula no ponto $m$, então, esta partícula será livre desde o início.

\section{Prova do Teorema 1.}

Parte a. Como $0<\rho<\frac{1}{2}$, pela lei dos grande números com probabilidade 1 , cumprese

$$
\exists N\left(\eta_{0}\right):\left|\frac{1}{n} \sum_{i=1}^{n} \eta(i)-\rho\right|<\epsilon, \forall n>N\left(\eta_{0}\right), \forall \epsilon>0
$$

Trabalhando um lado da desigualdade, temos

$$
\frac{1}{n} \sum_{i=1}^{n} \eta(i)<(\rho+\epsilon)
$$

Como $\epsilon>0$ é arbitrário, escolhemos $\epsilon$ tal que $\epsilon<1 / 2-\rho$. Com isto conseguimos

$$
\exists N\left(\eta_{0}\right): 2 \sum_{i=1}^{k} \eta(i)<k, \quad \forall k \geq N\left(\eta_{0}\right)
$$

Usando a proposição 1 o resultado é direto. Portanto, teremos fluxo livre.

Parte b. Para $1 / 2 \leq \rho<1$ as partícula não entram em fluxo livre, esta é uma conseqüência direta da lei dos grandes números e da equação 2.32. Temos que para 
$1 / 2 \leq \rho<1$ a condição para qualquer partícula entrar em fluxo livre não se cumpre. Portanto, as partículas, neste caso, serão bloqueadas infinitas vezes. Agora, no autômato 226 (dual do AC 184) o comportamento das partículas é equivalente ao comportamento dos buracos. Para provar que uma partícula está bloqueada infinitas vezes basta provar que os buracos entram em fase de fluxo livre com probabilidade 1.

Provar que os buracos entram em fluxo livre é simples se estudarmos os buracos no Autômato Celular 226 e repetirmos o feito na parte a. Não faremos a prova porque seria repetir a prova da parte a.

Teorema 2. Seja $\left\{\eta_{t}, t \in \mathbb{N}\right\}$ o autômato celular $184 \mathrm{com}$ distribuição inicial a medida produto de Bernoulli de densidade $\rho \in[0,1], \prod_{i \in \mathbb{Z}}\left\{\nu_{\rho}\left(\eta_{0}(i)=s\right)\right\}$ com $s \in\{0,1\}$. Com marginais iguais a $\nu_{\rho}\left(\eta_{0}(i)=1\right)=1-\nu_{\rho}\left(\eta_{0}(i)=0\right)=\rho$.

a. Se $0<\rho<\frac{1}{2}$, então, a velocidade assintótica das partículas será 1

b. Se $\frac{1}{2}<\rho<1$, então, a velocidade assintótica das partículas será $\frac{1}{\rho}-1$.

c. O fluxo de $\theta$ é dado por

$$
\theta(\rho):= \begin{cases}\rho & \text { se } 0<\rho<\frac{1}{2} \\ 1-\rho & \text { se } \frac{1}{2} \leq \rho<1\end{cases}
$$

\section{Prova.}

Parte a. Pela parte a do teorema 1 temos que se $0<\rho<\frac{1}{2}$ qualquer partícula entra em fluxo livre. Consideremos a partícula $C_{n}$. Existe um tempo $t_{n}:=t_{n}\left(\eta_{0}\right)$ a partir do qual a velocidade desta partícula será 1.

Calculando a velocidade assintótica teremos:

$$
v=\lim _{t \rightarrow \infty} \frac{1}{t} L\left(\eta_{0}, x_{n}, t\right)
$$

Para qualquer partícula que inicialmente se encontra no ponto $x_{n} \in \mathbb{Z}$, a distância total $L\left(\eta_{0}, x_{n}, t\right)$ que avança durante o tempo $t$ é o número de buracos que $C_{n}$ encontrará no seu caminho durante este tempo. Esta quantidade pode ser expressa por

$$
L\left(\eta_{0}, x_{n}, t\right)=\sum_{s=0}^{t-1} 1\left\{V\left(C_{n}, s\right)=1\right\}
$$

onde $V\left(C_{n}, t\right)$ é a velocidade da partícula $C_{n}$ no instante $t$. Com isto, teremos

$$
v=\lim _{t \rightarrow \infty}\left\{\frac{1}{t} \sum_{s=0}^{t_{n}} L\left(\eta_{0}, x_{n}, s\right)+\frac{1}{t} \sum_{s=t_{n}+1}^{t-1} L\left(\eta_{0}, x_{n}, s\right)\right\}=1
$$

A primeira soma é zero. Na segunda soma temos que para $s>t_{n}$ a partícula $C_{n}$ está livre, portanto, em cada passo encontra um buraco. Logo, a velocidade assintótica é $v=1$. 
Parte b. Se $\frac{1}{2}<\rho<1$ calculemos a velocidade assintótica da partícula $C_{n}$.

$$
v=\lim _{t \rightarrow \infty} \frac{1}{t} L\left(\eta_{0}, x_{n}, t\right)
$$

$L\left(\eta_{0}, x_{n}, t\right)$ é o número de buracos que $C_{n}$ encontra no seu caminho e é dada por

$$
L\left(\eta_{0}, x_{n}, t\right)=\sum_{s=0}^{t-1} 1\left\{V\left(C_{n}, s\right)=1\right\}
$$

Denotemos por $b_{n, t}$ a posição original (no instante $t=0$ ) na configuração $\eta_{0}$ do último buraco que a partícula $C_{n}$ encontra em seu caminho durante o tempo $t$. Então, no bloco $\mathcal{B}_{\left[x_{n}, b_{n, t}\right]}$ há exatamente $L\left(\eta_{0}, x_{n}, t\right)$ buracos e podemos estabelecer a equação

$$
L\left(\eta_{0}, x_{n}, t\right)=\left(b_{n, t}-x_{n}\right) \cdot\left(1-\rho_{t}\right)
$$

Além disso o número de partículas neste bloco, denotado por $N_{t}$, será

$$
N_{t}=\left(b_{n, t}-x_{n}\right) \cdot \rho_{t}
$$

Aqui, $\rho_{t}$ é a densidade das partículas no bloco considerado.

A velocidade média será

$$
\bar{V}\left(\eta_{0}, x_{n}, t\right)=\frac{1}{t} L\left(\eta_{0}, x_{n}, t\right)=\frac{N_{t}}{t} \times \frac{1-\rho_{t}}{\rho_{t}}
$$

Tomando limite temos

$$
v=\frac{1-\rho}{\rho} \times \lim _{t \rightarrow \infty} \frac{N_{t}}{t}
$$

Clamamos que $\lim _{t \rightarrow \infty} \frac{N_{t}}{t}=1$. Para calcular este limite usaremos o autômato celular 226. Para este autômato cada buraco entra eventualmente em fase de trânsito livre. Portanto, para um buraco colocado no ponto $b_{n, t}$ que pertence ao mesmo bloco, temos que $N_{t}$ é a quantidade de partículas que encontrará no seu caminho, mas a partir de certo tempo este buraco estará livre e portanto, o limite deve de ser 1. Logo, a velocidade assintótica é $v=\frac{1}{\rho}-1$.

Parte c. O fluxo assintótico é definido por $\theta(\rho)=\rho v$, onde $v=\lim _{t \rightarrow \infty} \frac{1}{t} \sum_{s=0}^{t-1} 1\left\{V\left(\eta_{o}, t\right)\right\}$ é a velocidade assintótica. Da parte $\mathbf{a}$ e $\mathbf{b}$ foram calculadas as velocidades assintóticas

$$
v= \begin{cases}1 & \text { se } 0<\rho<\frac{1}{2} \\ \frac{1-\rho}{\rho} & \text { se } \frac{1}{2} \leq \rho<1\end{cases}
$$

Com isto a prova está concluída.

Teorema 3. Seja $\left\{\eta_{t}, t \in \mathbb{N}\right\}$ o autômato celular 184 com distribuição inicial a medida produto de Bernoulli de densidade $\rho=\frac{1}{2}, \prod_{i \in \mathbb{Z}}\left\{\nu_{1 / 2}\left(\eta_{0}(i)=s\right)\right\}$ com $s \in\{0,1\}$. Com marginais iguais a $\nu_{1 / 2}\left(\eta_{0}(i)=1\right)=1-\nu_{1 / 2}\left(\eta_{0}(i)=0\right)=\frac{1}{2}$. Então a velocidade 
assintótica das partículas será 1.

Para provar o teorema necessitamos do seguinte:

Lema 7. Seja $\left\{\xi_{n}\right\}_{n \in \mathbb{N}}$ uma seqüência de variáveis aleatórias no espaço de probabilidade definido para o $\mathrm{AC}$ 184. Seja $\left\{\epsilon_{n}\right\}_{n \geq 1}$ uma seqüência de números positivos tal que $\epsilon_{n} \downarrow 0$, quando $n \rightarrow \infty$. Se

$$
\sum_{n=1}^{\infty} \nu_{\rho}\left\{\left|\xi_{n}-\xi\right| \geq \epsilon_{n}\right\}<\infty
$$

Então, $\xi_{n} \rightarrow \xi$ quase certamente.

Prova. Está provado no Shiryaev[28] pag 256, corolário 2. A prova está referida a variáveis aleatórias genéricas.

Observação. Daremos sem demonstração que da definição 4

$$
\lim _{t \rightarrow \infty} V\left(\eta_{t}\right)=v \quad \nu_{\rho} \text { quase certamente }
$$

Prova do teorema 3. Consideremos um bloco de tamanho $2 n+1$ ( $n$ suficientemente grande) $\mathcal{B}_{[-n, n]}$ do espaço das configurações no tempo arbitrário $t$. Seja $\eta_{t}^{[-n, n]}$ uma configuração deste bloco. O objetivo da prova é encontrar duas configurações a partir de $\eta_{t}^{[-n, n]}$. Uma denotada por $\eta_{-}^{[-n, n]}$, que será obtida extraindo partículas da configuração $\eta_{t}^{[-n, n]}$. Com isto, a densidade dentro do bloco será menor que $1 / 2$ e quando $t \rightarrow \infty$ e $n \rightarrow \infty$ as partículas entram em fluxo livre e, portanto, a velocidade assintótica será 1. A outra configuração é denotada por $\eta_{+}^{[-n, n]}$, que será obtida agregando partículas à configuração $\eta_{t}^{[-n, n]}$. Com isto, a densidade dentro do bloco será maior que $1 / 2$ e pode-se provar que quando $t \rightarrow \infty$ e $n \rightarrow \infty$ a velocidade assintótica desta configuração será 1. Com esta operação de tirar e colocar partículas temos, na verdade, estabelecido uma relação de ordem entre as configurações. Tal que se cumpre

$$
\eta_{-}^{[-n, n]}(i) \leq \eta_{t}^{[-n, n]}(i) \leq \eta_{+}^{[-n, n]}(i), \quad \forall i \in \mathbb{Z}, \quad t \in \mathbb{R}_{+}
$$

As velocidades destas configurações mantêm a ordem contrária, porque a maior quantidade de partículas diminui à velocidade média da configuração. O número maior de partículas numa configuração só atrapalha o movimento de todas as outras partículas.

No limite quando $n \rightarrow \infty$ temos

$$
\eta_{-}(i) \leq \eta_{t}(i) \leq \eta_{+}(i), \quad \forall i \in \mathbb{Z}, \quad t \in \mathbb{R}_{+}
$$

Calculando a velocidade média assintótica teremos.

$$
1=\lim _{t \rightarrow \infty} \bar{V}\left(\eta_{+}\right) \leq \lim _{t \rightarrow \infty} \bar{V}\left(\eta_{t}\right) \leq \lim _{t \rightarrow \infty} \bar{V}\left(\eta_{-}\right)=1
$$


Para calcular a velocidade assintótica do lado esquerdo usaremos o lema 7 . Na configuração $\eta_{t}$, para $n$ grande, pela lei forte dos grandes números teremos.

$$
\exists N\left(\eta_{0}\right):\left|\frac{\sum_{i=-n}^{n} \eta_{t}(i)}{2 n+1}-\frac{1}{2}\right|<\epsilon, \forall n \geq N, \forall \epsilon>0
$$

Dado $n$ podemos escolher o número positivo $m$ e $k$ inteiro tal que $2 n+1=k m+l$, com $l \in\{-m,-m+1, \ldots . m-1, m\}$. Encontramos a distribuição $\eta_{+}^{[-n, n]}$ agregamos $k$ partículas nos $k$ buracos que estão enumerados antes da coordenada $\mathrm{km}$. Com isto, temos

$$
\left|\frac{\sum_{i=-n}^{n} \eta_{+}(i)}{2 n+1}-\left(\frac{1}{2}+\frac{1}{m}\right)\right| \leq\left|\frac{\sum_{i=-n}^{n} \eta_{t}(i)}{2 n+1}-\frac{1}{2}\right|+\left|\frac{\sum_{i=-n}^{n} \eta_{+}(i)-\sum_{i=-n}^{n} \eta_{t}(i)}{2 n+1}-\frac{1}{m}\right|
$$

Usando a equação 2.41 e $2 n+1=k m+l$ encontramos

$$
\left|\frac{\sum_{i=-n}^{n} \eta_{+}(i)}{2 n+1}-\left(\frac{1}{2}+\frac{1}{m}\right)\right| \leq \epsilon_{n}+\left|\frac{k}{2 n+1}-\frac{1}{m}\right| \leq \epsilon+\frac{1}{2 n+1}
$$

Definindo $\phi_{n}=\epsilon+\frac{1}{2 n+1}$. Vemos que $\phi_{n} \downarrow 0$ quando $n \rightarrow \infty$, pelo lema 7 encontramos que

$$
\frac{\sum_{i=-n}^{n} \eta_{+}(i)}{2 n+1} \stackrel{\text { q.c }}{\rightarrow} \frac{1}{2}+\frac{1}{m}>\frac{1}{2}
$$

Pela parte b do teorema 2 temos que a velocidade assintótica de uma partícula qualquer é

$$
\frac{1}{(1 / 2)+(1 / m)}-1=1-\frac{4}{m+2} \rightarrow 1 \text { quando } m \rightarrow \infty
$$

Isto prova o teorema.

\subsection{Estimativa do fluxo do AC 184.}

O fluxo do AC 184 é dado por

$$
\theta(\rho, t)=\nu_{\rho}\left(\eta_{0} \in \mathcal{B}_{2 t+2}: F^{t} \eta_{0}=10\right)
$$

Utilizando a relação entre o AC 184 e o modelo de crescimento de superfícies podem-se construir passeios aleatórios, e mediante a aproximação de Stirling no espírito dos cálculos feitos por Feller[13], pode-se fazer uma estimativa para o fluxo de AC 184.

Fluxo para o autômato celular 184

$$
\theta(\rho, t) \cong \begin{cases}\rho\left(1-\frac{[4 \rho(1-\rho)]^{t}}{\sqrt{\pi t}}\right) & \text { se } 0<\rho<\frac{1}{2} \\ (1-\rho)\left(1-\frac{[4 \rho(1-\rho)]^{t}}{\sqrt{\pi t}}\right) & \text { se } \frac{1}{2} \leq \rho<1\end{cases}
$$


Prova. Da definição, o fluxo pode-se expressar como

$$
\theta(\rho, t)=1-\rho-\nu_{\rho}(00)
$$

Onde $00=\left\{\eta_{0} \in \mathcal{B}_{2 t+2}: F^{t} \eta_{0}=00\right\}$. Podemos calcular

$$
\nu_{\rho}(00)=\sum_{\Gamma} \nu_{\rho}\left(\eta_{0} \in \mathcal{B}_{2 t+2}:\left(\eta_{0}(1), \eta_{0}(2) \ldots \ldots, \eta_{0}(2 t+2)=\left(a_{1}, a_{2} \ldots ., a_{2 t+2}\right)\right)\right.
$$

Onde $a_{k} \in\{0,1\} . \Gamma=\left\{\eta_{0} \in \mathcal{B}_{2 t+2}: F^{t} \eta_{0}=00\right\}$ é o conjunto de todas as configurações iniciais tal que $2 \sum_{i=1}^{k} \eta_{0}(i)<k$, para $1 \leq k \leq 2 t+2$. Equivalentemente, $\Gamma$ no modelo de crescimento de superfícies é o conjunto de trajetórias de um passeio aleatório que começa na origem no tempo $t=0$ e que a cada passo avança uma unidade à direita com probabilidade $1-\rho$ e a uma unidade à esquerda com probabilidade $\rho$. Terminando seu passeio no tempo $t$ no ponto $2 x$ e tal que se cumpre a seguinte propriedade

$$
\sum_{i=1}^{k} \zeta(i)>0, \text { para } 1 \leq k \leq 2 t+2
$$

Usando o Teorema do Votante (ver Feller [13]), o número de trajetórias deste passeio aleatório é $\frac{x}{t+1}\left(\begin{array}{c}2 t+2 \\ t+1-x\end{array}\right)$. Onde o número de zeros é $(t+1+x)$ e o número de uns é $(t+1-x)$. Portanto,

$$
\theta(\rho, t)=1-\rho-\sum_{x=1}^{t+1} \frac{x}{t+1}\left(\begin{array}{c}
2 t+2 \\
t+1-x
\end{array}\right) \rho^{t+1-x}(1-\rho)^{t+1+x}
$$

Usando a fórmula de Stirling $n \approx e^{-n} n^{n} \sqrt{2 \pi n}$ encontramos a equação (2.45).

Observação. No ponto crítico, $\rho=\frac{1}{2}$ para o modelo AC 184 encontramos

$$
\theta\left(\frac{1}{2}, t\right) \cong \rho\left(1-\frac{1}{\sqrt{\pi t}}\right)
$$

Então, a medida $\nu_{1 / 2}(00)$ se aproxima ao seu valor estacionário como $t^{-1 / 2}$. Vemos que longe do ponto crítico a aproximação é exponencial.

Teorema 4. Seja $\left\{\eta_{t}, t \in \mathbb{N}\right\}$ o autômato celular $184 \mathrm{com}$ distribuição inicial a medida produto de Bernoulli de densidade $\rho=\frac{1}{2}, \prod_{i \in \mathbb{Z}}\left\{\nu_{1 / 2}\left(\eta_{0}(i)=s\right)\right\}$ com $s \in\{0,1\}$. Com marginais iguais a $\nu_{1 / 2}\left(\eta_{0}(i)=1\right)=1-\nu_{1 / 2}\left(\eta_{0}(i)=0\right)=\frac{1}{2}$. Seja $\mu_{t}$ a distribuição de $\eta_{t}$. Então, $\mu_{t}$ converge para $\frac{1}{2}\left(\delta_{p}+\delta_{i}\right)$ quando $t \rightarrow \infty$ com decaimento da forma $t^{-\frac{1}{2}}$.

Onde $\delta_{p}$ é a medida concentrada na configuração que tem partículas com velocidade 1 nos números pares e buracos nos números ímpares de $\mathbb{Z}$ e $\delta_{i}$ é a medida concentrada na configuração que tem partículas nos números ímpares e buracos nos números pares de $\mathbb{Z}$. 
Não daremos a prova porque existe uma versão geral deste teorema para todo $\rho \in[0,1]$. Este foi provado por Belitsky e Ferrari [6].

Observação. Da estimativa do fluxo podemos garantir que este teorema cumpre-se. Vemos que para $\rho=1 / 2$ o fluxo assintótico é $\rho$ e o fluxo se aproxima deste valor segundo a taxa polinomial $t^{-1 / 2}$. Podemos também comprovar que a velocidade assintótica quando $t \rightarrow \infty$ é 1 (teorema 3 ). 


\section{Capítulo 3}

\section{Modelo de Fukui e Ishibashi}

Este modelo foi introduzido pelos físicos japoneses Fukui e Ishibashi, daí o nome do modelo. Este modelo generaliza o autômato celular 184. A dinâmica é a mesma só que aqui temos partículas que se podem mover a velocidades maiores que 1 . As partículas podem ter velocidades desde 0 até um máximo que chamaremos $v_{\max }$. As propriedades globais deste modelo, como os valores assintóticos, são as mesmas do autômato celular 184.

\subsection{Definição do Modelo}

O modelo de Fukui e Ishibashi, denotado por FI, tem dois parâmetros: a densidade $\rho$ de partículas e a velocidade máxima permitida $v_{m a x}$, que é um número inteiro positivo e finito.

O modelo FI tem a seguinte regra de evolução: Ao tempo $t=0$ partículas são distribuídas nos sítios de $\mathbb{Z}$, ao mais uma partícula por sítio. Todas as partículas no momento inicial de tempo têm velocidade zero. Segundo a dinâmica, elas irão aumentando sua velocidade em função do tamanho do gap até a próxima partícula à direita. Se $x(i, t)$ denota a posição da $i$-ésima partícula no instante $t$, a posição da próxima partícula à direita será $x(i+1, t)$. Denotamos por $g a p(i, i+1)=x(i+1, t)-x(i, t)-1$ o gap entre as partículas $i$ e $i+1$. As partículas atualizam suas posições simultaneamente segundo a regra:

$$
x(i, t+1)=x(i, t)+v(i, t) \quad \forall i \in \mathbb{Z}
$$

onde

$$
v(i, t)=\min \left(\operatorname{gap}(i, i+1), v_{\max }\right)
$$

é a velocidade da $i$-ésima partícula no instante $t$. Quando $v_{\max }=1$, este modelo é equivalente ao autômato celular 184 .

Em cada instante de tempo uma partícula avança deterministicamente um passo de tamanho $v_{\text {max }}$ se o gap entre esta e a próxima partícula à direita é maior que $v_{\text {max }}$, em caso contrário, está avançará um passo de tamanho do gap. 
O modelo de Fukui e Ishibashi com velocidade máxima igual a $v_{\max }:=m$ é equivalente ao autômato celular seguinte. Seja $\left\{\eta_{t}\right\}_{t \in \mathbb{N}}$ o autômato em todos os tempos e $\eta_{t}$ a configuração do autômato no tempo $t ; \eta(i, t)$ será o valor do autômato no ponto $i$ no instante $t$. Da mesma forma que o AC $184 \eta(i, t)=1$ representa uma partícula no sítio $i$ e $\eta(i, t)=0$ representa um buraco no sítio $i \in \mathbb{Z}$.

Seja $s: \mathbb{Z} \times \mathbb{N} \longmapsto\{0,1\}$ que satisfaz a equação

$$
\left.s(i, t+1)=f_{m}(s(i-m, t), s(i-m+1), t), \ldots, s(i+1, t)\right)
$$

para todo $i \in \mathbb{Z}$ e todo $t \in \mathbb{N}$. $s(i, t)$ representa o estado do sítio $i$ no tempo $t$ e a função $f_{m}:\{0,1\}^{m+2} \longmapsto\{0,1\}$ é a regra de evolução do AC. O número inteiro positivo $m$ é o rádio à esquerda e 1 é o rádio à direita do ponto $i$.

Definimos a função global $F:\{0,1\}^{\mathbb{Z}} \longmapsto\{0,1\}^{\mathbb{Z}}$ tal que

$$
\left.\left[F\left(\eta_{t+1}\right)\right](i)=f_{m}(\eta(i-m, t), \eta(i-m+1, t), \ldots, \eta(i+1), t)\right)
$$

para toda configuração $\eta_{t}$.

A fórmula explícita para a regra local $f_{m}$ é

$$
\begin{aligned}
& \left.f_{m}(\eta(i-m, t), \eta(i-m+1, t), \ldots, \eta(i+1), t)\right) \\
& \quad=\eta(i, t)-\min \{\eta(i, t), 1-\eta(i+1, t)\} \\
& \quad+\min \{\max [\eta(i-m, t), \eta(i-m+1, t \ldots \ldots, \eta(i-1, t)], 1-\eta(i, t))\}
\end{aligned}
$$

Podemos imediatamente perceber que se um sítio $i$ esta vazio no tempo $t$, então, no tempo $t+1$ este pode ser ocupado por uma partícula chegando da esquerda, mas não de um sítio detrás de $i-m$. Similarmente, se um sítio $i$ está ocupado no tempo $t$, este sítio ficará vazio no tempo $t+1$ se, somente se, o sítio $i+1$ estiver vazio.

Em geral, depois de $t$ iterações deste autômato o estado de um sítio $\eta(i, t)$ depende do bloco finito de configurações do estado inicial

$$
\mathcal{B}_{m t+t+1}=\{\eta:(\eta(i-m t, 0), \eta(i-m t+1,0), \ldots ., \eta(i, t), \eta(i+t, 0))\}
$$

mas não de qualquer outros sítios na configuração inicial. Similarmente, um bloco de tamanho $k$ igual a $(\eta(i, t), \eta(i+1, t), \ldots, \eta(i+k-1, t))$ depende do bloco finito da configuração inicial seguinte

$$
\mathcal{B}_{m t+t+k}=\{\eta:(\eta(i-m t, 0), \eta(i-m t+1,0), \ldots, \eta(i+k-1+t, 0))\}
$$

\subsection{Generalização do Modelo de Crescimento de Su- perfícies}

Este modelo denotado por GCS é um processo a tempo discreto com valores em $\mathcal{R}$. Onde $\mathcal{R}$ é o espaço de funções contínuas de $\mathbb{R}$ em $\mathbb{R}$ com inclinação de $45^{\circ}$ ou $270^{\circ}+$ 
$\arctan \left(\frac{1}{m}\right)$ entre qualquer duas abscissas inteiras. O valor que atinge em cada abscissa inteira é também inteiro e $m$ é um número inteiro positivo e finito.

A dinâmica do GCS será definida pelo operador $S: \mathcal{R} \rightarrow \mathcal{R}$ tal que $f_{t+1}()=.S f_{t}$. Se $f_{t}(.) \in \mathcal{R}$ representa a configuração de $\mathcal{R}$, no instante de tempo $t, t \in \mathbb{N}=\{0,1,2 \ldots\}$, então, $f_{t+1}(.) \in \mathcal{R}$ é definido por

$$
f_{t+1}(.):=\left\{\begin{array}{cl}
f(i)+1+m, & \forall i \in\{i, \ldots, m-1\} \text { se } f(i)=f(i+1)-m=f(i-1)-m \\
f(i), & \text { caso contrário }
\end{array}\right.
$$

para todo $i \in \mathbb{Z}$.

Relação entre Modelo FI e o Modelo GCS.

Consideremos os modelos FI e GCS e definamos

$T_{G C S, F I}: \mathcal{R} \rightarrow\{0,1\}^{\mathbb{Z}}$ por

$$
\left(T_{S G, 184} f\right)(i)= \begin{cases}0 & \text { se } f(i)-f(i-1)=1 \\ 1 & \text { se } f(i)-f(i-1)=-m\end{cases}
$$

para todo $i \in \mathbb{Z}$.

Definamos $\xi(i):=f(i)-f(i-1)$ para todo $i \in \mathbb{Z}$. Com isto, usando a relação acima teremos

$$
\xi(i)=1-(m+1) \eta(i)= \begin{cases}1 & \text { se } \eta(i)=0 \\ -m & \text { se } \eta(i)=1\end{cases}
$$

Dada a configuração inicial do processo de Fukui-Ishibashi $\eta_{0}$. Podemos representar $\xi$ como o retrato de uma superfície que por cada 0 aumenta seu valor em um e por cada 1 diminui seu valor em $m$.

\subsection{Resultados}

Proposição 1. Consideremos que $\left\{\eta_{t}, t \in \mathbb{N}\right\}$ represente o Modelo de Fukui-Ishibashi com velocidade máxima $v_{\max }:=m$ e distribuição inicial $\eta_{0}$.

A condição necessária e suficiente para que uma partícula na origem entre em fluxo livre é

$$
\exists k\left(\eta_{0}\right): \quad(m+1) \sum_{i=1}^{k} \eta(i)<k, \quad \forall k \geq k\left(\eta_{0}\right)
$$

Prova. Usaremos a relação do autômato de Fukui-Ishibashi com o modelo de crescimento de superfícies.

Definamos $\xi(i):=f(i)-f(i-1)$ para todo $i \in \mathbb{Z}$. Com isto, usando a relação acima teremos

$$
\xi(i)=1-(m+1) \eta(i)= \begin{cases}1 & \text { se } \eta(i)=0 \\ -m & \text { se } \eta(i)=1\end{cases}
$$


Com isto e da equação (3.8) obtemos a equivalência

$$
\sum_{i=1}^{k} \xi(i)>0 \quad \forall k>k\left(\eta_{0}\right) \in \mathbb{N}
$$

Provemos primeiro que se existe um $r:=k\left(\eta_{0}\right)$ tal que se cumpre a equação 3.10, então, a partícula na origem é livre a partir de um tempo aleatório $t_{0}=t_{0}\left(\eta_{0}\right) \leq r$.

Consideremos o bloco finito $\mathcal{B}_{m t+m+t+1}$ de tamanho $n=m t+m+t+1$ da configuração inicial $\eta_{0} \in \Omega=\{0,1\}^{\mathbb{Z}}$, que começa no ponto $r=r\left(\eta_{0}\right)$

$$
\begin{aligned}
& \mathcal{B}_{m t+m+t+1}= \\
& \quad\left\{\eta \in \Omega:(\eta(r+1,0), \eta(r+2,0), \ldots, \eta(r+m t+m+t+1,0))=\left(a_{1}, \ldots, a_{m t+m+t+1}\right)\right\}
\end{aligned}
$$

Logo teremos que

$$
\sum_{i=r+1}^{r+k} \xi(i)>0, \quad \text { para } 1 \leq k \leq(m+1)(t+1)
$$

Da representação gráfica do processo GCS vemos que isto acontece se, somente se, no intervalo $[r+1, r+(m+1)(t+1)] \cap \mathbb{Z}$ para cada $k$ tal que $1 \leq k \leq(m+1)(t+1)$ o número de anti-bonds é maior que o número de bonds. Ademais, necessariamente

$$
\eta_{0}(r+1)=\eta_{0}(r+2)=\ldots=\eta_{0}(r+m+1)=0_{m+1}:=\underbrace{000 \ldots \ldots 00}_{m+1 \text { zeros }}
$$

Passando a configuração inicial ao seu correspondente processo de aniquilação teremos pelo lema 6 que todos os bonds que existem serão aniquilados com probabilidade 1 . Como resultado da evolução teremos que depois de $n$ interações encontraremos só o bloco $0_{m+1}$, isto é, $\eta_{n}(r+n+1) \eta_{n}(r+n+2) \ldots . \eta_{r+n}=0_{m+1}$. Logo, temos

$$
\mathrm{h}^{n}\left(\eta_{0}(r+1), \eta_{0}(r+2) \ldots, \eta_{0}(r+n)\right)=0_{m+1}
$$

Então, a partir do momento $t_{0}\left(\eta_{0}\right) \leq r$ a partícula que está inicialmente na origem passara ao estado de fluxo livre.

Provemos a volta. Se a partícula na origem está livre para sempre, então, existe um $t_{0}\left(\eta_{0}\right)$ tal que a partir deste tempo a partícula $C_{0}$ será livre. Teremos que a partir do ponto $x_{0}(t)$ (que é a posição desta partícula no tempo $t_{0}$ ) a seguinte relação cumpre-se

$$
\exists k\left(\eta_{0}\right)=t_{0}\left(\eta_{0}\right): 2 \sum_{i=x_{0}(t)+1}^{x_{0}(t)+k} \eta(i)<k, \quad \forall k \geq k\left(\eta_{0}\right)
$$

A partir do ponto $x_{0}\left(t_{0}\right)$ à direita todas as partículas são livres. Portanto, existe ao menos uma partícula $C_{j}$ na posição $x_{j}\left(t_{0}\right)=x_{j}+m t_{0}$ que é livre desde o início. Esta 
partícula na configuração inicial tinha a posição $x_{j}$. Portanto, se a partícula na origem é livre, então, existe um $k\left(\eta_{0}\right)=x_{j}$ tal que,

$$
F^{t_{0}}\left(\eta_{0}\left(x_{j}+1\right), \eta_{0}\left(x_{j}+2\right) \ldots ., \eta_{0}\left(x_{j}+m t_{0}+m+t_{0}+1\right)\right)=0_{m+1}
$$

Vemos que isto acontece, se somente se,

$$
\eta_{t_{0}}\left(x_{j}+m t_{0}+1\right) \eta_{t_{0}}\left(x_{j}+m t_{0}+2\right) \ldots . \eta_{t_{0}}\left(x_{j}+m t_{0}+m+1\right)=0_{m+1}
$$

Usando a transformação no processo de aniquilação para este sub-bloco teríamos $0 A$, onde $0 A$ corresponde à configuração finita $10_{m+1} 1$ e $0 B$ corresponde à configuração 11 . Desde que as partículas de classe-A movem-se à direita a velocidade 1 , isto significa que no instante $t=0$ devíamos de ter $0 A$ ou usando o modelo de Fukui-Ishibashi

$$
\eta_{0}\left(x_{j}+1\right) \eta_{0}\left(x_{j}+2\right) \ldots \eta_{0}\left(x_{j}+m+1\right)=0_{m+1}
$$

O que significa que a partícula de classe-A viaja de $i=x_{j}+m t_{0}+1$ até $i=x_{j}+m t_{0}+m+1$ em $t_{0}$ unidades de tempo. Esta partícula pode viajar esta distância sem ser aniquilada se, somente se, não colide com uma partícula de classe-B que se move em sentido contrário. Isto pode acontecer se todas as partículas de classe-B são aniquiladas antes de encontrarse com esta partícula de classe- $\mathrm{A}$, isto é , se para cada $m t_{0}+1 \leq k \leq m t_{0}+m+t_{0}+1$ o número de partículas de classe-B (bonds) dentro do sub-bloco $\eta_{0}\left(x_{j}+m t_{0}+1\right), \eta_{0}\left(x_{j}+\right.$ $\left.m t_{0}+2\right) \ldots ., \eta_{0}\left(x_{j}+m t_{0}++m+t_{0}+1\right)$ é menor que o número de partículas de classe-A (anti-bonds). Usando a notação de zeros e uns temos

$$
\begin{gathered}
F^{t_{0}}\left(\eta_{0}\left(x_{j}+1\right), \eta_{0}\left(x_{j}+2\right) \ldots ., \eta_{0}\left(x_{j}+m t_{0}+m+t_{0}+1\right)\right)=0_{m+1} \Longrightarrow \\
\sum_{i=x_{j}+1}^{k} \xi(i)>0 \text { para } 1 \leq k \leq(m+1)\left(t_{0}+1\right) .
\end{gathered}
$$

Isto prova a proposição.

Teorema 1. Consideremos $\left\{\eta_{t}, t \in \mathbb{N}\right\}$ o Modelo de Fukui-Ishibashi com velocidade máxima $v_{\max }:=m$ e distribuição inicial a medida produto de Bernoulli de densidade $\rho \in[0,1]$. Com marginais iguais a $\nu_{\rho}\left(\eta_{0}(i)=1\right)=1-\nu_{\rho}\left(\eta_{0}(i)=0\right)=\rho$.

Se $0<\rho<\frac{1}{1+v_{\max }}$, então, as partículas entram em estado de fluxo livre com probabilidade 1.

Prova Como $0<\rho<\frac{1}{1+v_{\max }}$, pela lei dos grande números com probabilidade 1 cumpre-se

$$
\exists N\left(\eta_{0}\right):\left|\frac{1}{n} \sum_{i=1}^{n} \eta(i)-\rho\right|<\epsilon, \forall n>\mathbb{N}\left(\eta_{0}\right), \forall \epsilon>0
$$

Trabalhando um lado da desigualdade temos 


$$
\frac{1}{n} \sum_{i=1}^{n} \eta(i)<(\rho+\epsilon)
$$

Como $\epsilon>0$ é arbitrário escolhemos $\epsilon$ tal que $\epsilon<\frac{1}{1+m}-\rho$. Com isto, conseguimos

$$
\exists N\left(\eta_{0}\right):(m+1) \sum_{i=1}^{k} \eta(i)<k, \quad \forall k \geq N\left(\eta_{0}\right)
$$

Usando a proposição 1 o resultado é direto. Portanto, teremos fluxo livre.

Teorema 2. Consideremos $\left\{\eta_{t}, t \in \mathbb{N}\right\}$ o Modelo de Fukui-Ishibashi com velocidade máxima $v_{\max }:=m$ e distribuição inicial a medida produto de Bernoulli de densidade $\rho \in[0,1]$. Com marginais iguais a $\nu_{\rho}\left(\eta_{0}(i)=1\right)=1-\nu_{\rho}\left(\eta_{0}(i)=0\right)=\rho$.

a. Se $0<\rho<\frac{1}{1+v_{\max }}$, então, a velocidade assintótica das partículas será $m$.

b. Se $\frac{1}{1+v_{\max }}<\rho<1$, então, a velocidade assintótica das partículas será $\frac{1}{\rho}-1$.

c. O fluxo de $\theta$ é dado por

$$
\theta(\rho):= \begin{cases}v_{\max } \rho & \text { se } 0<\rho<\frac{1}{1+v_{\max }} \\ 1-\rho & \text { se } \frac{1}{1+v_{\max }} \leq \rho<1\end{cases}
$$

Prova a. Pela parte a do teorema 1 temos que se $0<\rho<\frac{1}{m+1}$ qualquer partícula entra em fluxo livre. Consideremos a partícula $C_{n}$, portanto, existe um tempo $t_{n}=t_{n}\left(\eta_{0}\right)$ a partir do qual a velocidade desta partícula será $m$.

Calculando a velocidade assintótica teremos.

$$
v=\lim _{t \rightarrow \infty} \frac{1}{t} L\left(\eta_{0}, x_{n}, t\right)
$$

Para qualquer partícula que inicialmente se encontra no ponto $x_{n} \in \mathbb{Z}$ a distância total $L\left(\eta_{0}, x_{n}, t\right)$ que avança durante o tempo $t$ é o número de buracos que $C_{n}$ encontra no seu caminho durante este tempo. Teremos logo,

$$
v=\lim _{t \rightarrow \infty}\left\{\frac{1}{t} \sum_{s=0}^{t_{n}} L\left(\eta_{0}, x_{n}, s\right)+\frac{1}{t} \sum_{s=t_{n}+1}^{t-1} L\left(\eta_{0}, x_{n}, s\right)\right\}=m
$$

A primeira soma é zero. Na segunda soma temos que para $s>t_{n}$ a partícula $C_{n}$ está livre, portanto, em cada passo encontrará $m$ buracos. Logo, temos que a velocidade assintótica é $v=v_{\max }=m$.

Parte b: Se $\frac{1}{1+m}<\rho<1$ calculemos a velocidade assintótica da partícula $C_{n}$. 


$$
v=\lim _{t \rightarrow \infty} \frac{1}{t} L\left(\eta_{0}, x_{n}, t\right)
$$

$L\left(\eta_{0}, x_{n}, t\right)$ é o número de buracos que $C_{n}$ encontra no seu caminho. Denotemos por $b_{n, t}$ a posição original (no instante $t=0$ ) na configuração $\eta_{0}$ do último buraco que a partícula $C_{n}$ encontrará em seu caminho durante o tempo $t$. Então, no bloco $\mathcal{B}_{\left[x_{n}, b_{n, t}\right]}$ há exatamente $L\left(\eta_{0}, x_{n}, t\right)$ buracos e podemos estabelecer a equação

$$
L\left(\eta_{0}, x_{n}, t\right)=\left(b_{n, t}-x_{n}\right) \cdot\left(1-\rho_{t}\right)
$$

Além disso o número de partículas neste bloco, denotado por $N_{t}$, será

$$
N_{t}=\left(b_{n, t}-x_{n}\right) \cdot \rho_{t}
$$

Onde $\rho_{t}$ é a densidade das partículas no bloco considerado.

Temos para a velocidade média

$$
\bar{V}\left(\eta_{0}, x_{n}, t\right)=\frac{1}{t} L\left(\eta_{0}, x_{n}, t\right)=\frac{N_{t}}{t} \times \frac{1-\rho_{t}}{\rho_{t}}
$$

Tomando limite temos

$$
v=\frac{1-\rho}{\rho} \times \lim _{t \rightarrow \infty} \frac{N_{t}}{t}
$$

Clamamos que $\lim _{t \rightarrow \infty} \frac{N_{t}}{t}=1$. Para calcular este limite usamos a propriedade: Em alta densidade as partículas se comportam como se fosse um autômato celular 184, isto acontece devido ao fato das partículas não poderem-se mover em mais de um espaço por vez. Portanto, para um buraco colocado no ponto $b_{n, t}$ que pertence ao mesmo bloco temos que $N_{t}$ é a quantidade de partículas que encontra no seu caminho, mas a partir de certo tempo este buraco avançará livremente e, portanto, o limite deve de ser 1 . Logo, temos que a velocidade assintótica é $v=\frac{1}{\rho}-1$.

Parte c. O fluxo assintótico é definido por $\theta(\rho)=\rho v$, onde $v$ é a velocidade assintótica das partículas. Da parte a e b sabemos que as velocidades assintóticas são

$$
v= \begin{cases}v_{\max } & \text { se } 0<\rho<\frac{1}{1+m} \\ \frac{1-\rho}{\rho} & \text { se } \frac{1}{1+m} \leq \rho<1\end{cases}
$$

Com isto a prova está concluída. 


\section{Capítulo 4}

\section{Autômato Celular com Aceleração Aleatória}

Neste capítulo, estudaremos um modelo simplificado do autômato celular proposto por Schadschneider e Schreckenberg [26] denominado autômato com regras slow-to-star. Introduzimos o Autômato Celular Probabilista denominado Autômato Celular com Aceleração Aleatória, denotado por ACAA. Este autômato tem dinâmica aleatória. Teremos partículas com velocidade 0 , denominadas partículas paradas e partículas com velocidade 1, denominadas partículas aceleradas. As partículas paradas esperam um tempo aleatório com distribuição geométrica de parâmetro $p$ para acelerar e ganhar velocidade 1 . A probabilidade $p$ será denominada parâmetro de aceleração e junto com a densidade inicial de distribuição das partículas serão os dois parâmetros do ACAA.

As partículas com velocidade 1 se movem determinísticamente como se sua regra de evolução fosse o do Autômato Celular 184. Todas as partículas aceleradas se movem à velocidade 1 para sempre ou, em caso contrário, se deterão se encontrarem alguma partícula parada na sua frente que bloqueie seu movimento. Neste caso, sua velocidade voltará para 0 e ficará bloqueada até a partícula ou as partículas que bloqueiam seu caminho tenham acelerado. A partir deste momento, poderá acelerar com probabilidade $p$.

\subsection{Definição do modelo}

Uma configuração sobre $\mathcal{Q}=\{0,1, e\}$ é uma função de $\mathbb{Z}$ em $\mathcal{Q}$. Denotemos por $\Omega=\mathcal{Q}^{\mathbb{Z}}$ o espaço de configurações sobre $\mathcal{Q}$. Se $\eta \in \Omega$ será chamado de configuração e $\eta(i), \forall i \in \mathbb{Z}$, será o valor da configuração $\eta$ no ponto $i$.

Como o autômato evolui no tempo, $\left\{\eta_{t}\right\}_{t \in \mathbb{N}}$, indica o autômato em todos os tempos e $\eta_{t}$ será a configuração do autômato no tempo $t ; \eta(i, t)$ será o valor do autômato no ponto $i$ no instante $t$.

O Autòmato Celular com Aceleração Aleatória tem três estados e, 0, 1. Onde e representa $u$ m sítio vazio, 0 representa uma partícula parada e 1 representa uma partícula acelerada. O autômato tem a seguinte regra de evolução: Ao tempo $t=0$ partículas com 
velocidade 0 são distribuídas nos sítios de $\mathbb{Z}$, ao mais uma partícula por sítio. Esta distribuição será feita usando a medida produto de Bernoulli $\nu_{\rho}$ de densidade $\rho$, qual distribui uma partícula em cada sítio independentemente com densidade $\rho \in[0,1]$.

Consideremos para o ACAA o espaço de probabilidade $\left(\{0, e\}^{\mathbb{Z}}, \mathcal{B}, \nu_{\rho}\right)$, onde $\mathcal{B}$ é a sigma álgebra gerada pelos conjuntos cilíndricos de $\{0, e\}^{\mathbb{Z}}$ e $\nu_{\rho}$ é a medida produto de Bernoulli que colocamos no início do processo. O ACAA modela o movimento de partículas para a direita na reta infinita $\mathbb{Z}$, onde a aleatoriedade do modelo surge de dois processos : um é introduzida pela condição inicial e o outro é introduzida pela dinâmica de movimento.

A dinâmica de evolução é a seguinte:

1. Se $\eta_{t}(i) \eta_{t}(i+1)=0 e$, então no tempo $t+1$ a partícula de velocidade 0 muda para velocidade $1 \mathrm{e}$, simultaneamente, avança um espaço à direita com probabilidade $p$. Passando ao estado $\eta_{t+1}(i) \eta_{t+1}(i+1)=e 1$.

2. Se $\eta_{t}(i) \eta_{t}(i+1)=1 e$, então no tempo $t+1$ a partícula de velocidade 1 avança um espaço à direita deterministicamente. Passando ao estado $\eta_{t+1}(i) \eta_{t+1}(i+1)=e 1$.

3. Se $\eta_{t}(i) \eta_{t}(i+1)=01$, então no tempo $t+1$ a partícula de velocidade 0 permanece com velocidade 0 e a partícula com velocidade 1 avança deterministicamente se tiver espaço livre. Passando ao estado $\eta_{t+1}(i) \eta_{t+1}(i+1)=0 *$.

4. Se $\eta_{t}(i) \eta_{t}(i+1)=10$, então no tempo $t+1$ a partícula de velocidade 1 muda sua velocidade para $0 \mathrm{com}$ probabilidade 1 . Passando ao estado $\eta_{t+1}(i) \eta_{t+1}(i+1)=0 *$.

5. Se temos partículas bloqueadas, estas esperam até se desbloquearem para usar as regras anteriores.

Onde $*$ indica um estado indeterminado.

\subsection{Resultados}

O seguinte teorema mostra a transição de fase no modelo.

Teorema 1. Seja $\left\{\eta_{t}, t \in \mathbb{N}\right\}$ o ACAA com parâmetro de aceleração $p$ e distribuição inicial a medida produto de Bernoulli de densidade $\rho \in[0,1], \prod_{i \in \mathbb{Z}}\left\{\nu_{\rho}\left(\eta_{0}(i)=s\right)\right\}$ com $s \in\{0, e\}$. Com marginais iguais a $\nu_{\rho}\left(\eta_{0}(i)=0\right)=1-\nu_{\rho}\left(\eta_{0}(i)=e\right)=\rho$. Se definimos

$$
\rho_{c}=\sup \{\rho: \theta(\rho, p)=\rho\}
$$

Então,

$$
\frac{p}{1+p} \leq \rho_{c} \leq \frac{1}{2}
$$

Prova. A cota superior $1 / 2$ decorre da seguinte propriedade: O ACAA no limite $p \rightarrow 1$ é equivalente ao autômato celular 184. As dinâmicas são as mesmas quando $p=1$, nesse sentido são equivalentes. 
Para provar a cota inferior usaremos a técnica usada por Gray e Griffeath[16] ${ }^{1}$

Assumiremos que $\rho>\rho_{c}$, e mostraremos que $\rho \geq \frac{p}{1+p}$. Portanto, da definição de $\rho_{c}$ (equação 4.1) teremos a prova do teorema.

Condicionado sobre o evento que a partícula $C_{0}$ esteja na origem no tempo inicial $t=0$, seja $X_{t}$ a posição dessa partícula no tempo $t \geq 0$ e seja $Y_{t}$ a posição da partícula $C_{1}$ (partícula mais próxima de $C_{0}$ ) à direita de $C_{0}$. Pela conservação de partículas teremos que $\mathbb{E}\left(Y_{t}-X_{t}\right)=\frac{1}{\rho}$ para todo $t \geq 0$. Definimos os seguintes eventos para $0 \leq s<t$ :

$$
\begin{gathered}
A_{t}=\left\{Y_{t}-X_{t} \leq 2\right\} \\
B_{s, t}=\left\{Y_{s}-X_{s}=2 \text { e } Y_{u}-X_{u}>2 \text { para } s<u \leq t\right\} \\
C_{t}=\left\{Y_{u}-X_{u}>2 \text { para } 0 \leq u \leq t\right\}
\end{gathered}
$$

Usando a conservação das partículas temos

$$
\frac{1}{\rho}=\mathbb{E}\left\{\left(Y_{t}-X_{t} \mid A_{t}\right) \mathbb{P}\left(A_{t}\right)\right\}+\sum_{s=0}^{t-1}\left\{\mathbb{E}\left(Y_{t}-X_{t} \mid B_{s, t}\right) \mathbb{P}\left(B_{s, t}\right)\right\}+\mathbb{E}\left\{\left(Y_{t}-X_{t} \mid C_{t}\right) \mathbb{P}\left(C_{t}\right)\right\}
$$

Sob a asunção de $\rho>\rho_{c}$, temos que $\lim _{t \rightarrow \infty} \mathbb{P}\left(C_{t}\right)=0$, ademais $\mathbb{E}\left\{\left(Y_{t}-X_{t} \mid A_{t}\right)\right\} \leq 2$. Tomando limite quando $t \rightarrow \infty$, temos

$$
\frac{1}{\rho} \leq \lim _{t \rightarrow \infty}\left[2 \mathbb{P}\left(A_{t}\right)+\sum_{s=0}^{t}\left\{\mathbb{E}\left(Y_{t}-X_{t} \mid B_{s, t}\right) \mathbb{P}\left(B_{s, t}\right)\right\}\right]
$$

Ademais temos que

$$
\frac{p}{1+p}<\frac{1}{2} \Leftrightarrow 2<\frac{1+p}{p}
$$

Desde que $2<\frac{1+p}{p}$ para $p>0$, para conseguir a cota inferior necessitamos provar que

$$
\mathbb{E}\left(Y_{t}-X_{t} \mid B_{s, t}\right) \leq \frac{1+p}{p} \text { para todo } 0 \leq s<t
$$

Realmente provaremos algo mais que isso

$$
\mathbb{E}\left(Y_{t}-X_{t} \mid B_{s, t}, Y_{t}-Y_{s}\right) \leq \frac{1+p}{p} \text { para todo } 0 \leq s<t
$$

Dado o evento $B_{s, t}$ teremos que a partir do tempo $s+1$ as partículas $C_{0}$ e $C_{1}$ estão separadas ao menos dois espaços. A partícula $C_{1}$ no instante $s+1$ tem velocidade 1 e $C_{0}$ está parada. Seja $K=(t-s)-\left(Y_{t}-Y_{s}\right)$. Se $B_{s, t}$ ocorre, então, a partícula $C_{0}$ não deverá mover-se entre os tempos $s+1, \ldots, s+K$ porque teríamos que a distância $Y_{u}-X_{u}=2$ para algum $u$ no intervalo $(s, t]$, o que contradiz $B_{s, t}$. A partir do tempo $s+K$, a variável geométrica $T$, tempo de espera para que a partícula $C_{0}$, se mova é independente de $B_{s, t}$

\footnotetext{
${ }^{1}$ Este teorema foi sugerido por Griffeath numa conversa pessoal
} 
e $Y_{t}-Y_{s}$ e, ademais, temos que $Y_{t}-X_{t}=1+T$ onde $T$ é o número de lançamentos de uma moeda até sair a primeira cara começando a partir de tempo $s+K+1$.

Temos, geralmente, que se o evento $B_{s, t}$ ocorre, então, $Y_{t}-X_{t} \leq 1+T$. Como $\mathbb{E} T=\frac{1}{p}$ temos

$$
\mathbb{E}\left(Y_{t}-X_{t} \mid B_{s, t}, Y_{t}-Y_{s}\right) \leq 1+\frac{1}{p}=\frac{1+p}{p} \text { para todo } 0 \leq s<t
$$

Isto prova o teorema.

Corolário 1. Seja $\left\{\eta_{t}, t \in \mathbb{N}\right\}$ o ACAA com parâmetro de aceleração $p$ e distribuição inicial a medida produto de Bernoulli de densidade $\rho \in[0,1], \prod_{i \in \mathbb{Z}}\left\{\nu_{\rho}\left(\eta_{0}(i)=s\right)\right\}$ com $s \in\{0,1\}$. Com marginais iguais a $\nu_{\rho}\left(\eta_{0}(i)=0\right)=1-\nu_{\rho}\left(\eta_{0}(i)=e\right)=\rho$.

Se $0<\rho<\frac{p}{1+p}$, então, as partículas entram em fluxo livre com probabilidade 1 .

Prova. Direto da definição de densidade crítica e do resultado do teorema 1.

Teorema 2. Seja $\left\{\eta_{t}, t \in \mathbb{N}\right\}$ o ACAA com parâmetro de aceleração $p$ e distribuição inicial a medida produto de Bernoulli de densidade $\rho \in[0,1], \prod_{i \in \mathbb{Z}}\left\{\nu_{\rho}\left(\eta_{0}(i)=s\right)\right\}$ com $s \in\{0, e\}$. Com marginais iguais a $\nu_{\rho}\left(\eta_{0}(i)=0\right)=1-\nu_{\rho}\left(\eta_{0}(i)=e\right)=\rho$.
a. Se $0<\rho<\frac{p}{1+p}$, então, o fluxo assintótico é $\theta(\rho, p)=\rho$.
b. Se $\rho>\frac{1}{2}$, então, o fluxo assintótico é $\theta(\rho, p)=p(1-\rho)$.

\section{Prova}

Parte a. Pelo Corolário 1 temos que se $0<\rho<\frac{p}{1+p}$ qualquer partícula entra em fluxo livre. Consideremos a partícula $C_{n}$. Existe um tempo $t_{n}=t_{0}\left(\eta_{0}\right)$ a partir do qual a velocidade desta partícula será 1 .

Calculando a velocidade assintótica temos:

$$
v=\lim _{t \rightarrow \infty} \frac{1}{t} L\left(\eta_{0}, x_{n}, t\right)
$$

Para qualquer partícula que inicialmente se encontra no ponto $x_{n} \in \mathbb{Z}$, a distância total $L\left(\eta_{0}, x_{n}, t\right)$ que avança durante o tempo $t$ é o número de buracos que $C_{n}$ encontra no seu caminho durante este tempo. Esta quantidade pode ser expressa por

$$
L\left(\eta_{0}, x_{n}, t\right)=\sum_{s=0}^{t-1} 1\left\{V\left(C_{n}, s\right)=1\right\}
$$

onde $V\left(C_{n}, t\right)$ é a velocidade da partícula $C_{n}$ no instante $t$. Teremos logo

$$
v=\lim _{t \rightarrow \infty}\left\{\frac{1}{t} \sum_{s=0}^{t_{n}} L\left(\eta_{0}, x_{n}, s\right)+\frac{1}{t} \sum_{s=t_{n}+1}^{t-1} L\left(\eta_{0}, x_{n}, s\right)\right\}=1
$$


A primeira soma é zero. Na segunda soma temos que para $s>t_{n}$ a partícula $C_{n}$ está livre, portanto, em cada passo encontrará um buraco. Logo, temos que a velocidade assintótica é $v=1$.

Parte b. Se $\frac{1}{2}<\rho<1$ calculemos a velocidade assintótica da partícula $C_{n}$.

$$
v=\lim _{t \rightarrow \infty} \frac{1}{t} L\left(\eta_{0}, x_{n}, t\right)
$$

$L\left(\eta_{0}, x_{n}, t\right)$ é o número de buracos que $C_{n}$ encontra no seu caminho e é dada por

$$
L\left(\eta_{0}, x_{n}, t\right)=\sum_{s=0}^{t-1} 1\left\{V\left(C_{n}, s\right)=1\right\}
$$

Denotemos por $b_{n, t}$ a posição original (no instante $t=0$ ) na configuração $\eta_{0}$ do último buraco que a partícula $C_{n}$ encontrará em seu caminho durante o tempo $t$. Então, no bloco $\mathcal{B}_{\left[x_{n}, b_{n, t}\right]}$ há exatamente $L\left(\eta_{0}, x_{n}, t\right)$ buracos e podemos estabelecer a equação

$$
L\left(\eta_{0}, x_{n}, t\right)=\left(b_{n, t}-x_{n}\right) \cdot\left(1-\rho_{t}\right)
$$

Além disso o número de partículas neste bloco, denotado por $N_{t}$, será

$$
N_{t}=\left(b_{n, t}-x_{n}\right) \cdot \rho_{t}
$$

Onde $\rho_{t}$ é a densidade das partículas no bloco considerado.

Temos para a velocidade média

$$
\bar{V}\left(\eta_{0}, x_{n}, t\right)=\frac{1}{t} L\left(\eta_{0}, x_{n}, t\right)=\frac{N_{t}}{t} \times \frac{1-\rho}{\rho}
$$

Tomando limite temos

$$
v=\frac{1-\rho}{\rho} \times \lim _{t \rightarrow \infty} \frac{N_{t}}{t}
$$

Clamamos que $\lim _{t \rightarrow \infty} \frac{N_{t}}{t}=p$. Para calcular este limite usemos a seguinte propriedade: Durante o tempo $t$ o buraco que começo seu movimento na posição $b_{n, t}$ se encontrará com todas as partículas $N_{t}$ com taxa de uma partícula cada $\mathbb{E}(T)$ passos, onde $T$ é o tempo de espera para mudar de velocidade. Logo, temos que a velocidade assintótica e fluxo são dados por

$$
v=p\left(\frac{1}{\rho}-1\right) \text { e } \theta(\rho, p)=p(1-\rho)
$$

\subsection{Modelo Modificado do ACAA}

Introduzimos uma variante do autômato com aceleração probabilista. Nesta variante enfraquecemos a interação tipo exclusão permitindo que várias partículas ocupem o mesmo sítio. O autômato celular probabilista tem distribuição inicial a medida produto 
de Bernoulli de densidade $\rho$ e a dinâmica de evolução dada por: Cada partícula espera um tempo aleatório que tem distribuição geométrica de parâmetro $p$ para mover-se pela primeira vez. Após este tempo, as partículas movem-se com velocidade 1 para sempre ou, em caso contrário, se deterão (várias partículas podem ocupar o mesmo sítio) se encontrarem alguma partícula parada na sua frente que bloqueie seu movimento. Neste caso, as velocidades voltarão para 0 e as partículas ficarão bloqueadas até que a partícula ou as partículas que bloqueiam seus caminhos tenham partido. A partir deste instante, a partícula não bloqueada espera mais um tempo aleatório com distribuição geométrica de parâmetro $p$ para mover-se. Usaremos a construção gráfica para o caso contínuo feita no capítulo 5 para mapear este modelo em um sistema de filas discreto com um único servidor. Provamos que se $\rho<p$, a distância entre as partículas converge quase certamente à distância entre as saídas de um cliente numa fila discreta estacionária Geom $/$ Geom $/ 1 / \infty$ associada ao sistema.

Teorema 3. Seja $\left\{\eta_{t}, t \in \mathbb{N}\right\}$ o ACAA com parâmetro de aceleração $p$ e distribuição inicial a medida produto de Bernoulli de densidade $\rho \in[0,1], \prod_{i \in \mathbb{Z}}\left\{\nu_{\rho}\left(\eta_{0}(i)=s\right)\right\}$ com $s \in\{0, e\}$. Com marginais iguais a $\nu_{\rho}\left(\eta_{0}(i)=0\right)=1-\nu_{\rho}\left(\eta_{0}(i)=e\right)=\rho$. Considerando a propriedade de enfraquecimento da interação de exclusão. Se $\rho<p$, então,

$$
\lim _{t \rightarrow \infty}\left(x_{n}(t)-x_{n-1}(t)\right)=s_{n}-s_{n-1} \quad \text { quase certamente }
$$

Onde $s_{n}$ é o tempo de saída do $n$-ésimo cliente de uma fila discreta Geom $/$ Geom $/ 1 / \infty$.

Prova. A prova (versão discreta) é a mesma da que faremos no capítulo 5 para o caso contínuo.

\subsection{Relação do ACAA com um Sistema de Filas Dis- creto}

Conjectura 1. Seja $\left\{\eta_{t}, t \in \mathbb{N}\right\}$ o ACAA com parâmetro de aceleração $p$ e distribuição inicial a medida produto de Bernoulli de densidade $\rho \in[0,1], \prod_{i \in \mathbb{Z}}\left\{\nu_{\rho}\left(\eta_{0}(i)=s\right)\right\}$ com $s \in\{0, e\}$. Com marginais iguais a $\nu_{\rho}\left(\eta_{0}(i)=0\right)=1-\nu_{\rho}\left(\eta_{0}(i)=e\right)=\rho$.

Se $\rho<\frac{p}{p+1}$, então, a distância entra as partículas no autômato celular com aceleração aleatória converge quase certamente à distância entre as saídas de um cliente numa fila discreta estacionária Geom $/[$ Geom +1$] / 1 / \infty$ associada ao sistema. 


\section{Capítulo 5}

\section{Modelo Pontual de Trânsito em $\mathbb{R}$}

Neste capítulo, consideraremos um modelo de trânsito de partículas que é contínuo no tempo e no espaço, que denominaremos Modelo Pontual. No instante inicial distribuímos partículas em $\mathbb{R}$ segundo um processo pontual marcado. O processo pontual é um processo de Poisson de parâmetro $\lambda<1$ que indica as posições das partículas e as marcas são seqüências iid de distribuição exponencial de média 1, que definem os tempos de espera para que as partículas mudem de velocidade 0 para velocidade -1 . Cada partícula espera um tempo aleatório que tem distribuição exponencial para mover-se pela primeira vez. Após este tempo, as partículas movem-se com velocidade -1 para sempre ou, em caso contrário, se deterão se encontrarem alguma partícula parada na sua frente que bloqueie seu movimento. Neste caso, as velocidades voltarão para 0 e as partículas ficarão bloqueadas até que a partícula ou as partículas que bloqueiam seu caminho tenham partido. A partir deste instante, a partícula não bloqueada espera mais um tempo aleatório com distribuição exponencial para mover-se. Em todo instante de tempo teremos partículas que se movem a velocidade -1 ou bloqueadas. Apresentaremos as condições para que cada partícula entre na fase de fluxo livre, isto é, cada partícula entra eventualmente no estado livre e portanto, move-se à velocidade -1 para sempre.

A partir do processo pontual marcado, definiremos as trajetórias das partículas de maneira funcional, isto é, as trajetórias serão uma função do processo pontual. Da trajetória de uma partícula, calcularemos geometricamente o último intervalo de espera para que esta partícula passe ao estado de fluxo livre. Com as posições iniciais das partículas e os últimos intervalos de espera para se converter em livres, construiremos uma fila com um único servidor que, no estado de equilíbrio, nos dará a distribuição final do sistema de partículas.

Como principal resultado deste capítulo provaremos sob a condição $\lambda<1$ que para cada partícula existe um tempo aleatório (finito quase certamente) a partir do qual a partícula passará ao estado livre para sempre. Além disso, provaremos que no limite quando $t \rightarrow \infty$ a distância entre duas partículas consecutivas corresponde quase certamente ao tempo entre duas saídas consecutivas de uma fila estacionária $M / M / 1 / \infty$ associada ao sistema. 


\subsection{Definição do modelo}

Consideremos um sistema de partículas que se movem da direita para a esquerda no sentido contrário ao sentido positivo do eixo de posições. O tempo está dirigido verticalmente de cima para abaixo, as partícula se moverão à velocidade -1 . Denotemos por $C_{n}$ o índice da $n$-ésima partícula.

A dinâmica do processo pontual é definido como uma função de um processo pontual marcado $\mathbb{M}=\left\{\left(x_{n}, \xi_{n, m}\right) ; n, m \in \mathbb{Z}\right\}$. Onde $\left\{x_{n}, n \in \mathbb{Z}\right\}$ é um processo pontual de Poisson de parâmetro $\lambda$ que define a distribuição das posições iniciais das partículas. As seqüências $\left\{\left\{\xi_{n, m} ; m \leq n\right\}, m, n \in \mathbb{Z}\right\},\left\{x_{n}, n \in \mathbb{Z}\right\}$ são independentes. As marcas são as seqüências $\left\{\left\{\xi_{n, m} ; m \leq n\right\}, m, n \in \mathbb{Z}\right\}$ iid com distribuição exponencial de média $1 \mathrm{e}$ definem tempos de espera da $n$-ésima partícula para mudar sua velocidade de 0 a -1 . A seqüência iid $\left\{\tau_{n}=x_{n+1}-x_{n} ; n \in \mathbb{Z}\right\}$ das distâncias entre as partículas tem distribuição exponencial de média $1 / \lambda$ devido a $\left\{x_{n}, n \in \mathbb{Z}\right\}$ ser um processo de Posisson.

Consideremos $\Pi(t)=\left\{\pi_{n}(t), n \in \mathbb{Z}\right\}$ uma seqüência de trajetórias, que são funções lineares contínuas e com derivada em quase todos seus pontos definidas sobre $[0, \infty)$. Associamos a cada partícula uma trajetória. Inicialmente esta trajetória será construída indutivamente assumindo que temos partículas só no semi-eixo positivo. Depois consideraremos o caso duplamente infinito. Considerando que o índice $n$ numera as partículas, a variável $t$ é o tempo, então, $\pi_{n}(t)$ será a posição da partícula $C_{n}$ no tempo $t$. As partículas são numeradas de tal forma que suas posições iniciais cumprem as seguintes desigualdades

$$
0=x_{0}:=\pi_{0}(0)<x_{1}=: \pi_{1}(0)<\ldots<x_{n}=: \pi_{n}(0)<x_{n+1}=: \pi_{n+1}(0) \ldots \ldots
$$

O tempo de espera inicial da partícula $C_{n}$ é definido por $\xi_{n, n}, n \in \mathbb{Z}$ mediante

$$
\dot{\pi}_{n}(t)=0 \text { se } 0<t<\xi_{n, n} .
$$

\section{Definições}

1. Definimos o atraso inicial da partícula $C_{n}$ como o tempo que a partícula espera para mover-se pela primeira vez. Existe $t_{n}^{0}$ tal que

$$
\dot{\pi}_{n}(t)=0 \text { para } 0 \leq t<t_{n}^{0}
$$

O tempo $t_{n}^{0}$ é denominado o atraso inicial da partícula $C_{n}$. Vemos da definição do processo que o atraso inicial de $C_{n}$ é dado por $\xi_{n, n}$ e será denotado por $D_{n, n}$.

2. Dizemos que a partícula $C_{n}$ será bloqueada se existe um $m \leq n-1$ tal que

$$
\pi_{n}(t)=x_{m}\left(:=\pi_{m}(0)\right) \text { e } \dot{\pi}_{n}(t)=0
$$

3. Para $m \leq n-1$ definimos o $m$-ésimo atraso da partícula $C_{n}$ como o tempo que a partícula gasta estando bloqueada pela partícula $C_{n-1}$ na posição $x_{m}\left(:=\pi_{m}(0)\right)$ mais o tempo que a partícula gasta esperando para mudar sua velocidade de 0 a -1 quando não está mais bloqueada. Seja 


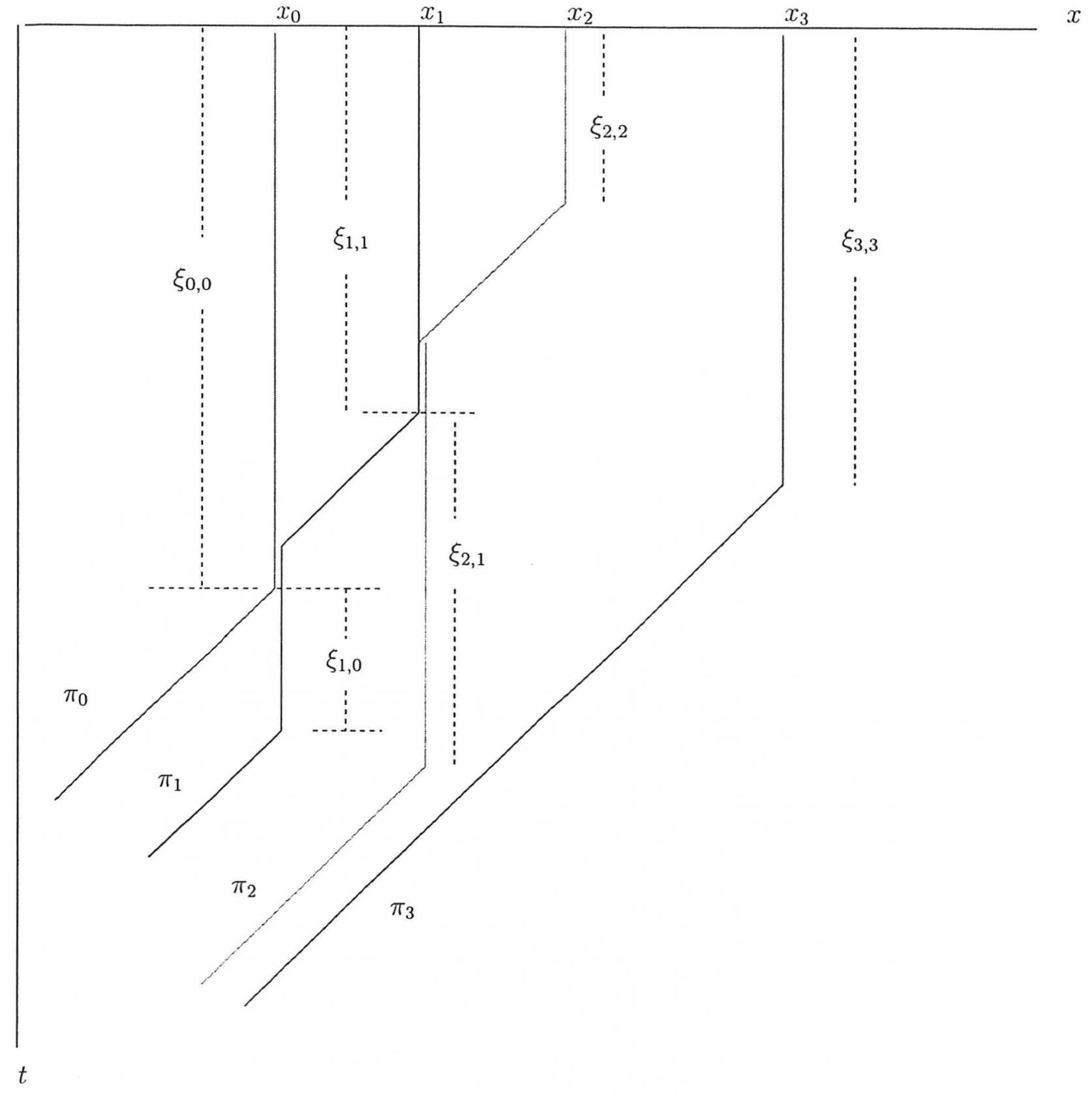

Figura 5.1: Dinâmica do modelo pontual 


$$
\begin{aligned}
& t_{n, m}^{-}=\inf \left\{t: \pi_{n}(t)=\pi_{m}(0) \text { e } \dot{\pi}_{n}(t)=0\right\} \\
& t_{n, m}^{+}=\sup \left\{t: \pi_{n}(t)=\pi_{m}(0) \text { e } \dot{\pi}_{n}(t)=0\right\}
\end{aligned}
$$

O valor $t_{n, m}^{+}-t_{n, m}^{-}$é chamado de $m$-ésimo atraso da partícula $C_{n}$ e será denotado por $D_{n, m}$. Portanto, temos $D_{n, m}:=t_{n, m}^{+}-t_{n, m}^{-}$

4. Dizemos que a partícula $C_{n}$ é livre desde o início se nunca foi bloqueada e move-se à velocidade -1 para sempre. Então, existe $t^{0}(n)$ (atraso inicial de $C_{n}$ ) tal que

$$
\dot{\pi}_{n}(t)=-1 \text { pata todo } t>t^{0}(n):=\xi_{n, n}
$$

5. Dizemos que a partícula $C_{n}$ é livre para sempre se existe um tempo aleatório $t(n)$ que depende de $n$ tal que

$$
\dot{\pi}_{n}(t)=-1 \text { para todo } t>t(n)
$$

Diremos que a partícula não é livre se este tempo é infinito.

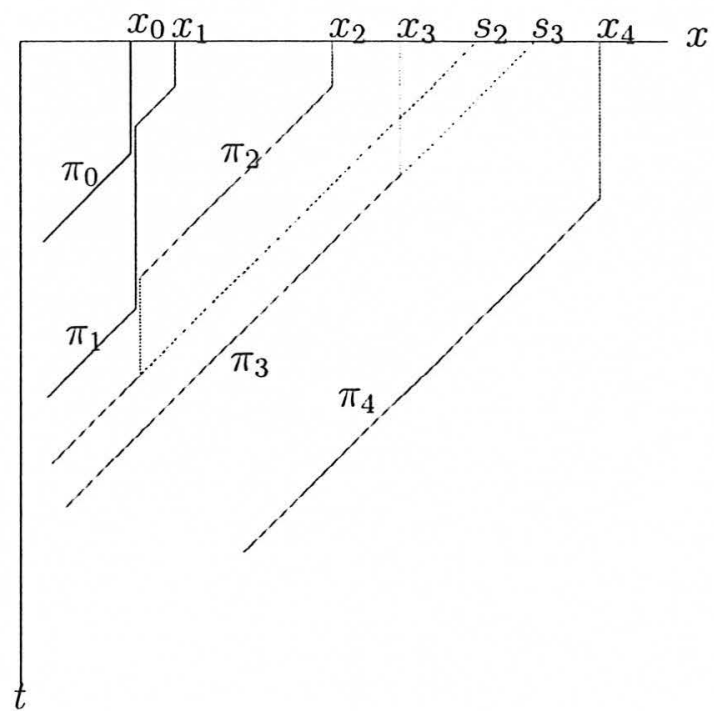

Figura 5.2: Ciclo do Modelo Pontual 


\subsubsection{Construção do Processo em $\mathbb{R}$}

Teorema 0. Se $\lambda<1$ existe uma versão estacionária espacialmente do processo $\left\{\left\{\Pi_{n}(t), t \in \mathbb{R}\right\}, n \in \mathbb{Z}\right\}$.

Prova. A partir do ciclo, como o tamanho do ciclo tem esperança finita (será provado na proposição 1), podemos construir o processo em $\mathbb{R}$, ver Thorisson capítulo 8 [29].

\subsection{Resultados}

Teorema 1. Dado o processo pontual marcado $\mathbb{M}$. Se $\lambda<1$.

a. Então, $\mathbb{P}\left(M_{n}(\mathbb{M})<\infty\right)=1$ e o tempo de espera para que a partícula passe ao estado livre é dado por $T_{M_{n}}=D_{n}+\left(x_{n}-x_{M_{n}}\right)$. Onde $D_{n}=\sum_{m} D_{n, m}$ é a soma do todos os atrasos da partícula $C_{n}$ e $M_{n}(\mathbb{M})$ é definido por

$$
M_{n}(\mathbb{M})=\min \left\{m \leq n: D_{n, m}=t_{n, m}^{+}-t_{n, m}^{-}>0\right\}
$$

b. Além disso,

$$
\lim _{t \rightarrow \infty}\left(\pi_{n}(t)-\pi_{n-1}(t)\right)=\tilde{s}_{n}-\tilde{s}_{n-1} \text { quase certamente }
$$

Onde $\tilde{s}_{n}$ é o tempo de saída do $n$-ésimo cliente de uma fila estacionária $M / M / 1 / \infty$ associada ao sistema.

Teorema 2. Dado o processo pontual marcado $\mathbb{M}$. Se $\lambda<1$.

a. Existe um $T=T(\mathbb{M})<\infty$ aleatório, tal que, se $t>T$, todas as partículas que passam pela origem depois de $T$ são livres.

b. O processo pontual no eixo temporal $X=0$ é Poisson a partir desse $T(\mathbb{M})$

A prova dos teoremas está baseado na representação do modelo de trânsito como uma fila com um único servidor e será realizada no final do capítulo.

\subsection{Construção Gráfica do Modelo Pontual}

\subsubsection{Construção das trajetórias $\Pi($.$) condicionada a C_{0}$ livre desde o início.}

Consideremos o modelo pontual $\mathbb{M}$ definido na seção anterior. As partículas são distribuídas no semi-eixo positivo segundo o processo de Poisson $\left\{x_{n}, n \geq 0\right\}$ de parâmetro $\lambda<1$. As marcas são o tempo de espera para que as partículas mudem sua velocidade 
de 0 a -1 e tem distribuição exponencial de média 1 . Consideremos o movimento das partículas no plano real $\mathbb{R} \times \mathbb{R}_{+}$definido por $\left\{(x, t) ; x \in \mathbb{R}, t \in \mathbb{R}_{+}\right\}$

- Começar com a partícula $C_{0}$ livre desde o início. $C_{0}$ espera $\xi_{0,0}=D_{0,0}$ para mover-se e sua equação de movimento será

$$
\pi_{0}(t)= \begin{cases}0 & \text { se } 0<t \leq \xi_{0,0} \\ -t+\xi_{0,0} & \text { se } t>\xi_{0,0}\end{cases}
$$

$C_{0}$ não é atrasado por nenhuma partícula. Definimos a região $R_{0}^{0}$ como o triângulo formado entre os pontos $(0,0),\left(\xi_{0,0}, 0\right)$ e $\left(0, \xi_{0,0}\right)$

- Considerar um novo ponto $x_{1}=x_{0}+\tau_{0}$, correspondente à partícula $C_{1}$

1. Se $x_{1}>\xi_{0,0}$ definimos $x_{1}:=x_{0}$ e começamos de novo.

2. Se $x_{1}<\xi_{0,0}$ esperamos o tempo aleatório $\xi_{1,1}$

- Se $\left(x_{1}, \xi_{1,1}\right) \in R_{0}^{0}$, então

$$
\pi_{1}(t)= \begin{cases}x_{1} & \text { se } 0<t \leq \xi_{1,1} \\ -t+x_{1}+\xi_{1,1} & \text { se } \xi_{1,1}<t \leq \xi_{1,1}+x_{1} \\ 0 & \text { se } \xi_{1,1}+x_{1}<t \leq \xi_{0,0}\end{cases}
$$

Temos que $C_{1}$ está bloqueado no ponto $\pi_{0}(0)=0$.

Esperar mais um tempo aleatório $\xi_{1,0}$, então, $C_{1}$ estará livre e a sua trajetória completa será.

$$
\pi_{1}(t)= \begin{cases}x_{1} & \text { se } 0<t \leq \xi_{1,1} \\ -t+x_{1}+\xi_{1,1} & \text { se } \xi_{1,1}<t \leq \xi_{1,1}+x_{1} \\ 0 & \text { se } \xi_{1,1}+x_{1}<t \leq \xi_{1,1}+\xi_{1,0}+x_{1} \\ -t+x_{1}+D_{1,1}+D_{1,0} & \text { se } t>x_{1}+D_{1,1}+D_{1,0}\end{cases}
$$

Onde $D_{1,0}$ é o primeiro atraso de $C_{1}$ no ponto 0 e $D_{1,1}:=\xi_{1,1}$. Geometricamente encontramos que $D_{1,0}=\xi_{0,0}+\xi_{1,0}-\xi_{1,1}-x_{1}$. Definimos $D_{1}=D_{1,1}+D_{1,0}$ e $s_{1}:=x_{1}+D_{1}$. $s_{1}$ é a interseção da prolongação da trajetória $\pi_{1}(t)$ com a reta $t=0$.

Esta trajetória de $C_{1}$ gera cluas regiões que serão utilizadas para a construção da trajetória de $C_{2}$.

- Se $\left(x_{1}, \xi_{1,1}\right) \notin R_{0}^{0}$, então, a trajetória de $C_{1}$ será

$$
\pi_{1}(t)= \begin{cases}x_{1} & \text { se } 0<t \leq \xi_{1.1} \\ -t+x_{1}+\xi_{1,1} & \text { se } t>\xi_{1,1}\end{cases}
$$




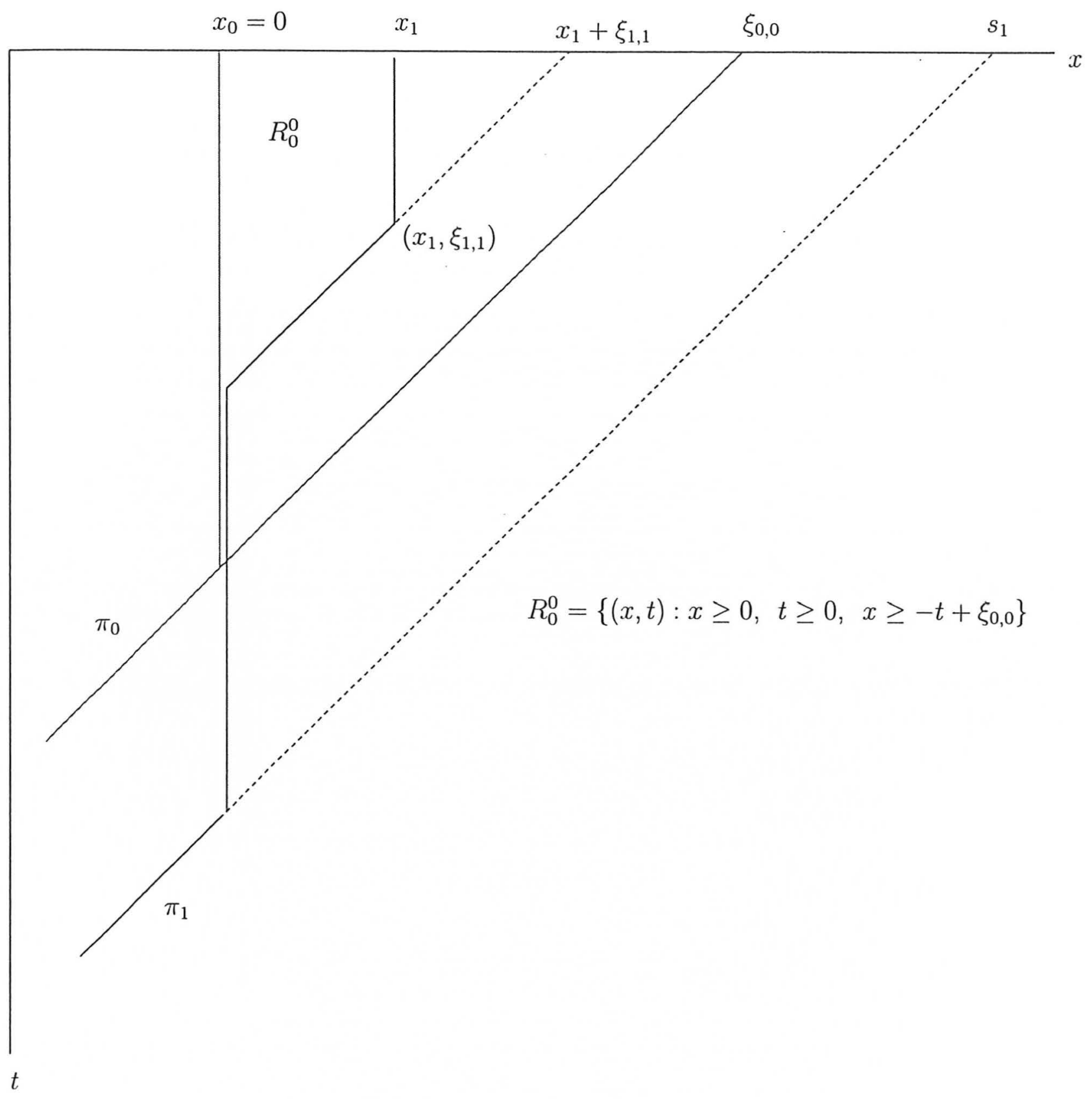

Figura 5.3: Representação Gráfica do Modelo Pontual para $C_{0}$ e $C_{1}$ 
Vemos que neste caso $C_{1}$ é livre desde o início e gera só uma região que será usada para construir a trajetória da partícula $C_{2}$.

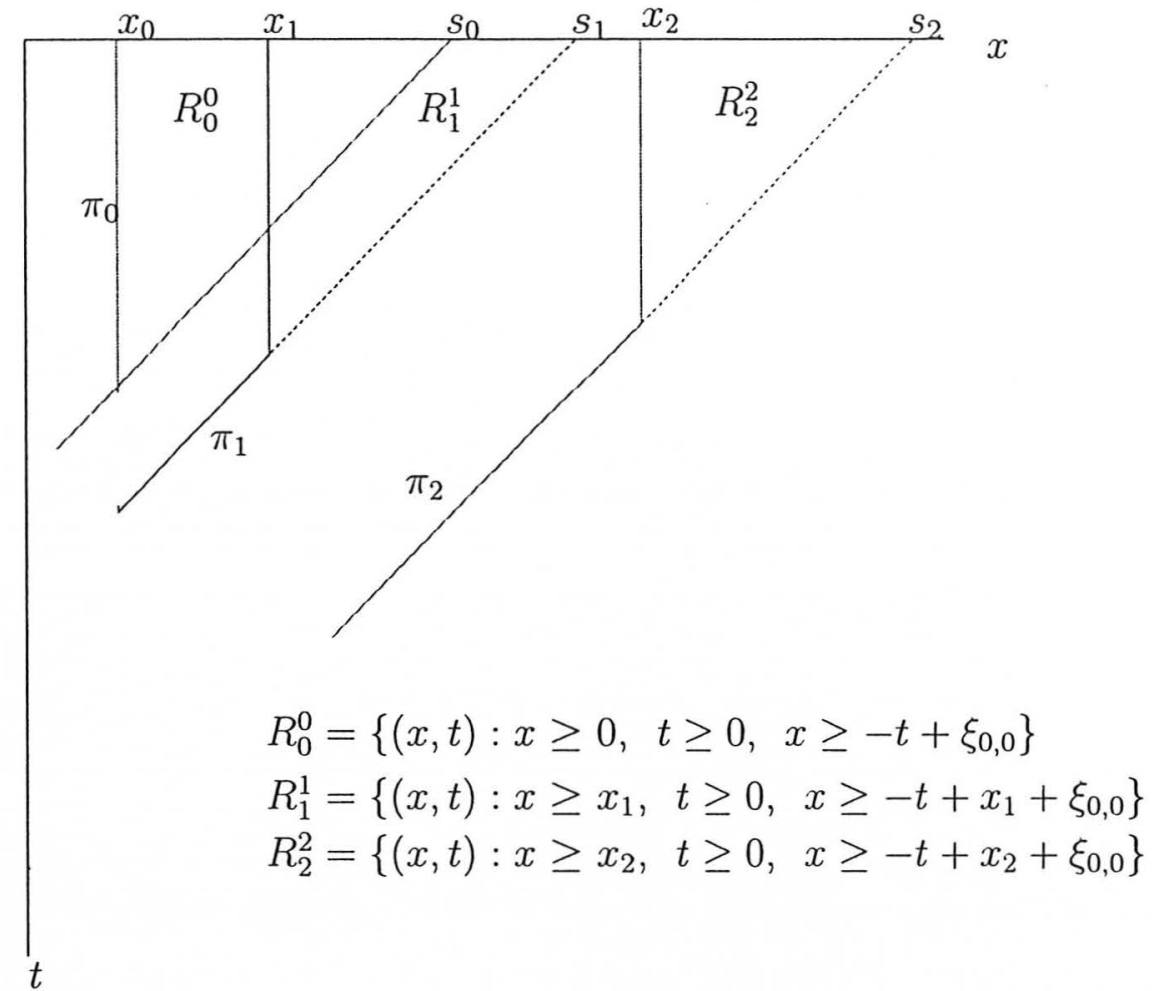

Figura 5.4: Trajetórias de $C_{1}$ livre desde o início e $C_{2}$ livre.

- Introduzir no processo a partícula $C_{n+1}$, definindo $x_{n+1}=x_{n}+\tau_{n}$, para $n>1$. Definimos

$$
\Gamma_{n}=\left\{m_{1}, m_{2}, \ldots, m_{k}, m_{k+1}\right\}: 0 \leq m_{1}<m_{2}<\ldots<m_{k}<m_{k+1}:=n
$$

para todo $k \in \mathbb{N}$, como o conjunto de pontos de atraso da partícula $C_{n}$.

Definimos o atraso total da partícula $C_{n}$ por

$$
D_{n}:=\sum_{k \in \Gamma_{n}} D_{n, k}
$$

Definimos também

$$
s_{n}:=x_{n}+D_{n}
$$

como saída da partícula $C_{n}$. O ponto $s_{n}$ é a interseç̧ão da prolongação da trajetória $\pi_{n}(t)$ definida sobre $t>T_{n, m_{1}}$ com a reta $t=0$. A palavra saída é usada para 
representar que para tempos maiores que $T_{n, m_{1}}$, a partícula deixa de ser bloqueada e passa a ser livre. Aqui, $T_{n, m_{1}}$ é o instante de tempo que a partícula $C_{n}$ deixa o ponto de bloqueio $m_{1}$.

1. Se $x_{n+1}>s_{n}$, então, definimos $x_{n+1}:=x_{0}$ e começamos de novo.

2. Se $x_{n+1}<s_{n}$, então, esperamos um tempo aleatório $\xi_{n+1, n+1}$ e definimos a trajetória da partícula $C_{n+1}$ usando as regiões definidas pela trajetória de partícula $C_{n}$. Isto será calculado na próxima subseção.

- O processo termina sempre que introduzimos uma nova partícula e sua coordenada é maior que a saída da partícula anterior.

\subsubsection{Cálculo da trajetória da partícula $C_{n+1}$ a partir da tra- jetória da partícula $C_{n}$}

Definido $\Gamma_{n}=\left\{m_{1}, m_{2}, \ldots, m_{k}, m_{k+1}\right\}, k \in \mathbb{N}: 0 \leq m_{1}<m_{2}<\ldots<m_{k}<m_{k+1}:=n$, como o conjunto de pontos de atraso da partícula $C_{n}$. Podemos construir a equação da trajetória da partícula $C_{n}$ que é dada por

$$
\pi_{n}(t)= \begin{cases}x_{n} & \text { se } 0<t \leq \xi_{n, n} \\ -t+x_{n}+\xi_{n, n} & \text { se } \xi_{n, n}<t \leq \xi_{n, n}+\left(x_{n}-x_{m_{k}}\right) \\ 0 & \text { se } \xi_{n, n}+\left(x_{n}-x_{m_{k}}\right)<t \leq T_{n, m_{k}} \\ -t+x_{m_{k}}+T_{n, m_{k}} & \text { se } T_{n, m_{k}}<t \leq T_{n, m_{k}}+\left(x_{m_{k}}-x_{m_{k-1}}\right) \\ \vdots & \\ -t+x_{m_{3}}+T_{n, m_{3}} & \text { se } T_{n, m_{3}}<t \leq T_{n, m_{3}}+\left(x_{m_{4}}-x_{m_{3}}\right) \\ 0 & \text { se } T_{n, m_{3}}+\left(x_{m_{4}}-x_{m_{3}}\right)<t \leq T_{n, m_{2}} \\ -t+x_{m_{2}}+T_{n, m_{2}} & \text { se } T_{n, m_{2}}<t \leq T_{n, m_{2}}+\left(x_{m_{2}}-x_{m_{1}}\right) \\ 0 & \text { se } T_{n, m_{2}}+\left(x_{m_{2}}-x_{m_{1}}\right)<t \leq T_{n, m_{1}} \\ -t+x_{m_{1}}+T_{n, m_{1}} & \text { se } t>T_{n, m_{1}}\end{cases}
$$

Definimos por $T_{n, m_{j}} ; j \in\{1,2, \ldots, k\}$ o instante de tempo no qual a partícula $C_{n}$ deixa o ponto $x_{m_{j}}$ depois de estar bloqueada neste ponto. Existe uma relação entre estes tempos de saída dos pontos de bloqueio e os atrasos da partícula $C_{n}$.

$$
T_{n, m_{j}}=\sum_{i=j}^{n} D_{n, m_{i}}+\left(x_{n}-x_{j}\right)
$$

Por definição do processo temos que $\xi_{n, n}=D_{n, n}=T_{n, n}$. O tempo de espera inicial, o atraso inicial e o tempo de saída da partícula $C_{n}$ do ponto $x_{n}$ são todos iguais. Existe também uma relação geométrica entre os tempos de saída das partículas $C_{n}$ e $C_{n-1}$. 


$$
T_{n, m_{j}}=T_{n-1, m_{j}}+\xi_{n, m_{j}}
$$

Da definição da trajetória da partícula $C_{n}$ (equação 5.13) podemos ver facilmente que se prolongamos as retas de saída dos pontos de bloqueio até interceptar a reta $t=0$ se formam $k+1$ regiões. Uma região triangular correspondente ao atraso inicial e $k$ regiões poligonais correspondentes aos $k$ atrasos restantes. Denotaremos estas regiões como $R_{m_{j}}^{n}, j \in\{1,2, \ldots, k, n\}$.

Definimos a região triangular como

$$
R_{n}^{n}:=\left\{(x, t): x \geq x_{n} \text { e } t \geq 0 \text { e } x \geq-t+s_{n}\right\} ; s_{n}=x_{n}+D_{n, n}
$$

$\mathrm{E}$ as outras regiões

$$
R_{m_{j}}^{n}=\left\{(x, t): x \geq x_{m_{j}} \text { e } t \geq 0 \text { e } x \geq-t+x_{n}+\sum_{i=j}^{n} D_{n, m_{i}} \text { e } x \leq-t+x_{n}+\sum_{i=j+1}^{n} D_{n, m_{i}}\right\}
$$

Onde $j \in\{1,2, \ldots, k\}$. Outra forma de definir as regiões é definindo os vértices do trapézio formado. Então, a região $R_{m_{j}}^{n}$ é definido pelos pontos internos aos pontos $\left(s_{j}, 0\right)$, $\left(s_{j+1}, 0\right),\left(m_{j}, T_{n, m_{j+1}}+\left(x_{m_{j+1}}-x_{m_{j}}\right)\right)$ e o ponto $\left(m_{j}, T_{n, m_{j}}\right)$.

Segundo o algoritmo estabelecido, introduzimos a partícula $C_{n+1}$ somando uma variável exponencial à posição da partícula $C_{n}$, isto é, $x_{n+1}=x_{n}+\tau_{n}$.

1. Se $x_{n+1}>s_{n}$ o processo termina e começamos de novo definindo $x_{n+1}:=x_{0}$.

2. Se $x_{n+1}<s_{n}$, então, esperamos um tempo aleatório igual a $\xi_{n+1, n+1}$.

- Se $\left(x_{n+1}, \xi_{n+1, n+1}\right) \notin \cup_{j} R_{m_{j}}^{n}$, então, a partícula será livre desde o início e a sua trajetória $\pi_{n+1}(t)$ será

$$
\pi_{n+1}(t)= \begin{cases}x_{n+1} & \text { se } 0<t \leq \xi_{n+1, n+1} \\ -t+x_{n+1}+\xi_{n+1, n+1} & \text { se } t>\xi_{n+1, n+1}\end{cases}
$$

- Se $\left(x_{n+1}, \xi_{n+1, n+1}\right) \in \cup_{j} R_{m_{j}}^{n}$ verificamos a que região pertence o ponto $\left(x_{n+1}, \xi_{n+1, n+1}\right)$. Supondo que pertence à região $R_{m_{2}}^{n}$, então, a trajetória para $\pi_{n+1}(t)$ será

$$
\pi_{n+1}(t)= \begin{cases}x_{n+1} & \text { se } 0<t \leq \xi_{n+1, n+1} \\ -t+x_{n+1}+\xi_{n+1, n+1} & \text { se } \xi_{n+1, n+1}<t \leq \xi_{n+1, n+1}+\left(x_{n+1}-x_{m_{2}}\right) \\ 0 & \text { se } \xi_{n+1, n+1}+\left(x_{n+1}-x_{m_{2}}\right)<t \leq T_{n, m_{2}}\end{cases}
$$

Neste ponto $x_{m_{2}}$ esperamos mais um tempo aleatório igual a $\xi_{n, m_{2}}$. 


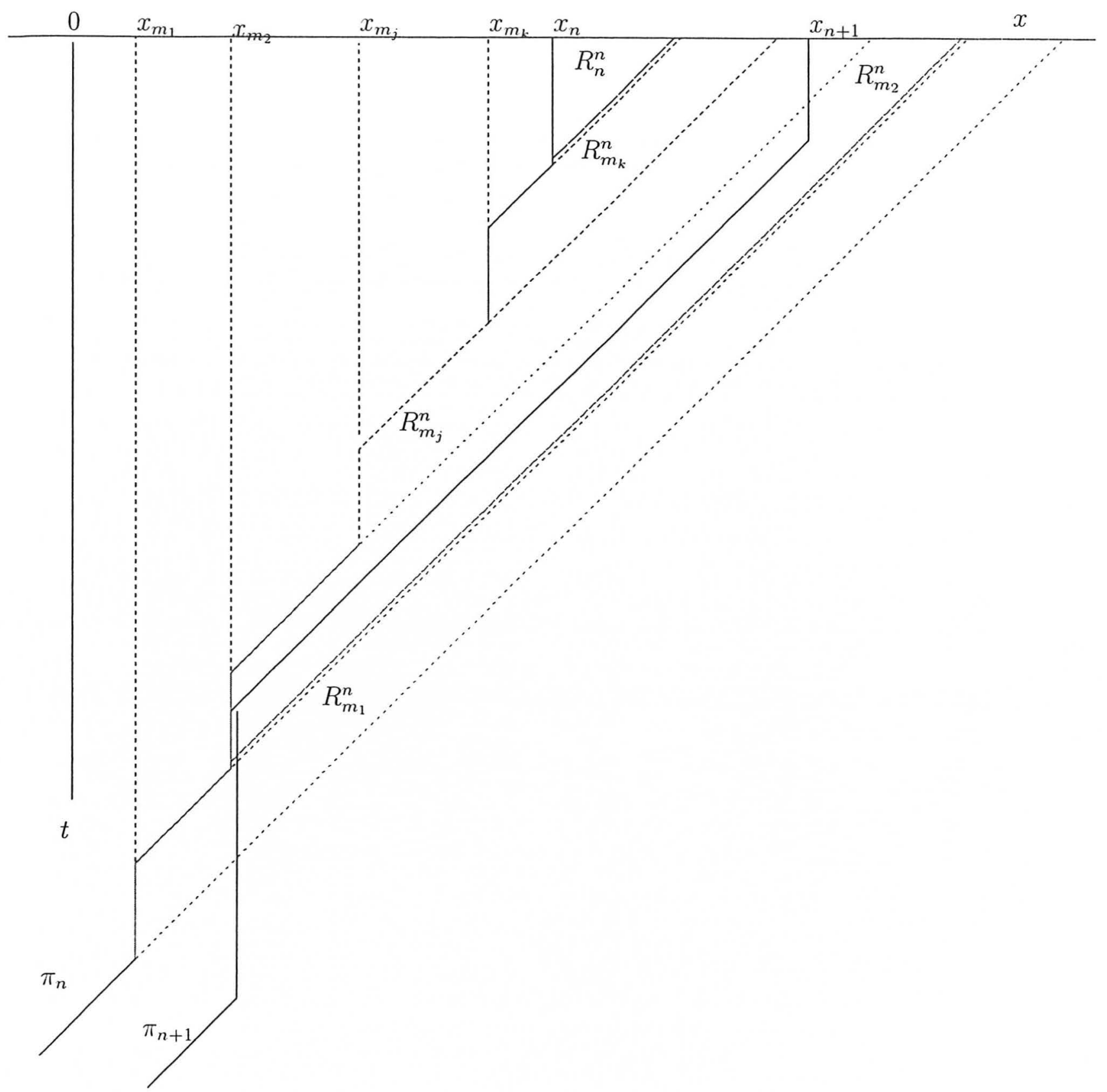

Figura 5.5: Trajetória da partícula $C_{n+1}$ a partir de $C_{n}$. 
Se $\left(m_{j}, T_{n, m_{2}}+\xi_{n, m_{2}}\right) \notin R_{m_{1}}^{n}$, então, a partícula fica livre e não é bloqueada no ponto $x_{m_{1}}$. Neste caso, a equação da trajetória completa será

$$
\pi_{n+1}(t)= \begin{cases}x_{n+1} & \text { se } 0<t \leq \xi_{n+1, n+1} \\ -t+x_{n+1}+\xi_{n+1, n+1} & \text { se } \xi_{n+1, n+1}<t \leq \xi_{n+1, n+1}+\left(x_{n+1}-x_{m_{2}}\right) \\ 0 & \text { se } \xi_{n+1, n+1}+\left(x_{n+1}-x_{m_{2}}\right)<t \leq T_{n, m_{2}}+\xi_{n, m_{2}} \\ -t+x_{n+1}+D_{n+1} & \text { se } t>T_{n, m_{2}}\end{cases}
$$

Caso contrário, $\left(m_{j}, T_{n, m_{2}}+\xi_{n, m_{2}}\right) \in R_{m_{1}}^{n}$ a partícula será bloqueada por última vez no ponto $x_{m_{1}}$. Neste último ponto de bloqueio espera mais um tempo aleatório igual a $\xi_{n, m_{1}}$ e finalmente fica livre e a equação completa da trajetória será

$$
\pi_{n+1}(t)= \begin{cases}x_{n+1} & \text { se } 0<t \leq \xi_{n+1, n+1} \\ -t+x_{n+1}+\xi_{n+1, n+1} & \text { se } \xi_{n+1, n+1}<t \leq \xi_{n+1, n+1}+\left(x_{n+1}-x_{m_{2}}\right) \\ 0 & \text { se } \xi_{n+1, n+1}+\left(x_{n+1}-x_{m_{2}}\right)<t \leq T_{n, m_{2}}+\xi_{n, m_{2}} \\ -t+x_{m_{2}}+T_{n, m_{2}}+\xi_{n, m_{2}} & \text { se } T_{n, m_{2}}+\xi_{n, m_{2}}<t \leq T_{n+1, m_{2}}+\left(x_{m_{2}}-x_{m_{1}}\right) \\ 0 & \text { se } T_{n+1, m_{2}}+\left(x_{m_{2}}-x_{m_{1}}\right)<t \leq T_{n, m_{1}} \\ -t+x_{n+1}+D_{n+1} & \text { se } t>T_{n, m_{1}}\end{cases}
$$

\subsubsection{A trajetórias $\Pi($.$) como função do processo \mathbb{M}$ condicionada a $C_{0}$ livre desde o início}

Consideremos o modelo pontual $\mathbb{M}$ definido na seção anterior. As partículas são distribuídas no semi-eixo positivo segundo o processo de Poisson $\left\{x_{n}, n \geq 0\right\}$ de parâmetro $\lambda<1$. As marcas são o tempo de espera para que as partículas mudem sua velocidade de 0 a -1 e tem distribuição exponencial de média 1 . Consideremos o movimento das partículas no plano real $\mathbb{R} \times \mathbb{R}_{+}$definido por $\left\{(x, t) ; x \in \mathbb{R}, t \in \mathbb{R}_{+}\right\}$

Usando as equações para $\left\{\pi_{n}, n \in \mathbb{Z}\right\}$ obtidas na subseção anterior, construiremos as trajetórias das partículas como uma função do processo pontual $\mathbb{M}$. Encontraremos para cada partícula o tempo de espera total e o último intervalo de espera para ser livre para sempre.

Definimos por $\sigma_{n}$ o último intervalo de tempo que a partícula $C_{n}$ espera para ser livre para sempre. A seqüência $\left\{\sigma_{n}, n \in \mathbb{Z}\right\}$ será a seqüência dos últimos intervalos de tempo de espera das partículas para serem livres para sempre.

- Para $C_{0}$ temos 


$$
\pi_{0}(t)=\Phi\left(\left(x_{0}\right),\left(\xi_{0,0}\right)\right)
$$

Onde $\Phi$ é uma função definida do conjunto $\left\{\cup_{k \geq 1}^{n}\left(\mathbb{R}_{+} \times \mathbb{R}_{+}\right)^{k}\right\} \times\left(\mathbb{R}_{+}\right)^{n+1}, n \in \mathbb{N}$, para o conjunto $\left\{\Pi_{n}, n \in \mathbb{Z}\right\}$

Obtemos da trajetória $\sigma_{0}=\xi_{0,0}$ e $D_{0,0}=\xi_{0,0}$ é o atraso inicial.

- Para $C_{1}$ temos

$$
\pi_{1}(t)=\Phi\left(\left\{\left(x_{0}, x_{1}\right),\left(D_{0,0}, D_{1,0}\right) ; \xi_{1,0}\right\}\right)
$$

ou escrito de outra maneira

$$
\pi_{1}(t)=\Phi\left(\left\{\left(x_{0}\right),\left(D_{0,0}\right)\right\} ;\left(x_{1}, \xi_{1,1}, \xi_{1,0}\right)\right)
$$

Observemos que $\pi_{1}(t)$ é uma função do ponto $\left(x_{0}, D_{0,0}\right)$, onde $x_{0}$ será ponto de possível atraso e $D_{0,0}$ se usará para calcular o atraso de $C_{1}$ em $x_{0}$ que denotamos por $D_{1,0}$. Também é função de $\left(x_{1}, \xi_{1,1}, \xi_{1,0}\right)$, sendo $x_{1}$ sua coordenada e $\xi_{1,1}$ seu atraso inicial e $\xi_{1,0}$ é o intervalo de espera para sair do bloqueio em $x_{0}$.

Obtemos da trajetória

$$
\sigma_{1}= \begin{cases}\xi_{1,0} & \text { se } \xi_{1,1}<\xi_{0,0}-\tau_{0} \\ \xi_{1,1}-\xi_{0,0}+\tau_{0} & \text { se } \xi_{1,1}>\xi_{0,0}-\tau_{0}\end{cases}
$$

Ademais, geometricamente obtemos

$$
\begin{aligned}
D_{1,0} & =\xi_{0,0}-\tau_{0}-\xi_{1,1}+\xi_{1,0} \\
D_{1} & =\xi_{1,0}+\xi_{0,0}-\tau_{0} \\
T_{1,0} & =\xi_{1,0}+\xi_{0,0}
\end{aligned}
$$

$T_{1,0}$ é o tempo aleatório que $C_{1}$ espera para ficar livre para sempre.

- Para $C_{2}$ temos

$$
\pi_{2}(t)=\Phi\left(\left\{\left(x_{0}, x_{1}\right),\left(D_{1,1}, D_{1,0}\right)\right\} ;\left(x_{2}, \xi_{2,2}, \xi_{2,1}, \xi_{2,0}\right)\right)
$$

Vemos que a trajetória de $C_{2}$ é função dos pontos de atraso de $C_{1}$, da sua própria coordenada e seus intervalos de espera para sair dos bloqueios que a partícula $C_{1}$ lhe impõem. 
Obtemos $\sigma_{2}$ como uma função das trajetórias $\pi_{0}$ e $\pi_{1}$. Definimos por $\mathcal{H}$ o espaço das trajetórias $\Pi($.$) , então, a função G: \mathcal{H} \times \mathcal{H} \rightarrow \Omega$ onde $\Omega$ é o domínio da seqüência das variáveis aleatórias $\left\{\sigma_{n}, n \in \mathbb{Z}\right\}$. Logo,

$$
\sigma_{2}=G\left(\pi_{2}, \pi_{1}\right)
$$

Os pontos de atraso para $C_{2}$ são dados por $\Gamma_{2}=\left\{m_{1}, m_{2}, m_{3}\right\}$ onde $0 \leq, m_{1}<$ $m_{2}<m_{3}:=2$. Se $m_{1}=1$, então $m_{2}$ não existe.

- Para $C_{n+1}, n>1$.

Para calcular a forma funcional da trajetória de $C_{n+1}$ temos que usar os pontos de atraso da partícula $C_{n}$. Seja $\Gamma_{n}=\left\{m_{1}, m_{2}, \ldots, m_{k}, m_{k+1}\right\}, k \in \mathbb{N}$, tal que, $0 \leq m_{1}<$ $m_{2}<\ldots, m_{k}<m_{k+1}:=n$ o conjunto de pontos de atraso da partícula $C_{n}$. Teremos que

$$
\begin{gathered}
\pi_{n+1}(t)=\Phi\left(\left\{\left(x_{m_{1}}, x_{m_{2}}, \ldots, x_{n}\right),\left(D_{n, m_{1}}, D_{n, m_{2}}, \ldots, D_{n, m_{k}}, D_{n, n}\right)\right\}\right. \\
\left.\quad ;\left(x_{n+1}, \xi_{n+1, m_{1}}, \xi_{n+1, m_{2}}, \ldots, \xi_{n+1, n}, \xi_{n+1, n+1}\right)\right)
\end{gathered}
$$

Obtemos da trajetória que

$$
\sigma_{n+1}=G\left(\pi_{n+1}, \pi_{n}\right)
$$

Supondo que $C_{n+1}$ se atrasa por última vez no ponto $m_{j}, j \in\{1,2 \ldots, k, n\}$, então, o tempo aleatório que espera $C_{n+1}$ para ficar livre será

$$
T_{n+1, m_{j}}=D_{n+1}+\left(x_{n+1}-x_{m_{j}}\right)
$$

Onde $D_{n+1}$ é o atraso total de $C_{n+1}$. Se prolongamos a última reta da trajetória de $C_{n+1}$, encontramos na intersecção com a reta $t=0$ o ponto $s_{n+1}$ definido na seção anterior como saída da partícula $C_{n+1}$. Definindo a função $g: \mathcal{H} \rightarrow \mathbb{R}_{+}$, então,

$$
s_{n+1}=g\left(\pi_{n+1}\right) ; \quad s_{n+1}=x_{n+1}+D_{n+1}
$$

Onde $D_{n+1}$ se obtém a partir de $\Gamma_{n}$.

Teorema 3. Dado o processo pontual marcado $\mathbb{M}=\left\{\left(x_{n}, \xi_{n, m}\right) ; n, m \in \mathbb{Z}\right\}$ com seqüências independentes entre si. Onde $\left\{x_{n}, n \in \mathbb{Z}\right\}$ é um processo pontual de Poisson de parâmetro $\lambda<1 ; x_{n}$ são as posições iniciais das partículas. $\left\{\left\{\xi_{n, m}, m \leq n\right\}, m, n \in \mathbb{N}\right\}$ é uma sequiência iid com distribuição exponencial de média 1 e define os tempos de espera para que a $n$-ésima partícula mude sua velocidade de 0 a -1 .

Definindo $\sigma_{n}=G\left(\pi_{n}, \pi_{n-1}\right)$. Então, $\left\{\sigma_{n}, n \in \mathbb{N}\right\}$ é uma seqüência iid com distribuição exponencial de média 1 . 

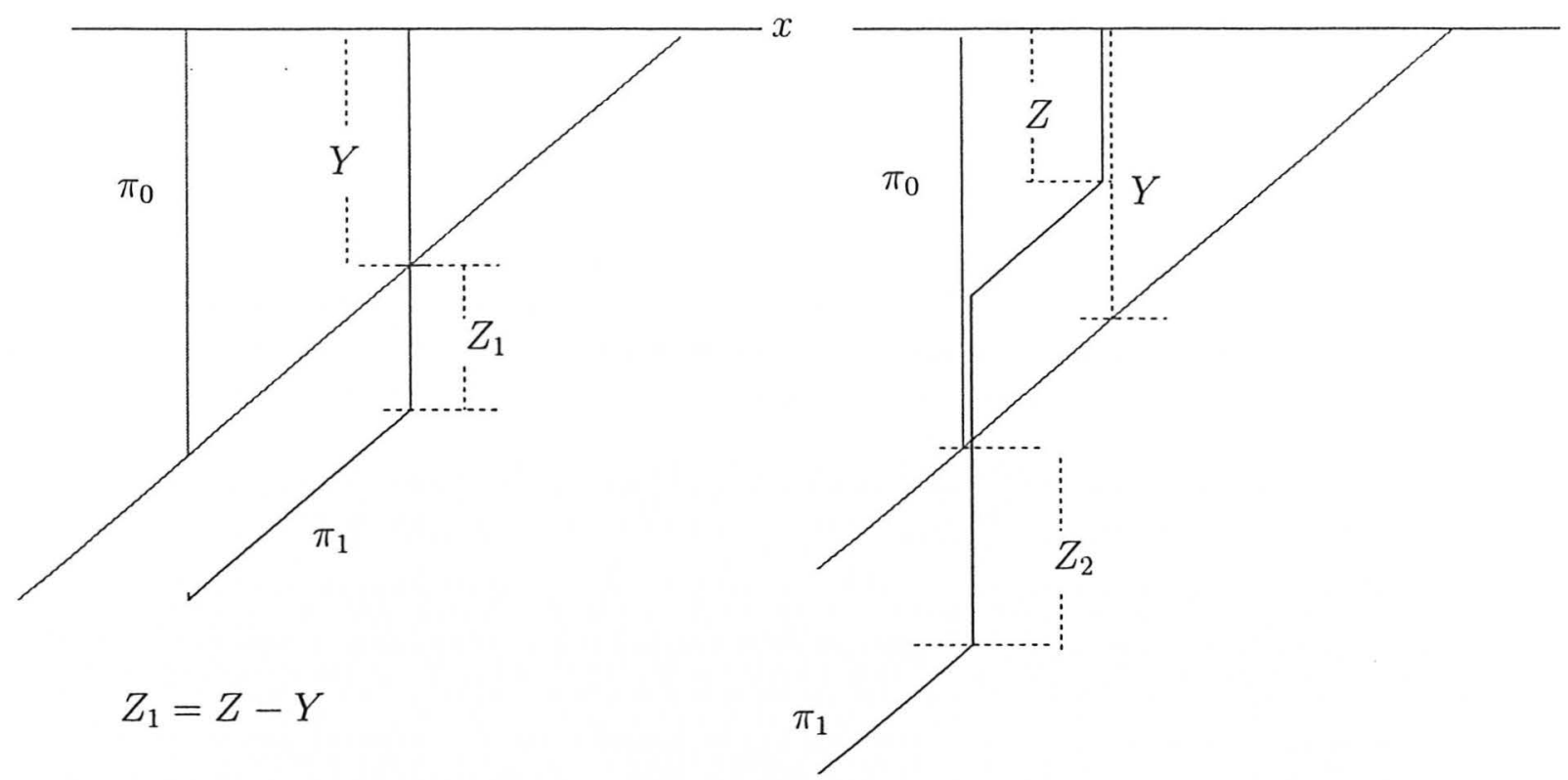

Figura 5.6: Cálculo de $\sigma_{1}$.

\section{Prova.}

A prova será feita usando a representação gráfica do modelo pontual. Dada a trajetória da partícula $C_{0}$ consideremos para a trajetória da partícula $C_{1}$ os dois casos: Primeiro quando $C_{1}$ é livre desde o início e segundo quando $C_{1}$ está bloqueada pela partícula $C_{0}$.

Observemos da figura 5.6 que $\sigma_{1}$ tem a seguinte forma

$$
\sigma_{1}=Z_{1} 1\{Z>Y\}+Z_{2} 1\{Z<Y\}
$$

onde $Z_{1}, Z_{2}$ são exponenciais com média 1 e $Y$ é uma variável aleatória arbitrária. Calculando a distribuição de $\sigma_{1}$ temos

$$
\mathbb{P}\left(\sigma_{1}>x\right)=\mathbb{P}\left(\sigma_{1} \mid Z>Y\right) \mathbb{P}(Z>Y)+\mathbb{P}\left(\sigma_{1} \mid Z<Y\right) \mathbb{P}(Z<Y)
$$

Usando a definição de $\sigma_{1}$ temos

$$
\mathbb{P}\left(\sigma_{1}>x\right)=\mathbb{P}\left(Z_{1}>x\right) \mathbb{P}(Z>Y)+\mathbb{P}\left(Z_{2}>x\right) \mathbb{P}(Z<Y)
$$

Como $Z_{1}$ e $Z_{2}$ são exponenciais temos

$$
\mathbb{P}\left(\sigma_{1}>x\right)=e^{-x}[\mathbb{P}(Z>Y)+\mathbb{P}(Z<Y)]=e^{-x}
$$

Temos provado que $\sigma_{1}$ é exponencial de média 1. 
No caso geral, a prova é idêntica. Por exemplo, para calcular $\sigma_{n}$, temos que definir primeiro a trajetória de $C_{n-1}$, então, dada a trajetória da partícula $C_{n-1}$ calcularemos da trajetória da partícula $C_{n}$ a forma de $\sigma_{n}$. O cálculo da distribuição de $\sigma_{n}$ é idêntica ao feito para $\sigma_{1}$.

\subsection{Fila $M / M / 1 / \infty$ FIFO}

Daremos uma rápida introdução a um modelo de filas. Por modelo de filas entendemos um sistema aberto ao qual clientes de fora entram no sistema, gastam algum tempo dentro deste e logo saem. Durante sua permanência, experimentam congestionamento devido à presença de outros clientes que estão à espera de serviço e então acontece o que chamamos de fila de espera. Uma fila tem cinco características: processo de chegada; processo de serviço; número de servidores; capacidade da fila e forma de atendimento. Kendall criou uma notação que expressa estas características mediante o arranjo $A / B / c / d / X$. A fila $M / M / 1 / \infty$ FIFO é uma fila que tem como processo de entradas um processo pontual de Poisson; o processo de serviços é também um processo pontual de Poisson; tem um único servidor; capacidade infinita (sala de espera infinita) e a tipo de serviço é FIFO (o primeiro cliente em entrar é o primeiro a ser atendido).

\section{Notação}

- $\tilde{C}_{n}$ Denota o $n$-ésimo cliente chegando ao sistema, $n \in \mathbb{Z}$

- $t_{n}$ Tempo de chegada do cliente $\tilde{C}_{n}$

- $\tilde{\tau}_{n}=t_{n+1}-t_{n}$, é o $n$-ésimo tempo entre chegadas

- $\tilde{\sigma}_{n}$ é o tempo de serviço trazido pelo cliente $\tilde{C}_{n}$

- $\tilde{s}_{n}$ tempo de saída do cliente $\tilde{C}_{n}$

Podemos construir esta fila usando um processo pontual marcado $\left\{\left(\tilde{\tau}_{i}, \tilde{\sigma}_{i}\right) ; i \in \mathbb{Z}\right\}$. As seqüências são independentes; $\left\{t_{i} ; i \in \mathbb{Z}\right\}$ é um processo pontual de Poisson de parâmetro $\lambda$. A seqüência $\left\{\tilde{\tau}_{i}:=t_{i+1}-t_{i}, n \in \mathbb{Z}\right\}$ tempo entre chegadas são iid com distribuição exponencial de média $1 / \lambda$. As marcas $\left\{\tilde{\sigma}_{i} ; i \in \mathbb{Z}\right\}$ são seqüências iid com distribuição exponencial de média 1. Assumiremos que o índice que numera os clientes é crescente, quer dizer, que o cliente $\tilde{C}_{n+1}$ entra no sistema depois que o cliente $\tilde{C}_{n}$. Cada cliente $\tilde{C}_{n}$ carrega sua marca $\tilde{\sigma}_{n}$ que será seu tempo de serviço na fila.

Construiremos o processo denominado tempo de espera virtual $\{W(t), t \in \mathbb{R}\}$, também chamado de workload, que é definido como a quantidade de serviço por realizar pelo servidor no tempo $t$ ou pode ser entendido como:o tempo que um cliente virtual esperaria para ser atendido se entrasse na fila no instante $t$. Por construção este processo será de saltos e contínuo à direita com limite à esquerda $W(t-), t \in \mathbb{R}$. O processo $W(t)$ tem saltos nos pontos $t_{n}$, onde $t_{n}$ é o tempo de chegada do cliente $\tilde{C}_{n}$. 

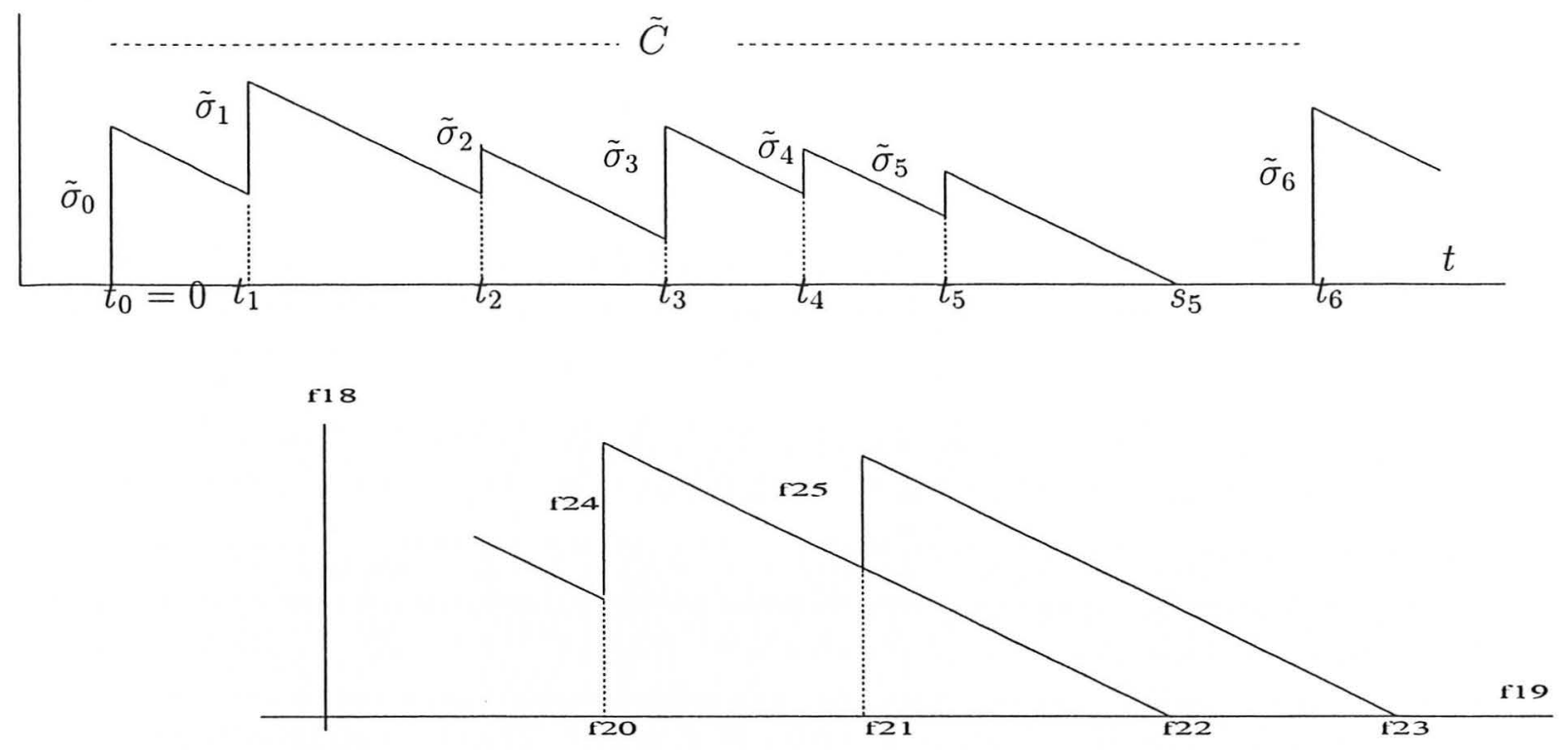

Figura 5.7: Tempo de Espera Virtual $W($.$) e Ciclo da Fila \tilde{C}$

\subsubsection{Construção de um ciclo}

Supondo que inicialmente a fila está vazia e que nenhum cliente chega antes de tempo $t_{0}=0$. Teremos que $W($.$) é 0$ até a chegada de $\tilde{C}_{0}$, então, $W($.$) da um salto de tamanho$ $\tilde{\sigma}_{0}$. O valor de $W($.$) decresce linearmente com inclinação -1$ até a chegada do cliente $\tilde{C}_{1}$ ou alcançar o valor 0 . Se no instante $t$ o workload $W(t)$ alcança 0 , então, este se mantém no valor 0 até a chegada de um novo cliente.

O período ocupado do servidor é o intervalo de tempo durante o qual o servidor está continuamente servindo clientes, quer dizer, que se o cliente $\tilde{C}_{0}$ chega à fila e encontra o servidor desocupado, então, um período começa no instante de tempo $t_{0}=0$, e se $\tilde{s}_{m}$ com $m>0$ é o primeiro momento depois de 0 no qual $W\left(\tilde{s}_{m}\right)=0$. Então, todos os clientes que chegaram durante o intervalo $\left[0, t_{m}\right]$ tem saído. O período ocupado termina no instante $\tilde{s}_{m}$ e sua duração é $\tilde{s}_{m}$. Períodos desocupados são os intervalos de tempo nos quais o servidor está desocupado.

Definimos um ciclo $\tilde{C}$ da fila como o período de tempo ocupado mais o período desocupado adjacente. A fila pode-se representar por ciclos que são formados por períodos ocupados e períodos desocupados adjacentes (ver figura 5.7).

Definimos $W($.$) no intervalo (-\infty, \infty)$. O cliente $C_{0}$ chega à fila no instante $t_{0}=0$ quando a fila está vazia. Sobre o intervalo $\left[0, t_{1}\right)$ o processo é de definido por 


$$
W(t)= \begin{cases}0, & \text { se }-\infty<t<0 \\ \sigma_{0} & \text { se } t=0 \\ {\left[\sigma_{0}-t\right]_{+}} & \text {se } 0<t<t_{1} \\ {\left[\sigma_{0}-\tau_{0}\right]_{+}+\sigma_{1}} & \text { se } t=t_{1}\end{cases}
$$

Onde $[a]_{+}=\max \{0, a\}, a \in \mathbb{R}$. Se construímos $W($.$) sobre \left[0, t_{1}\right]$ podemos, então, definir $W\left(\right.$.) sobre o intervalo $\left[t_{n}, t_{n+1}\right) ; n \geq 1$.

$$
W(t)= \begin{cases}W\left(t_{n}-0\right)+\sigma_{n} & \text { se } t=t_{n} \\ {\left[W\left(t_{n}\right)-\left(t-t_{n}\right)\right]_{+}} & \text {se } t_{n}<t<t_{n+1}\end{cases}
$$

Remarcamos que o valor absoluto do salto nos pontos $t_{i}, i \geq 0$, são definidos como segue:

$$
\sigma_{i}=W\left(t_{i}\right)-W\left(t_{i}-0\right)
$$

Definamos o tempo de espera $w_{n}:=W\left(t_{n}-0\right)$ como o tempo que se passa entre a entrada de cliente $\tilde{C}_{n}$ à fila e o momento em que começa seu serviço. Para estes tempos de espera pode-se obter a relação de recorrência $w_{n+1}=\left[w_{n}+\tilde{\sigma}_{n}-\tilde{\tau}_{n}\right]_{+}$, para $n \geq 0$, com $w_{0}=0$. Esta relação é conhecida como fórmula de Lindley.

Podemos construir o processo das saídas do sistema calculando o instante de saída de cada cliente. Seja $\tilde{s}_{n}$ o instante de saída do cliente $\tilde{C}_{n}$. Dado que o cliente $\tilde{C}_{n}$ entrou no instante $t_{n}$, espero na fila $w_{n}$ e logo demorou o tempo $\tilde{\sigma}_{n}$ no atendimento. Seu tempo de saída será $\tilde{s}_{n}=t_{n}+w_{n}+\tilde{\sigma}_{n}$, para $n \geq 0$, com $w_{0}=0$.

\subsubsection{Teoremas e Relações}

Discutiremos o teorema de estabilidade da fila demonstrado por Loynes que será usado na construção gráfica do modelo pontual.

Clientes chegam ao sistema nos instantes de tempo $\left\{t_{n} ; n \in \mathbb{Z}\right\}$ com tempo de serviço igual a $\left\{\tilde{\sigma}_{n} ; n \in \mathbb{Z}\right\}$. O tempo de espera $w_{n+1}$ do cliente $\tilde{C}_{n+1}$ é expresso pela relação de Lindley.

$$
w_{n+1}= \begin{cases}w_{n}+\tilde{\sigma}_{n}-\tilde{\tau}_{n} & \text { se } w_{n}+\tilde{\sigma}_{n}<\tilde{\tau}_{n} \\ 0 & \text { se } w_{n}+\tilde{\sigma}_{n} \leq \tilde{\tau}_{n}\end{cases}
$$

Para todo tempo $n \in \mathbb{Z}$.

Seja $\mathrm{N}_{0}=\left\{\left(\tilde{\tau}_{\mathbf{n}}, \tilde{\sigma}_{\mathbf{n}}\right) ; \mathbf{n} \in \mathbb{Z}\right\}$, uma seqüência de variáveis iid independentes entre si tal que

$$
\ldots .<t_{-2}<t_{-1}<t_{0}=0<t_{1}<t_{2}<\ldots .
$$

Seja $\mathbf{N}=\left\{\left(\tau_{\mathbf{n}}^{*}, \tilde{\sigma}_{\mathbf{n}}\right) ; \mathbf{n} \in \mathbb{Z}\right\}$, uma seqüência de variáveis iid independentes entre si tal que

$$
\ldots .<r_{-2}<r_{-1} \leq 0<r_{0}<r_{1}<r_{2}<\ldots
$$


Onde $\left\{\tau_{n}^{*}:=r_{n+1}-r_{n}, n \in \mathbb{Z}\right\}$ é uma seqüência iid exponencial de média $1 / \lambda$.

Teorema 4 [Estabilidade da fila]. Considerando $\mathrm{N}_{0}$ uma versão sincronizada (versão de Palm ) do processo $\mathrm{N}$ que é ergódico e estacionário. Se $\mathbb{E} \tilde{\sigma}_{n}-\mathbb{E} \tilde{\tau}_{n}<0$. Então, o processo $\left\{w_{n}, n \in \mathbb{Z}\right\}$ é estável. Isto é, a distribuição de $w_{n}$ quando $n \rightarrow \infty$ converge para uma variável aleatória finita $\phi$, independentemente da condição inicial. Além disso, existe um número infinito de índices negativos $n$ e um infinito número infinito de índices positivos $n$ tais que $w_{n}=0$

Prova Ver o artigo original de Loynes[21] ou uma prova diferente usando a noção de ciclo estacionário feita por Thorisson[29].

Teorema 5 [Processo de saídas, Burke]. Em estado estável(equilíbrio) o processo de saídas da fila $M / M / 1 / \infty$ é um processo de Poisson com parâmetro $\lambda$, e o número de de clientes na fila no instante $t$ é independente do processo de saídas antes do tempo $t$, para cada $t \in \mathbb{R}_{+}$

Prova. Ver Prabhu[24], theorem 8 (página 98).

\subsection{Modelo de Filas Associado ao Modelo Pontual}

Introduzimos uma fila com um único servidor associada ao modelo pontual que denominaremos fila associada ao modelo pontual. Para definir a fila associada introduzimos o processo pontual marcado $\Psi_{0}$ que é uma função das trajetórias $\Pi($.$) , definido por$ $\Psi_{\mathbf{0}}=\left\{\left(\tau_{i}, \sigma_{i}\right) ; i \in \mathbb{Z}\right\}$, com seqüências independentes entre si. Os tempos entre chegadas são dados pela seqüência $\left\{\tau_{i}:=x_{i+1}-x_{i}, i \in \mathbb{Z}\right\}$ que são iid com distribuição exponencial com média $1 / \lambda ;\left\{\sigma_{i}, i \in \mathbb{Z}\right\}$ dada pela equação 5.31 é uma seqüência iid com distribuição exponencial com média 1 (ver teorema 3). A direção positiva do tempo é a usual de esquerda para direita. Assumiremos que o processo pontual de Poisson está ordenado de maneira crescente à direita, quer dizer, que o cliente $\tilde{C}_{n}$ chega no instante $x_{n}$ antes do cliente $\tilde{C}_{n+1}$ que chega no instante $x_{n+1}$. As marcas $\sigma_{n}$ pertencem ao cliente $\tilde{C}_{n}$ e são os tempos de serviço na fila. Na fila associada podemos ver que $\tilde{C}_{n}$ corresponde à partícula $C_{n}$ e $x_{n}$ é a posição inicial desta partícula. Na fila associada não faremos distinção entre $\tilde{C}_{n}$ e $C_{n}$ nem entre o tempo de chegada do cliente $n$-ésimo e a sua posição denotada ambos por $x_{n}$. O eixo dos tempos para a fila associada corresponde ao eixo das posições das partículas. O sentido positivo do tempo na fila associada corresponde ao sentido positivo das posições das partículas.

Observação. Definindo a função $H: \mathcal{H} \rightarrow \mathcal{K}$ sendo $\mathcal{K}$ o espaço de definição do processo pontual $\Psi_{0}$, conseguimos $\Psi_{0}=H(\Pi()$.$) . Mas como o processo \Pi($.) é uma função do processo pontual $\mathbb{M}$, teremos que $\Psi_{0}=\tilde{H}(\mathbb{M})$, onde a função $\tilde{H}$ é a função composta 
$H(\Phi()$.$) . Em palavras, dado o processo \left\{\left(x_{n}, \xi_{n, m}\right), m, n \in Z\right\}$ conseguimos as trajetórias $\left\{\pi_{n}(t), n \in \mathbb{Z}\right\}$ e dadas as trajetórias obtemos $\left\{\sigma_{n}, n \in \mathbb{Z}\right\}$. Portanto, dado o processo $\left\{\left(x_{n}, \xi_{n, m}\right), n, m \in \mathbb{Z}\right\}$ conseguimos o processo $\left\{\left(x_{n}, \sigma_{n}\right), n \in \mathbb{Z}\right\}$.

Dado $\Psi_{0}$, definimos a fila associada usando a construção do processo workload $\{W(t), t \in$ $\mathbb{R}\}$ feito na seção anterior.

Definimos $W($.$) no intervalo (-\infty, \infty)$. O cliente $C_{0}$ chega à fila no instante $x_{0}=0$ quando a fila está vazia. O primeiro salto de $W($.$) é no ponto 0$ e o valor do salto é $W(0)=\sigma_{0}$. Sobre o intervalo $\left[0, x_{1}\right]$ o processo é de definido por

$$
W(t)= \begin{cases}0, & \text { se }-\infty<t<0 \\ {\left[\sigma_{0}-t\right]_{+}} & \text {se } 0<t<x_{1} \\ {\left[\sigma_{0}-\tau_{0}\right]_{+}+\sigma_{1}} & \text { se } t=x_{1}\end{cases}
$$

Onde $[a]_{+}:=\max \{0, a\}, a \in \mathbb{R}$.

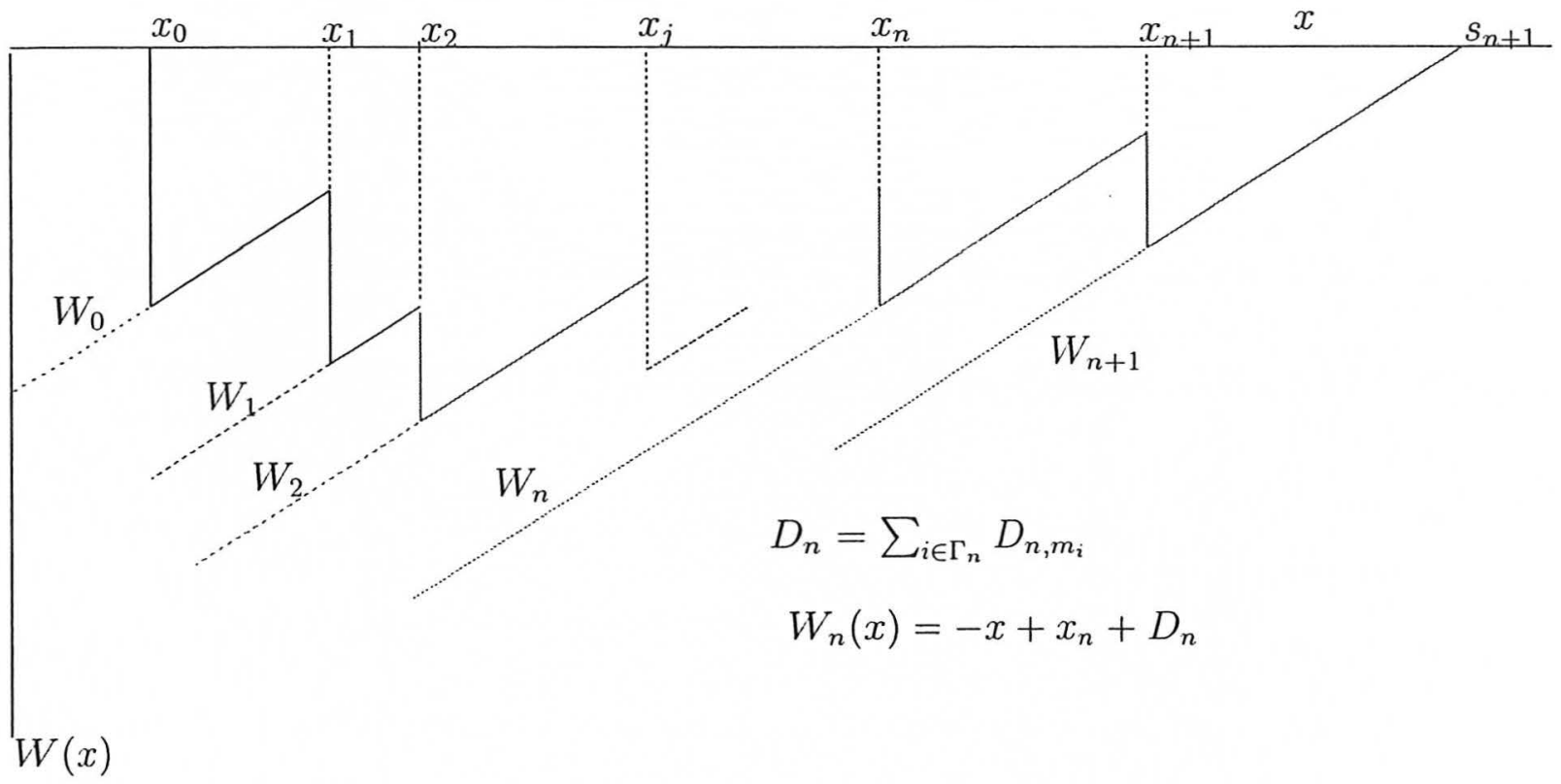

Figura 5.8: Fila Associada ao Modelo Pontual.

Se construimos $W($.$) sobre \left[0, x_{1}\right]$ podemos definir o processo $W($.$) sobre o intervalo$ $\left[x_{n}, x_{n+1}\right)$ para todo $n>1$.

$$
W(t)= \begin{cases}{\left[W_{n}\left(x_{n}\right)-\left(t-x_{n}\right)\right]_{+}} & \text {se } x_{n}<t<x_{n+1} \\ W_{n}\left(x_{n+1}-0\right)+\sigma_{n+1} & \text { se } t=x_{n+1}\end{cases}
$$


Onde $\sigma_{n+1}$ é o tempo de serviço do cliente $C_{n+1}$, definido pela equação 5.31 e pelo teorema 3. A magnitude de $\sigma_{n}$ pode-se obter como

$$
\sigma_{n+1}=W_{n+1}\left(x_{n+1}\right)-W_{n}\left(x_{n+1}\right) ; n \geq 0
$$

$W_{n}\left(\right.$.) é a função inversa da prolongação da trajetória $\pi_{n}(t)$ para $t>T_{n, m_{j}}$ da partícula $C_{n}$. Onde $m_{j}$ é o último ponto de bloqueio da partícula $C_{n}$. Da equação da trajetória da partícula sabemos que $\pi_{n}(t)$ para $t>T_{n, m_{j}}$ é

$$
\pi_{n}(t)=-t+x_{n}+D_{n} ; t>T_{n, m_{j}}
$$

Consideremos a prolongação desta trajetória desde o ponto $t=T_{n, m_{j}}$ até sua interseç̧ão com a reta $t=0$. Definamos a variável $t$ como a função $t=W_{n}(x)$, portanto, obtemos

$$
W_{n}(x)=-x+x_{n}+D_{n} ; x \in\left(x_{n}, s_{n}\right)
$$

Onde $s_{n}=x_{n}+D_{n}$ é definido como instante de saída do cliente $C_{n}$ da fila associada.

Definamos o tempo de espera $w_{n}:=W\left(x_{n}-0\right)$ como o tempo que se passa entre a entrada de cliente $C_{n}$ na fila e o momento em que começa seu serviço. No modelo de trânsito de partículas, $W_{n}\left(x_{n}\right)=D_{n}$, é o atraso total da $n$-ésima partícula. Para $w_{n}$ podese obter a relação de recorrência idêntica à relação de Lindley, $w_{n+1}=\left[w_{n}+\sigma_{n}-\tau_{n}\right]_{+}$, para $n \geq 0$, com $w_{0}=0$.

\subsubsection{Teoremas e Relações}

Discutiremos o teorema de estabilidade na fila associada ao modelo pontual.

Seja $\Psi=\left\{\left(r_{n+1}-r_{n}, \sigma_{n}\right), n \in \mathbb{Z}\right\}$ uma seqüência de variáveis iid independentes entre si tal que

$$
\ldots . r_{-2}<r_{-1} \leq 0<r_{0}<r_{1}<r_{2} \ldots
$$

Onde $\left\{\tau_{n}^{*}:=r_{n+1}-r_{n}, n \in \mathbb{Z}\right\}$ tempo entre chegadas do processo $\Psi$ é uma seqüência iid de média $1 / \lambda$. Consideremos a fila associada definida pelo processo $\Psi_{0}=\left\{\left(\tau_{n}, \sigma_{n}\right) ; n \in \mathbb{Z}\right\}$. As seqüências de variáveis são iid independentes entre si. $\left\{\tau_{n}, n \in \mathbb{Z}\right\}$ é o tempo entre chegadas e tem distribuição exponencial com média $1 / \lambda$. Clientes chegam ao sistema nos instantes de tempo $\left\{x_{n} ; n \in \mathbb{Z}\right\}$ tal que

$$
\ldots .<x_{-2}<x_{-1}<x_{0}=0<x_{1}<x_{2}<\ldots .
$$

Clientes entram na fila com seu tempo de serviço igual a $\left\{\sigma_{n} ; n \in \mathbb{Z}\right\}$ definido pelo teorema 1. O tempo de espera $w_{n+1}$ do cliente $C_{n+1}$ é expresso pela relação de Lindley.

$$
w_{n+1}= \begin{cases}w_{n}+\sigma_{n}-\tau_{n} & \text { se } w_{n}+\sigma_{n}<\tau_{n} \\ 0 & \text { se } w_{n}+\sigma_{n} \leq \tau_{n}\end{cases}
$$

Para todo tempo $n \in \mathbb{Z}$. Definindo $\eta_{n}:=\sigma_{n}-\tau_{n}$ temos 


$$
w_{n+1}=\left[w_{n}+\eta_{n}\right]_{+}=: f\left(w_{n}, \eta_{n}\right), n \in \mathbb{Z}
$$

Teorema 6 [Estabilidade da fila]. Consideremos $\Psi_{0}$ uma versão sincronizada (versão de Palm) do processo $\Psi$ que é estacionário e ergódico. Se $\mathbb{E} \eta_{n}=\mathbb{E} \sigma_{n}-\mathbb{E} \tau_{n}<0$. Então, o processo $\left\{w_{n}, n \in \mathbb{Z}\right\}$ é estável. Isto é, a distribuição de $w_{n}$ quando $n \rightarrow \infty$ converge para uma variável aleatória finita $\theta$, independentemente da condição inicial. Além disso, existe um número infinito de índices negativos $n$ e um infinito número infinito de índices positivos $n$ tais que $w_{n}=0$

A prova do teorema será baseada nos seguintes lemas

Lema 1 [Limite de $w_{n}$ ]. Dadas as condições do teorema 6 e dado o processo $\left\{w_{n}, n \in \mathbb{Z}\right\}$. Então, $w_{n}$ converge para uma variável aleatória $\theta$ que pode ser infinita.

\section{Prova.}

Dado $\Psi_{0}=\left\{\left(\tau_{n}, \sigma_{n}\right), n \in \mathbb{Z}\right\}$. Para cada $m \leq n$ podemos resolver a equação 5.45 para $w_{n}$ em termos de $w_{m}$ e $\Psi_{\mathbf{0}}$. Escrevemos a solução como

$$
w_{n}=F\left(m, n ; w_{m}, \Psi_{0}\right)
$$

Deste modo $F\left(m, n ; x, \Psi_{\mathbf{0}}\right)$ é o valor de $w_{n}$ dado que $w_{m}=x$ e o processo de entrada é $\Psi_{0}$. Observemos que a equação 5.45 está definida pela função $f$ que é a função máximo o que implica que $F\left(n, m ; x, \Psi_{0}\right)$ é não decrescente e contínuo à esquerda em $x \in[0, \infty]$ para todo $\Psi_{0}$ e para todo $m \leq n$. Estas propriedades serão conservadas na composição de função $f$.

Temos que provar que: Dado $w_{0}=0$ e considerando $\Psi_{0}$ (evento estacionário) versão do Palm do processo estacionário $\Psi$. Então, a distribuição de $w_{n}$ quando $n \rightarrow \infty$ converge para a variável $\theta$ que pode ser infinita. Para provar isto, observemos que

$$
F\left(m-1,0 ; 0, \Psi_{0}\right)=F\left(m, 0 ; f\left(0, \eta_{m-1}\right), \Psi_{0}\right) \geq F\left(m, 0 ; 0, \Psi_{0}\right), \quad m \leq 0
$$

A primeira igualdade mostra que se começamos com $w_{m-1}=0$ teremos que $w_{m}=$ $f\left(0, \eta_{m-1}\right)$ porque $w_{m}=f\left(w_{m-1}, \eta_{m-1}\right)$. A desigualdade segue do fato que $F\left(m, 0 ; x, \Psi_{0}\right)$ é não decrescente em $x$. A desigualdade $F\left(m-1,0 ; 0, \Psi_{0}\right) \geq F\left(m, 0 ; 0, \Psi_{0}\right)$ mostra que $w_{0}$ começando com $w_{m-1}=0$ é maior que $w_{0}$ começando em $w_{m}=0$ o que implica que $F\left(m, n ; 0, \Psi_{0}\right)$ é não decrescente em $m$ e portanto converge para a variável aleatória $\theta$ quando $m \downarrow-\infty$. Onde $\theta$ é definido por $\theta:=\lim _{m \rightarrow-\infty} F\left(m, 0 ; 0, \Psi_{0}\right)$.

Como $\Psi_{0}$ é evento estacionário teremos que

$$
F\left(-n, 0 ; 0, \Psi_{0}\right) \text { e } w_{n}=F\left(0, n ; 0, \Psi_{0}\right)
$$

tem a mesma distribuição para $n>0$. Esta última igualdade mostra que a distribuição de $w_{n}$ converge para esse mesmo $\theta$ quando $n \rightarrow \infty$. 
Lema 2 [Distribuição estacionária]. Dadas as condições do teorema 6 , então, o processo $\left\{w_{n}, n \in \mathbb{Z}\right\}$ é estacionário.

\section{Prova.}

Provaremos que se escolhemos $w_{0}=\theta$ então o processo $\left\{w_{n}, n \in \mathbb{Z}\right\}$ é estacionário. Da definição de $F$ dada na equação 5.46 segue que

$$
F\left(m, n ; 0, \Psi_{0}\right)=F\left(0, n ; F\left(m, 0 ; 0, \Psi_{0}\right) \Psi_{0}\right) \text { para } m<0 \leq n
$$

Esta igualdade mostra que o valor $w_{n}$ começando de $w_{m}=0$ é o mesmo que o valor de $w_{n}$ começando de $w_{0}=F\left(m, 0 ; 0, \Psi_{0}\right)$, onde $F\left(m, 0 ; 0, \Psi_{0}\right)$ é a condição inicial .

Tomando limite quando $m \downarrow-\infty$ e usando a continuidade a esquerda de $F$ e definido $\theta\left(n, \Psi_{\mathbf{0}}\right):=\lim _{m \rightarrow-\infty} F\left(m, n ; 0, \Psi_{\mathbf{0}}\right)$ obtemos

$$
\theta\left(n, \Psi_{\mathbf{0}}\right)=F\left(0, n ; \theta, \Psi_{\mathbf{0}}\right), \quad n \geq 0
$$

Disto,

$$
\left\{\theta\left(n+k, \Psi_{0}\right), 1 \leq k \leq L\right\}=\left\{F\left(0, n+k ; \theta, \Psi_{0}\right), 1 \leq k \leq L\right\}
$$

Agora,

$$
\left\{\theta\left(n+k, \Psi_{0}\right), 1 \leq k \leq L\right\}=\left\{\theta\left(k, \Psi_{0}+n\right), 1 \leq k \leq L\right\}
$$

Onde $\Psi_{0}+n:=\left\{\left(x_{m}-x_{n}, \sigma_{m}\right), m \in \mathbb{Z}\right\}$. O sinal nesta definição pode ser confuso. Lembremos que $\Psi_{0}+n$ é o processo $\Psi_{0}$ observado por mover a origem ao ponto $x_{n}$. Desde que $\Psi_{0}$ é evento estacionário, segue que a distribuição do lado esquerdo da equação 5.52 é estacionária (isto é, independente de $n \geq 0$ ). A equação 5.52 mostra que este processo estacionário é precisamente o processo $\left\{w_{n}, n \geq 0\right\}$ quando $w_{0}=\theta=\theta\left(0, \Psi_{0}\right)$.

Lema 3 [ $\theta$ finito quase certamente]. Dadas as condições do teorema 6 , então, o processo $\left\{w_{n}, n \in \mathbb{Z}\right\}$ é finito com probabilidade 1

\section{Prova.}

Usando a forma explícita de $f(x, y)=[x+y]_{+}$para calcular $w_{0}$ dada a condição inicial $w_{-n}=0$, para $n \geq 1$. Por indução encontramos

$$
F\left(-n, 0 ; 0, \Psi_{0}\right)=\max \left\{0, \eta_{-1}, \eta_{-1}+\eta_{-2}, \ldots, \eta_{-1}+\ldots+\eta_{-n}\right\}
$$

para $n \geq 1$. Portanto,

$$
\theta=\left[\sup _{n \geq 1} \sum_{m=1}^{n} \eta_{-m}\right]_{+}
$$

Como $\Psi$ é estacionário e ergódico, então $\Psi_{0}$ é também ergódico e evento estacionário, portanto concluimos que com probabilidade 1 


$$
\lim _{n \rightarrow \infty} \frac{1}{n} \sum_{m=1}^{n} \eta_{-m}=\mathbb{E} \eta_{0}
$$

Dada a condição que $\mathbb{E} \eta_{0}<0$, então, com probabilidade 1 cumpre-se

$$
\lim _{n \rightarrow \infty} \sum_{m=1}^{n} \eta_{-m}=-\infty \text { se } \mathbb{E} \eta_{0}<0
$$

Isto mostra que $\mathbb{P}(\theta<\infty)=1$. Porque a seqüência que converge para $-\infty$ deve de ter um supremo finito.

Lema 4 [Ciclos da fila]. Dadas as condições do teorema 6, então, os pontos onde chegam clientes quando a fila está vazia são pontos de renovação.

\section{Prova.}

Definimos $M_{i}=\min \left\{m \leq i: D_{i, m}>0\right\} . \quad M_{i}$ é a posição aleatória da partícula mais distante da partícula $C_{i}$ tal que ocasiona um atraso positivo. $M_{i}$ pode ser $-\infty$, neste caso a partícula $C_{i}$ será atrasada infinitas vezes.

Consideremos o evento $\left\{M_{i}=i\right\}$, isto é, o evento o atraso inicial da partícula $C_{i}$. Da dinâmica das partículas vemos que este evento tem probabilidade positiva e a função indicadora do evento é função que depende só do passado até o instante $i$. Portanto,

$$
I\left(M_{i}=i\right)=h\left[\left(\tau_{j}, \sigma_{j}\right), j \leq i\right]
$$

Usando a ergodicidade de $\Psi_{\mathbf{0}}$. Pelo teorema ergódico encontramos

$$
\lim _{n \rightarrow \infty} \frac{1}{n} \sum_{i=1}^{n-1} I\left(M_{i}=i\right)=\mathbb{E} I\left[M_{i}-i\right]=\mathbb{P}\left(M_{i}=i\right)>0
$$

Isto implica que existe um número infinito de índices negativos $n$ e um infinito número infinito de índices positivos $n$ tais que $w_{n}=0$

\section{Prova de teorema 6.}

Para provar a estabilidade da fila tendo em vista os lemas 1, 2 e 3 é suficiente mostrar a eventual independência da condição inicial. Isto pode ser feito provando que o sistema dado pela equação 5.46 tem perda de memória, quer dizer, que para todo $x>0$ existe um $n\left(x, \Psi_{0}\right)$ finito tal que

$$
F\left(0, n ; x, \Psi_{0}\right)=F\left(0, n ; 0, \Psi_{0}\right) \text { para } n \geq n\left(x, \Psi_{0}\right)
$$

Deste modo, depois de um número finito de chegadas, a seqüência de tempos de espera é a mesma quando as condições iniciais são $w_{0}=x$ ou $w_{0}=0$. Disto segue que $\left\{w_{n}, n \in \mathbb{Z}\right\}$ é independente de $w_{0}$. 
Para mostrar 5.59, é suficiente mostrar que $F\left(0, n ; x, \Psi_{0}\right)=0$ para algum $n$ finito tal que $n>n\left(x, \Psi_{0}\right)$. Na verdade temos que

$$
0 \leq F\left(0, n ; 0, \Psi_{0}\right) \leq F\left(0, n ; x, \Psi_{0}\right) \text { para todo } n \geq 0
$$

Escolhamos $w_{0}=x$ e assumindo que $w_{n}>0$ para todo $n \geq 0$. Então, a equação 5.46 nos dá

$$
w_{n+1}=w_{n}+\eta_{n}, n \geq 0
$$

então,

$$
w_{n}=x+\sum_{m=0}^{n-1} \eta_{m}, n>0
$$

Isto é claramente impossível porque $\sum_{m=0}^{n-1} \eta_{m} \rightarrow-\infty$ quando $n \rightarrow \infty$. Portanto, $w_{n}$ deve ser eventualmente 0 , quer dizer que existe um $n\left(x, \Psi_{0}\right)$.tal que $w_{n\left(x, \Psi_{0}\right)}=0$

\section{Processo de saídas.}

Podemos construir o processo das saídas do sistema calculando o instante de saída de cada cliente. Seja $s_{n}$ o instante de saída do $n$-ésimo cliente. Dado que o $n$-ésimo cliente entrou no instante $x_{n}$, espero na fila $w_{n}$ e logo demorou o tempo $\sigma_{n}$ no atendimento. Seu tempo de saída será $s_{n}=x_{n}+w_{n}+\sigma_{n}$, para $n \in \mathbb{Z}$.

\section{Prova alternativa da estacionariedade do processo $w_{n}$ na fila associada.}

Para provar a estacionariedade da fila associada usaremos o conceito de ciclo estacionário introduzido por Thorisson[29] (Capítulo 8). Temos definido por ciclo da fila a distância entre as entradas de dois clientes que encontraram a fila vazia. Supondo que o cliente $C_{0}$ encontra a fila vazia. O período ocupado que começa no zero se define como o intervalo aleatório

$$
\left[0, T_{j}\right] \text { onde } j:=\min \left\{m>0: W\left(s_{m}\right)=0\right\}
$$

O intervalo de tempo desde a última saída até a próxima chegada denominamos de período desocupado. Definimos este período como o intervalo

$$
\left.\left[T_{j}, S_{k}\right] \text { onde } k:=\min \left\{m>0: W\left(x_{m}-0\right)=0\right)\right\}
$$

Então, temos que $\left[0, S_{k}\right]$ é o ciclo que é a soma de um período ocupado mais um período desocupado adjacente.

Proposicão 1 [Ciclo estacionário]. Dado o processo pontual marcado $\Psi_{0}=$ $\left\{\left(\tau_{n}, \sigma_{n}\right) ; n \in \mathbb{Z}\right\}$ com seqüências independentes entre si. Os tempos entre chegadas são dados pela seqüência $\left\{\tau_{n}=x_{n+1}-x_{n}, n \in \mathbb{Z}\right\}$ que são iid exponenciais de média $1 / \lambda$. $\left\{\sigma_{n}, n \in \mathbb{N}\right\}$ é uma seqüência iid com distribuição exponencial de média 1 que define os 
últimos tempos de espera para que a $n$-ésima partícula mude sua velocidade de 0 a -1 para sempre. Dado $T_{j}$ definido por

$$
T_{j}=\left[0, x_{j}\right], \text { tal que } j=\min \left\{m>0: W\left(s_{m}\right)=0\right\}
$$

Onde $s_{m}$ é a saída do cliente $C_{m}$. Se $\mathbb{E} \sigma_{n}<\mathbb{E} \tau_{n}$, então, $\mathbb{E} T_{j}<\infty$, para todo $j \in \mathbb{Z}$.

\section{Prova.}

Na fila associada consideremos o período ocupado que começa quando o cliente $C_{0}$ entra na fila vazia e que termina com a saída do cliente $C_{j}$ deixando a fila também vazia. Nestas condições estabeleceremos a seguinte relação entre os os tempos de serviço $\sigma_{n}$ e os tempos entre chegadas $\tau_{n}$.

$$
\sum_{i=0}^{j} \sigma_{i}=\sum_{i=0}^{j-1} \tau_{i}+W\left(x_{j}\right)
$$

Pondo em evidência $W\left(x_{j}\right)$, temos

$$
W\left(x_{j}\right)=\sigma_{0}+\sum_{i=1}^{j}\left(\sigma_{i}-\tau_{i+1}\right)
$$

Da condição $\mathbb{E} \sigma_{n}<\mathbb{E} \tau_{n}$ temos que $\mathbb{E}\left(\sigma_{n}-\tau_{n}\right)<0$. Usando o teorema ergódico

$$
\frac{1}{n}\left\{\sum_{k=1}^{n}\left(\sigma_{k}-\tau_{k}\right)\right\} \rightarrow \mathbb{E}\left(\sigma_{1}-\tau_{1}\right)
$$

Teremos que $\sum_{k=1}^{\infty}\left(\sigma_{k}-\tau_{k}\right) \rightarrow-\infty$.

Como $W() \geq$.0 , existe um $m<\infty$ tal que $W\left(x_{m}\right)=0$. Então, a esperança do período ocupado é finito quase certamente. O período desocupado tem distribuição exponencial de média $1 / \lambda$ e também tem esperança finita. Isto prova o teorema.

Teorema 7 [O processo $w_{n}$ é estacionário]. Dado o processo pontual marcado $\Psi_{0}=\left\{\left(\tau_{n}, \sigma_{n}\right) ; n \in \mathbb{Z}\right\}$ com seqüências independentes entre si. Os tempos entre chegadas são dados pela seqüência $\left\{\tau_{n}=x_{n+1}-x_{n}, n \in \mathbb{Z}\right\}$ que são iid exponenciais de média $1 / \lambda$. $\left\{\sigma_{n}, n \in \mathbb{N}\right\}$ é uma seqüência iid com distribuição exponencial de média 1 que define os últimos tempos de espera para que a $n$-ésima partícula mude sua velocidade de 0 a -1 para sempre. Se $\mathbb{E} \sigma_{n}<\mathbb{E} \tau_{n}$, então, o processo $w_{n}$ é estacionário.

\section{Prova.}

Se provamos que o processo $w_{n}$ é ciclo estacionário, então, este será estacionário por usar o resultado de Thorisson[29], theorem 4.1, capítulo 8.

O processo $w_{n}$ é ciclo estacionário ver proposição 1 . 


\subsection{Prova dos Resultados}

Enunciaremos de novo os teoremas.

Teorema 1. Dado o processo pontual marcado $\mathbb{M}$. Se $\lambda<1$.

a. Então, $\mathbb{P}\left(M_{n}(\mathbb{M})<\infty\right)=1$ e o tempo de espera para que a partícula passe ao estado livre é dado por $T_{M_{n}}=D_{n}+\left(x_{n}-x_{M_{n}}\right)$. Onde $D_{n}=\sum_{m} D_{n, m}$ é a soma do todos os atrasos da partícula $C_{n}$ e $M_{n}(\mathbb{M})$ é definido por

$$
M_{n}(\mathbb{M})=\min \left\{m \leq n: D_{n, m}=t_{n, m}^{+}-t_{n, m}^{-}>0\right\}
$$

b. Além disso,

$$
\lim _{t \rightarrow \infty}\left(\pi_{n}(t)-\pi_{n-1}(t)\right)=\tilde{s}_{n}-\tilde{s}_{n-1} \text { quase certamente }
$$

Onde $\tilde{s}_{n}$ é o tempo de saída do $n$-ésimo cliente de uma fila estacionária $M / M / 1 / \infty$ associada ao sistema.

\section{Prova do teorema 1.}

A prova do teorema é baseada na representação do modelo de trânsito como uma fila com um único servidor.

Consideremos o processo workload $\{W(t), t \in \mathbb{R}\}$ definido para a fila associada. A partícula $C_{n}$ para cada $n$, tem associado seu conjunto de pontos de atraso, $\Gamma_{n}=\left\{m_{k}, k \in\right.$ $\mathbb{N}$ \}. Esta seqüência pode ser finita ou infinita, se for infinita significa que a partícula é atrasada infinitas vezes. Da dinâmica das partículas e da definição do processo $W($.$) para$ cada partícula $C_{n}, n \in \mathbb{Z}$. $W\left(x_{n}\right)$ é soma de todos os seus atrasos, isto é,

$$
W\left(x_{n}\right):=\sum_{k} D_{n, m_{k}} \text { e } w_{n}:=W\left(x_{n}-0\right)
$$

é o tempo total que a partícula $C_{n}$ permanece bloqueada.

Prova a. Assumindo que a partícula da origem está livre desde o início. Consideremos a partícula $C_{n}$ e sua posição $x_{n} \operatorname{com} n$ suficientemente grande. Como $\lambda<1$, então $w_{n}$ é exponencial.

Supondo que $\mathbb{P}\left(M_{i}(\mathbb{M})=\infty\right)=1$, isto implica que temos associado à partícula $C_{n}$ uma seqüência infinita $\Gamma_{n}=\left\{m_{k}, k \in \mathbb{N}\right\}$ de pontos de atraso. O workload será $W\left(x_{n}\right):=\sum_{k} D_{n, m_{k}}$. Pelo teorema dos grande números, temos que está soma converge para infinito, o que é impossível, porque o workload é finito com probabilidade 1. Portanto, $M_{i}$ é finito com probabilidade 1 .

Prova b. Assumindo que a partícula na origem está livre desde o início. Consideremos $C_{n}$ e $C_{n+1}$, com $n$ suficientemente grande. Como $\lambda<1$ cumpre-se o teorema 6 e para $C_{n}$ e $C_{n+1}$ as saídas serão os pontos $\tilde{s}_{n}$ e $\tilde{s}_{n+1}$ que pela estabilidade da fila serão 


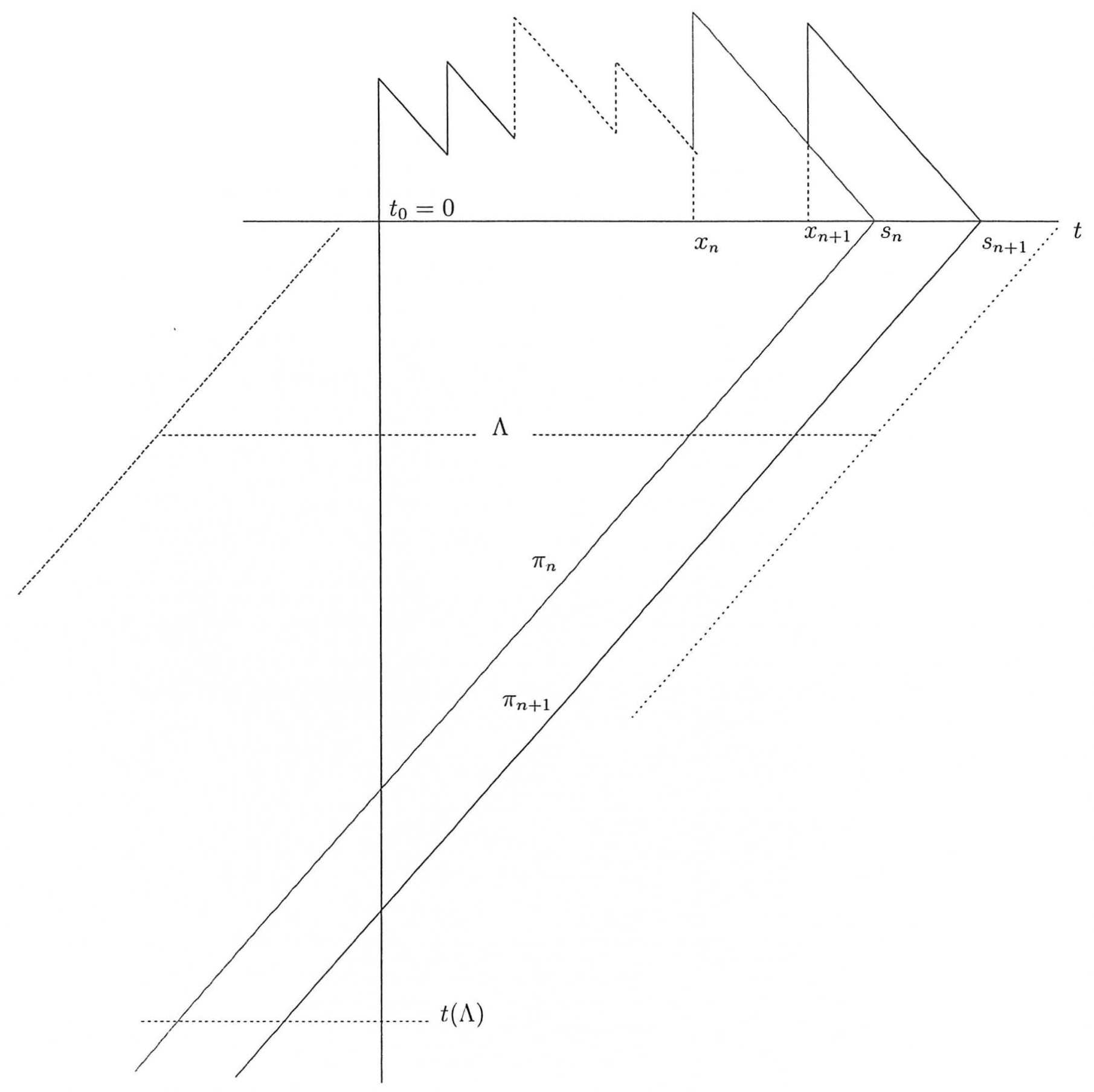

Figura 5.9: Partícula $C_{n}$ e $C_{n+1}$ para o teorema 1. 
finitos quase certamente. Estas partículas cruzam o eixo dos tempos nos pontos $\tilde{s}_{n}$ e $\tilde{s}_{n+1}$. Então, para todo $t>s_{n+1}$, graficamente pode-se comprovar que $\pi_{n+1}-\pi_{n}=\tilde{s}_{n+1}-\tilde{s}_{n}$. Portanto, no limite quando $t \rightarrow \infty$ também se cumprirá.

Observação. Na figura 5.9 que ilustra a prova do teorema, temos definido o intervalo $\Lambda$. Este intervalo move-se à velocidade constante -1 junto com as partículas. O teorema 1 será satisfeito se existe um $T:=T(\Lambda)$ tal que para todo $t>T(\Lambda)$ teremos que: As partículas do processo $\left\{\pi_{n}(t), n \in \mathbb{Z}\right\}$ que pertencem ao intervalo $\Lambda-t$ corresponde às saídas da fila associada que pertencem ao intervalo $\Lambda$. Aqui definimos $\Lambda-t=\{x-t, x \in$ $\Lambda\}$.

Da figura 5.9 basta definir um intervalo $\Lambda$ tal que a cota superior do intervalo esteja mais à direita que a saída da partícula $C_{n+1}$, com isto, elegimos um $T(\Lambda)$ que seja maior que $\tilde{s}_{n+1}$, então, para todo $t>T$ cumpre-se o teorema 1 .

Teorema 2. Dado o processo pontual marcado M. Se $\lambda<1$.

a. Existe um $T=T(\mathbb{M})<\infty$ aleatório, tal que, se $t>T$, todas as partículas que passam pela origem depois de $T$ são livres.

b. O processo pontual no eixo temporal $X=0$ é Poisson a partir desse $T(\mathbb{M})$

\section{Prova do teorema 2}

Prova a. Assumindo que a partícula é livre desde o início. Como $\lambda<1$ a fila associada ao modelo pontual está em equilíbrio e nosso sistema de partículas se transformou num conjunto infinito enumerável de ciclos de tamanho finito. Consideremos o ciclo de maior tamanho possível que começa na origem. Definimos o tempo aleatório $T(\mathbb{M})$ como $T(\mathbb{M}):=\max _{k \in N}\left\{S_{k}\right\}$ onde $S_{k}$ é o tamanho do ciclo. Com isto, o próximo ciclo de partículas começa com uma partícula livre, desde o início, que cruzará a origem depois do tempo $T$, estando livre por ser o primeiro do novo ciclo. A partir desta partícula, todas as demais também cruzarão a origem em estado livre, porque devido à estabilidade da fila, estas sairão da fila depois de tempos finitos e quando passarem pela origem, estarão em estado livre.

Prova b. Se $\lambda<1$, a fila associada está em equilíbrio. Definimos $T(\mathbb{M})$ como na parte a, então, as saídas $\left\{\tilde{s}_{n} ; \tilde{s}_{n}>T\right\}$ da fila formam um processo de Poisson. As partículas do próximo ciclo à direita cruzarão a origem depois de tempo $T$. Estas partículas passarão pela origem em fluxo livre a partir deste tempo $T$ e pelo teorema 1 estarão separados por uma distância que converge à distância entre as saídas da fila. Como as saídas da fila forma um processo de Poisson, então, as distâncias entre as partículas no eixo $X=0$ formará também um processo de Poisson (Ver figura 5.10).

Observação. Da figura 5.10 vemos que basta eleger um intervalo $\Lambda$ que inicie no 


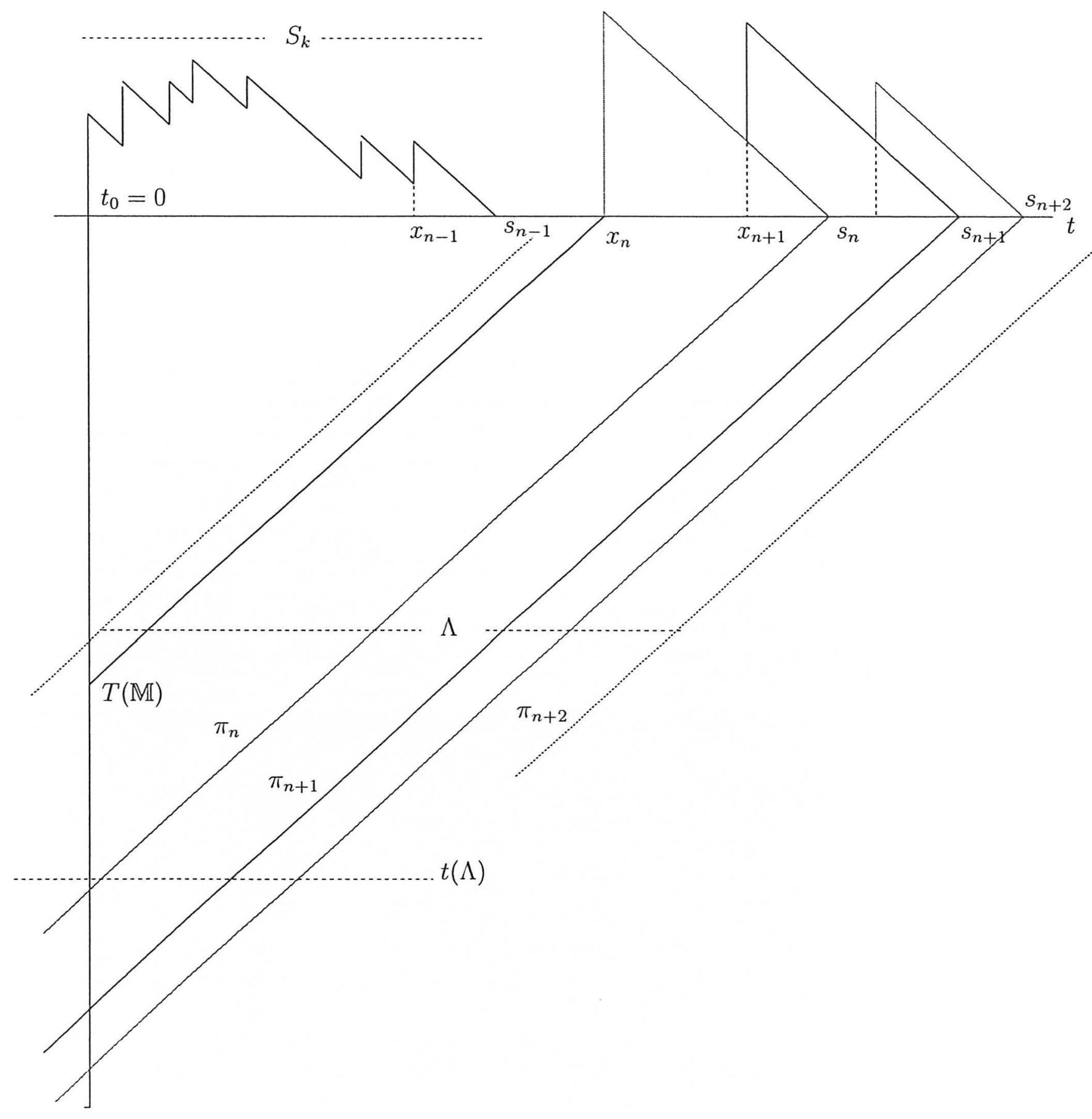

Figura 5.10: Ciclo máximo $S_{k}$ que começa na origem para o teorema 2. As partículas do ciclo consecutivo formam no eixo $X=0 \mathrm{um}$ processo de Poisson. 
primeiro período desocupado e que cubra qualquer ciclo completo começando do segundo. Em particular escolhemos $\Lambda$ que começa na posição da primeira partícula do segundo ciclo, então, definimos $T(\Lambda):=T(\mathbb{M})$. Logo, para todo $t>T$ cumpre-se o teorema 2 . 


\section{Referências Bibliográficas}

[1] O. Angel, A. Holroyd, J. Martin, The Jammed Phase of the Biham-Middleton-Levine traffic model. Preprint arXiv:math.PR/0504001.

[2] S. Asmussen, Applied Probability and Queues. Wiley Series In Probability and Mathematical Statistics, 1987.

[3] F, Baccelli, P. Brémaud, Element of Queueing Theory. First ed, Springer, 1994.

[4] R. Barlovic, L. Santen, A. Schadscheneider, M. Schreckenberg, Metastable States in Cellular Automata for Traffic Flow. The Eur. Phys. J. B 5, 793-800, (1998).

[5] V. Belitsky, P. Ferrari, Ballistic Annihilation and Deterministic Surface Growth, J. Stat. Physics. vol 80 3/4, 517- 543, (1995).

[6] V. Belitsky, P. Ferrari, Invariant Measures and Convergence for Cellular Automaton 184 and Related Processes, J. Stat Phys. vol 118, 3/4, 589-623, (2005)

[7] V. Belitsky, J. Krug, E. Neves, G. Schutz, A Cellular Automaton Model for two-laneTraffic, J. Stat. Phys, 103, 945-971, (2001)

[8] M. Blank, Ergodic Properties of a Simple Deterministic Traffic Flow Model, J. Stat. Phys. Vol 111, 903-930, (2003)

[9] M. Blank, Hysteresis Phenomenon in Deterministic Traffic Flow. Preprint arXiv:math.DS/0408240.

[10] N. Boccara, H. Fuks, Critical Behavior of a Cellular Automaton Highway Traffic Model. Preprit arXiv: cond-mat/9911039.

[11] D. Chowdhury, 1. Santen, A. Schadschneider, Statistical Physics of Vehicular Traffic and Some Related Systems. Phys. Rep. 329, 199-329, (2000)

[12] Y. Elskens, H. L. Frisch, Annihilation Kinetics in the One-dimensional Ideal Gas, Physical Review A, Vol.31, No 6, 3812-3816, (1985).

[13] W. Feller, Introducción a la Teoria de Probabilidades y sus Aplicaciones. Vol 1. Editorial Limusa, Mexico, 1975. Tradução da terceira edição publicada em Inglês por John Wiley and Sons, 1968. 
[14] H. Fuks, Exact Result for Deterministic Cellular Automaton Traffic Models, Phys Rev E 60, 197-202, (1999).

[15] H. Fuks, N. Boccara, Convergence to Equilibrium in a Class of Interacting Particle Systems Evolving in Discrete Time, Phys Rev E 64, 016117, (2001).

[16] L. Gray, D. Griffeath, The Ergodic Theory of Traffic Jams, J. Stat Phys, Vol 105, Nos $3 / 4$ (2001).

[17] H. Greenberg, Oper. Res. 7, 79, (1959)

[18] D. Helbing, Traffic and related self-driven many-partivle systems, Reviews of Modern Physics, Vol 73, 1067-1141, (2001).

[19] J. Krug, H. Spohn, Universality Classes for Deterministic Surface Growth. Physical Review A, Vol. 38, 4271-4283, (1988)

[20] T. Liggett, Interacting Particles Systems. Springer-Verlag, 1985.

[21] R. Loynes, The Stability of teh Queue with no Independent Interarraival and Service Time. Proc. Camb. Philos. Soc, 58,497-520, (1962).

[22] K. Nagel; M. Schreckenberg, A Cellular Automaton Model for Freeway Traffic, J. Physique I2:2221-2229 (1992).

[23] E. Pechersky, F. Castellares, Car Traffic and Queuing. Em preparação.

[24] N. Prabhu, Stochastic Storage Processes: Queues, Insurance Risk, Dams, and Data Cominication. Second edition, Springer, 1998.

[25] N. Prabhu, Queues and Inventories. Wiley, New York, 1965.

[26] A. Schadschneider, M. Schreckenberg, Traffic Flow Models with "slow-to-stars"Rules. Ann. Physik 6, 541-555, 1997.

[27] K. Sigman, Stationary Marked Point Processes: An Intuitive Approach. Chapman and Hall, 1995.

[28] A. Shiryaev, Probability. Second edition, Springer, 1984.

[29] H. Thorisson, Coupling, Stationarity and Regeneration. Springer-Verlag New York, 2000 .

[30] H. Yaguchi, Stationary Measures for an Exclusion Process on-dimensional Lattices with Infinitely Many Hopping Sites, Hiroshima. Math. J, 16, 449-474, (1986).

[31] S. Wolfram, Cellular Automata and Complexity. Collected Papers, Addison-Wesley Publishing Company, 1994. 